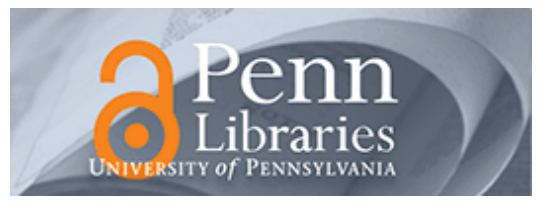

University of Pennsylvania ScholarlyCommons

7-5-2018

\title{
Vertical hopper compositions for preflexive and feedback- stabilized quadrupedal bounding, pacing, pronking, and trotting
}

Avik De

University of Pennsylvania, avik@seas.upenn.edu

Daniel E. Koditschek

University of Pennsylvania, kod@seas.upenn.edu

Follow this and additional works at: https://repository.upenn.edu/ese_papers

Part of the Electrical and Computer Engineering Commons, and the Systems Engineering Commons

\section{Recommended Citation}

Avik De and Daniel E. Koditschek, "Vertical hopper compositions for preflexive and feedback-stabilized quadrupedal bounding, pacing, pronking, and trotting", The International Journal of Robotics Research 37(7), 743-778. July 2018. http://dx.doi.org/10.1177/0278364918779874

This paper is posted at ScholarlyCommons. https://repository.upenn.edu/ese_papers/832

For more information, please contact repository@pobox.upenn.edu. 


\title{
Vertical hopper compositions for preflexive and feedback-stabilized quadrupedal bounding, pacing, pronking, and trotting
}

\author{
Abstract \\ This paper applies an extension of classical averaging methods to hybrid dynamical systems, thereby \\ achieving formally specified, physically effective and robust instances of all virtual bipedal gaits on a \\ quadrupedal robot. Gait specification takes the form of a three parameter family of coupling rules \\ mathematically shown to stabilize limit cycles in a low degree of freedom template: an abstracted pair of \\ vertical hoppers whose relative phase locking encodes the desired physical leg patterns. These coupling \\ rules produce the desired gaits when appropriately applied to the physical robot. The formal analysis \\ reveals a distinct set of morphological regimes determined by the distribution of the body's inertia within \\ which particular phase relationships are naturally locked with no need for feedback stabilization (or, if \\ undesired, must be countermanded by the appropriate feedback), and these regimes are shown \\ empirically to analogously govern the physical machine as well. In addition to the mathematical stability \\ analysis and data from physical experiments we summarize a number of extensive numerical studies that \\ explore the relationship between the simple template and its more complicated anchoring body models. \\ For more information: Kod*lab \\ Keywords \\ legged robots, dynamics, hybrid systems \\ Disciplines \\ Electrical and Computer Engineering | Engineering | Systems Engineering
}




\title{
Vertical hopper compositions for preflexive and feedback-stabilized quadrupedal bounding, pacing, pronking, and trotting
}

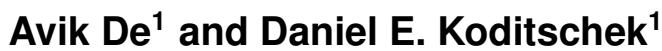

\begin{abstract}
This paper applies an extension of classical averaging methods to hybrid dynamical systems, thereby achieving formally specified, physically effective and robust instances of all virtual bipedal gaits on a quadrupedal robot. Gait specification takes the form of a three parameter family of coupling rules mathematically shown to stabilize limit cycles in a low degree of freedom template: an abstracted pair of vertical hoppers whose relative phase locking encodes the desired physical leg patterns. These coupling rules produce the desired gaits when appropriately applied to the physical robot. The formal analysis reveals a distinct set of morphological regimes determined by the distribution of the body's inertia within which particular phase relationships are naturally locked with no need for feedback stabilization (or, if undesired, must be countermanded by the appropriate feedback), and these regimes are shown empirically to analogously govern the physical machine as well. In addition to the mathematical stability analysis and data from physical experiments we summarize a number of extensive numerical studies that explore the relationship between the simple template and its more complicated anchoring body models.
\end{abstract}

\section{Keywords}

Legged Robots, Dynamics, Motion Control

\section{Introduction}

In this paper we apply a dynamical systems method for coupling hybrid oscillators De et al. (2018) to achieve formally specified, physically effective and robust instances of all virtual bipedal gaits on a quadrupedal robot. Gait specification takes the form of a three-parameter family ( $k_{a}, k_{p}$, and $k_{d}$ to be introduced in (8)-(10) ${ }^{1}$ ) of coupling rules used to entrain an otherwise independent pair of virtual vertical leg hopping controllers. The specification is formal in the sense that we give mathematical stability proofs for the entrained target limit cycle resulting from application of these coupling rules to a reduced degree of freedom template Full and Koditschek (1999) model for each combination of mechanical coupling and desired phase relation. The gaits are physically effective in the sense that appropriate projections of the robot's $6 \mathrm{DOF}$ (twelve dimensional) in-place hopping trajectories match closely those of the 2DOF (four dimensional) template when subject to the same coupling rules tuned to the appropriate parameter settings. The gaits are robust in the sense that they persist in the face of mechanical cross-talk arising from adjoining fore-aft excitation control terms to those commanding the coupled vertical hopping, yielding reliable bounding, pacing, pronking, and trotting behaviors in the physical machine. In many of the configurations we study, this robust gait stability can be formally attributed to natural stabilizing forces arising from intrinsic body morphology that have been termed preflexes in the biomechanics literature to distinguish them from feedback (reflex) endowed stability. For the reader's convenience, we include a definition here:

Definition 1. Preflex Brown and Loeb (2000). Stabilizing forces generated through the interaction of the body mechanics with contact forces, without any work output from actuators.

\subsection{Related work}

Biological inspiration Almost all biological quadrupeds exhibit one or more effectively bipedal gaits wherein

\footnotetext{
${ }^{1}$ Electrical and Systems Engineering, University of Pennsylvania, Philadelphia, PA, USA.
}

Corresponding author:

Avik De, Philadelphia, PA 19103

Email: avik@seas.upenn.edu 


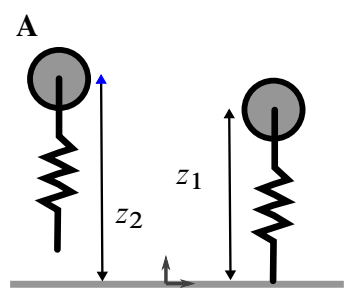

Independent hoppers

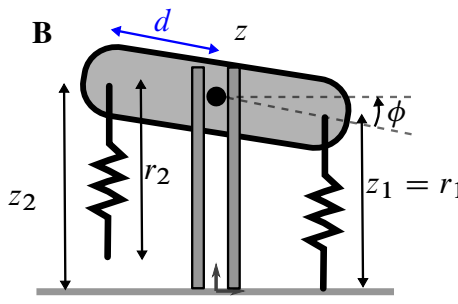

Slot hopper

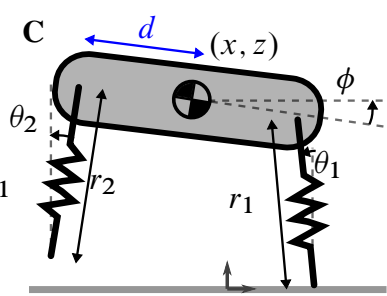

Sagittal plane biped

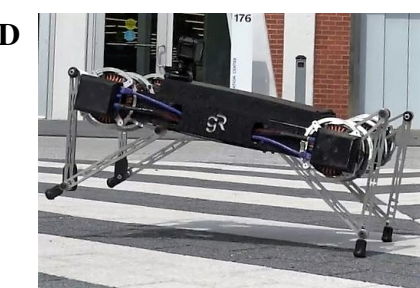

Minitaur experimental platform

Figure 1. Virtual biped models of increasing complexity. Building on our previous work on the coordination of a pair of mechanically decoupled vertical hoppers (A) De et al. (2018), we now introduce the slot hopper (B) as a new template for quadrupedal running. Its coordination stability analysis (Sec. 3), guides the synthesis of preflexive and feedback coordinated gaits in the progressively more complicated dynamics of the sagittal plane biped (C) and the physical Minitaur robot (D) Kenneally et al. (2016); Minitaur (2016) (Sec. 4) that robustly anchor this template (in the sense of Full and Koditschek (1999)) when properly compensated according to our controller designs. Fig. 18 and Table 3 summarize the relationships between these models, our controllers, and our analytical, numerical and empirical results.

the legs are coordinated into two pairs that seem to operate in synchrony Biewener (2003). Inspired by this phenomenon, engineers have formulated the concept of a virtual leg Sutherland and Carlson (1983); Raibert (1986), whereby groups of legs are "coordinated" such that the forces they exert on the body are equivalent to those that would have been exerted by a single virtual leg. The details of this coordination vary by implementation (ours is detailed in Sec. 4.2), but the goal is that the dynamical behavior of the system collapse down from their full state to a lower dimensional subspace well-represented by a monoped. Raibert et al. (1989) implemented all possible virtual bipedal quadruped gaits (Fig. 11) utilizing vertical energization of virtual legs, and often relying on preflexive out-of-phase coordination, which was systematically studied in simulation by Murphy and Raibert (1985). Berkemeier (1998) investigated this phenomenon further, and observed in simulation a second form of preflexive coordination (in-phase) in a verticallyrestricted model.

Prior analysis One appealing way to think abstractly about multi-legged locomotion is of the legs as abstract oscillators. This point-of-view permits ready formulation of gait coordination in terms of the oscillators' relative phases, as in Golubitsky et al. (1998). The work of Klavins et al. (2000) additionally shows how to construct these phase dynamics from some simple Hamiltonian mechanical systems, but that approach is more difficult to extend to non-Hamiltonian coupled dynamics required for implementation on the physical mechanism that we consider here. Klavins et al. (2002) numerically studied the coordination of two separate "bipedal" models using both feedforward ("central pattern generator, or CPG") and feedback schemes, finding that the feedback-generated oscillators were more robust to environmental uncertainty. Motivated by these results, we pursue feedback-only coordination here, and show analytically the stability of the resulting limit cycles with desired phase relations.

Other researchers have numerically studied coordination on simplified models of quadrupedal behavior: the pair of papers Poulakakis (2005) and Poulakakis (2006) study bounding on Scout II: an underactuated quadruped with fixed shank compliance and a single rotary actuator at each hip, which precludes the vertical energization scheme of Sec. 1.1. The authors explored the stability of a bounding limit cycle by numerically evaluating the return map eigenvalues for a $3 \mathrm{DOF}$ sagittal plane model (identical to Fig. 1C). Shahbazi and Lopes (2016) introduced a "DualSLIP" model of informationally-coupled, mechanicallydecoupled hoppers, and showed numerically that it was possible to coordinate them into pronking and bounding limit cycles by directly specifying the desired touchdown and liftoff times for each hip using a once-per-stride optimized controller. Our modeling paradigm is similar; the analysis in our companion paper De et al. (2018) provably attains similar coordination between two (now vertically restricted) hoppers with simple feedback laws. Gan et al. (2016) found numerical evidence of periodic orbits in a planar quadrupedal Hamiltonian model by optimizing body parameters, and relying on preflexive coordination.

Our analytical work is most closely related to Berkemeier (1998), who computes approximate return maps of inplace bounding and pronking using Raibert's (piecewiseHamiltonian) shank energization controller, and an $\varepsilon$ perturbation analysis. However, a full stability analysis for $\varepsilon \neq 0$ (including important nonlinear dissipation terms that contribute prominently to our stability proofs of vertical hopping as well as preflexive pronking) was not possible due to analytical intractability. In this paper, we bring new analytical tools to bear on this problem that are sufficiently powerful to provide a full stability analysis with a new nonlinear shank energization controller. 
To our knowledge this paper presents the first instances of stability proofs of any (both in-phase and out-of-phase) coordination schemes that bear directly on gaits on a physical quadruped-connections which we highlight in Table 3 .

Recent robotics Both of the contrasting feedforward/feedback approaches mentioned in Sec. 1.1 appear in empirical quadrupedal demonstrations in the literature. Righetti and Ijspeert (2008) optimize parameters for a CPG whose outputs are used to set the motor control signals. This technique has been empirically shown to propel some quadrupeds at speeds of 5-7 body lengths/second (see Fukuoka and Kimura (2009) and Sprowitz et al. (2013), though the latter results are from a $1 \mathrm{~kg}$ prototype without onboard power). However, purely open-loop control signals are particularly sensitive to perturbations from the environment Klavins et al. (2002), and also do not provide any analytical affordance since the body dynamics are invisible in the gait design space.

Some researchers eschew the CPG and directly optimize some parameterization of the motor signals: Shkolnik et al. (2011) use pre-planned position trajectories using an offline planning phase for a specific terrain which is assumed known a-priori. Pusey et al. (2013) use timing parameters which are optimized by hand to demonstrate feedforward bounding at a given speed. Culha and Saranli (2011) use an open-loop hip retraction scheme and passive shank to reduce the number of control parameters, add a spine joint for thrust, and numerically optimize gait parameters using a Nelder-Mead optimization step. However, in these purely open-loop implementations, the parameters are sensitive to desired speed, and the stability basin (in the presence of perturbations from external forces or rough terrain) is small. Hutter et al. (2014) introduce "operational space control", and the recent preprint from Neunert et al. (2016) brings model-predictive control (MPC) to bear on a physical quadruped. These papers are representative of a recent trend toward applying (generally highly complex) decision-space optimization methods to gait generation. MPC-like methods alleviate the instability problems of open-loop strategies over short time horizons by optimizing online over short horizons, but have been computationally prohibitive for all but the simplest examples, generally need very accurate models of the robot, and preclude any analytical insight. Barasuol et al. (2013) promote a combination of feedforward CPG-like trajectories augmented by feedback "reflexes" for walking and trotting. In the same vein, Park et al. (2017) demonstrate bounding at 6.5 body lengths/second on the $30 \mathrm{~kg}$ MIT Cheetah using feedforward vertical energization at the front and rear hips, and rely on preflexive coordination of the body pitch (which we analyze and

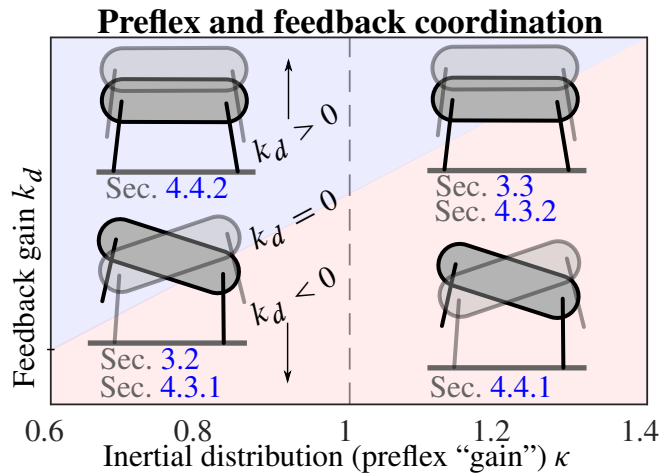

Figure 2. Phase locking in the slot hopper hopper template and its anchors depicted in Fig. 1. We show both analytically and empirically that the slot hopper-when controlled by almost independently energizing each leg as a vertical hopper-exhibits phase-locking to in-phase ("pronking") and anti-phase ("bounding") limit cycles. It does so in a manner affected by both its mechanical construction (non-dimensional inertia (13); horizontal axis) as well as a modest feedback controller applied to perturb the hopping frequencies (phase control gain (9); vertical axis), such that a desired behavior can be selected — with provable stability properties—either recruiting or countermanding preflexive behaviors. These parameters induce the analogous behaviors in the more complicated sagittal plane biped and physical minitaur (Fig. 1) as summarized in Fig. 18.

thereby predictively both countermand as well as reinforce in this paper).

From the perspective of control architecture and experimental practice, our work is arguably most similar to Raibert et al. (1989), whose empirical demonstration of virtual bipedal gaits mentioned above used systematic compositions of simple one DoF feedback controllers (and no feedforward signals). We have previously used similar compositions to demonstrate planar hopping in a tailed biped De and Koditschek (2015a), and also apply the same techniques to our quadruped here while requiring fewer actuators. We have included a careful comparison of specific aspects our control strategy to Raibert's in Table 5.

\subsection{Contributions and organization}

1.2.1 Contributions Fig. 1B introduces the "slot hopper" model (inspired by in-place biped models in prior literature, such as Burden et al. (2007); Berkemeier (1998)), wherein we add a notional mechanical coupling between the two independent vertical hoppers (Fig. 1A), taking the form of a connecting bar pivoting freely around a vertically-sliding pin. The central contribution of this paper as stated at the outset is the proposal of the slot hopper as a template for all "virtual bipedal" quadruped gaits, a stability analysis of the target limit cycles that encode them in that model, and the empirical demonstration of their anchoring in the 
physical Minitaur quadruped (Fig. 1D). The analysis and data presented in this paper to establish these claims is summarized in an "iconic" manner in Fig. 2 (and with supporting data later on in Fig. 18) as follows:

- In-place bipedal gait stability analysis. We provide the first correctness proofs for in-place dynamical virtual bipedal gaits by adapting the hybrid averaging analysis of De et al. (2018) to the three distinct (overlapping) physical regimes of the slot-hopper template determined by its "non-dimensional inertia" (13) as depicted along the abscissa of Fig. 2.

- Preflexive phase regulation and its systematic reassignment. In so doing, we show formally how these regimes elicit preflexive stabilization for both in-phase (pronking) and out-of-phase (bounding) quadrupedal hopping behavior in a manner determined by the sign of the coordination gain (9) as depicted along the ordinate of Fig. 2. Namely, we interpret the slot-hopper template's provably stable limit cycles (whose relative phase is depicted by the shadowed bodies of Fig. 2 and plotted by the black dots in Fig. 18) as being empirically anchored by closely matching periodic motions of the appropriately loaded physical Minitaur (green and magenta vertical lines in Fig. 18). We further demonstrate that the formally established preflexive behavior can be robustly either countermanded or reinforced by simple sign adjustments to the coordination control law gains introduced in (9)(10) when applied to the slot-hopper template (again depicted by the shadowed bodies of Fig. 2 but plotted as blue and red dots in Fig. 18), to achieve correspondingly enhanced or disrupted empirical pronking or bounding on the putatively anchoring physical Minitaur (plotted as colored open diamonds in Fig. 18).

- Empirical composition of anchored templates. Using only shank extension actuators we apply the identical three parameter ${ }^{1}$ family of slot-hopper controllers (7)-(10) to the laterally paired hips of Minitaur and present empirical evidence that this anchors the template by demonstrating that all in-place virtual bipedal quadruped gaits on the physical machine arise from the corresponding template parameter settings, as summarized in Table 3. We empirically compose these vertical hopping coordination controllers with fore-aft and yaw controllers to get useful and robust locomotion performance (Table 4).

The stability results propose for the first time a formal explanation for preflexive in-place coordination, extending the results of Berkemeier (1998) to include $\varepsilon \neq 0$ dynamics (responsible for the stabilization of height in our vertical hopper controller in Sec. 2.1.3 and of phase difference in the strongly coupled hoppers of Sec. 3.3); they show in addition that the preflexes are effective for a vastly different shank energization strategy than Raibert's, as well as that these results bear out on a physical platform.

1.2.2 Organization Table 3 summarizes how our analytical results (including both preflexive and feedback stabilization) and the insights gained therein are brought to bear on Minitaur to endow it with the virtual bipedal gaits. In contrast, Table 4 summarizes our empirical forays (moving beyond the analytical scope of this paper, but anticipating formal analysis now in progress) into parallel composition in the sense of De and Koditschek (2015a) of these robust in-place virtual bipedal limit cycles with other templates. In Sec. 2.1, we first call out results from the companion paper De et al. (2018) that are used as analytical tools for the proofs here. We introduce (Sec. 2.2) the slot hopper model (Fig. 1B), describe its relationship to both the abstract pair of vertical hoppers (Fig. 1A), as well as the more complex sagittal plane biped model (Fig. 1C). In Sec. 3, after observing that the feedback stabilization results of De et al. (2018) extend directly to the slot hopper's decoupled regime (Sec. 3.1), we prove (Sec. 3.2-3.3) that as that template's non-dimensional inertia (13) is varied through the range in Table 3, we obtain preflexive stability of bounding and pronking (covering the gamut of interesting virtual bipedal limit cycles). As summarized in Fig. 18, the physical robot Minitaur (2016) exhibits the same preflexive stability, switching from bounding (Fig. 4) to pronking (Fig. 12) when its non-dimensional inertia is modified with appropriately placed "outrigger" masses, without any modification in the control signals. Our analytical results are subject to a set of simplifying assumptions whose validity with respect to the physical robot is summarized in Table 2 and whose fidelity to its sagittal plane Lagrangian model, Fig. $1 \mathbf{C}$, is further established by extensive numerical study (Figs. 47). Using numerical simulation, in Sec. 4.1 we establish a strong correspondence between our models/assumptions and the physical machine on one hand (Sec. 4.1.1-4.1.3), as well as between our analytical and simulation results on the other (Sec. 4.1.4-4.1.5). We introduce our empirical results on Minitaur by first detailing our implementation in Sec. 4.2. Using insights from our analytical results as laid out in Table 3, we demonstrate preflexive bounding and pronking (Sec. 4.3), and also countermand the natural pattern by applying phase-modifying feedback (9)-(10) (Fig. 13, Fig. 14). In addition to the in-place gaits just discussed, the robustness of our control strategies allow empirical composition (Sec. 4.5) with additional controllers to stabilize fore-aft, roll, and yaw DOFs, and results in bounding at speeds up to approximately 5 body lengths/second 
(Fig. 15), punctuated by stably recovered leaps of 2 bodyheights (Fig. 16). Further intuitive combinatorial pairings of Minitaur's hips using appropriate combinations of (9)-(10) yields in-place pacing (Fig. 23) and trotting (Fig. 21), and pronking leaps of 3 body-heights (Fig. 14).

In Sec. 5, we conclude with observations and remarks that are not yet formal but offer more context for the present contributions and give a sense of what promising steps lie ahead in our current and near future work.

\section{Modeling and theory}

First, in Sec. 2.1, we review the key contributions of our companion paper De et al. (2018), introduce in Sec. 2.2 the slot hopper template plant, derive its equations of motion in Sec. 2.2.1-2.2.3, and show in Sec. 2.2.4 that setting $\kappa=1$ effectively results in physically decoupled vertical hoppers (Fig. 1A). Henceforth, we refer to this as the "decoupled" instance of the slot hopper. In Sec. 2.2, we describe the procedure by which the template vertically hopping controller is reused in the slot hopper.

\subsection{Background: hybrid averaging theory and application}

2.1.1 Hybrid averaging In the companion paper De et al. (2018), we introduced an analytical tool for $\varepsilon$-perturbation stability analysis of hybrid limit cycles. In the Theorem below (and the remainder of the paper), $\varepsilon>0$ is a sufficiently small scalar, which we relate to the various physical parameters of the system in each application (e.g., Sec. 3.2).

We repeat the statement of the constant flow-time (switching systems) averaging theorem here for the convenience of the reader; for the proof (as well as the extension to event-based switching), please see the companion paper.

Theorem 1. Switching Averaging Theorem. Given the "original" and "averaged" switching systems of the forms

$$
\begin{array}{lll}
\dot{x}=\varepsilon f(x, t, \varepsilon), & \dot{\theta}=1, & x\left(T_{+}\right)=R(x(T)), \\
\dot{y}=\varepsilon \bar{f}(y), & \dot{\theta}=1, & y\left(T_{+}\right)=R(y(T)),
\end{array}
$$

where $\theta$ is reset to 0 by the switching event, we assert the following hypotheses on (2):

(i) if the $C^{r}$ (for $r \geq 2$ ) reset $R$ (allowed to vary with $\varepsilon$ ) satisfies: (a) $\mathrm{D} R(x)=S_{0}+\varepsilon S_{1}(x, \varepsilon)$ (with constant $S_{0}$ ); (b) $S_{0}$ is invertible, and its unity eigenvalues have diagonal Jordan blocks ${ }^{2}$, and

(ii) if there is a point $p_{0}$ such that: (a) it is an equilibrium of $\bar{f} ;(b) R\left(p_{0}\right)=p_{0}$; and (c) the averaged return map is hyperbolic at $p_{0}$, then there exists $\varepsilon_{0}>0$ such that, for all $0<\varepsilon \leq \varepsilon_{0}$, (1) possesses a unique hyperbolic periodic orbit, of the same stability type as $p_{0}$.

Intuitively, condition (i) asserts that the resetlinearization $\mathrm{D} R$ only has an $\mathcal{O}(\varepsilon)$-dependence on the state, thus ensuring that its spectrum does not vary drastically between the true and our approximated fixed point. Condition (ii) asserts that the averaged fixed point is structurally stable, thus ensuring that the stability properties of the original system match our approximation of it. As we show in De et al. (2018), this theorem can be used to obtain analytical representations of (approximations of) hybrid limit cycles appearing in legged locomotion as well as their stability properties. The extensions of De et al. (2018) to symmetry-factored return maps and near-simultaneous transitions are also utilized to apply the single-mode result to the bounding and pronking limit cycles of interest.

For each of the analyses, we choose to evaluate stability of a return map using a Poincaré section at the physical touchdown event. Thm. 1 requires a single continuous mode (single stance in Sec. 3.2, double stance in Sec. 3.3), and a reset map following it (for which we integrate trivial aerial dynamics in each analysis).

2.1.2 Vertical hoppers in phase/energy coordinates Consider a pair $(i \in\{1,2\})$ of vertical hoppers with a unit mass at height $z_{i}$, with nominal leg length $\rho$, and with stance ("stance" is when $z_{i}<\rho$ ) dynamics $\ddot{z}_{i}=u_{i}$ and flight dynamics $\ddot{z}_{i}=-g$; i.e., we assume that in stance, there is a vertical extension actuator able to exert any desired force $u_{i}$ on the ground, and in flight, the mass follows ballistic flight.

We now present "phase/energy coordinates" for both its stance and flight phases. To that end, we define the phase vector, $p: \mathbb{R}^{2} \rightarrow \mathbb{R}^{2}$ corresponding to its vertical height in stance,

$$
p\left(z_{i}, \dot{z}_{i}\right):=\left[-\dot{z}_{i},\left(\rho-z_{i}\right) \omega\right]^{T},
$$

where $\omega$ is the natural frequency of the spring-mass system in stance (cf. (8)). The energy coordinate (intuitively changing slowly) in each mode, whose closest prior analogue in the literature is in Koditschek and Buehler (1991), is defined as

$$
a_{i}:= \begin{cases}\left\|p\left(z_{i}, \dot{z}_{i}\right)\right\| & z_{i}<\rho, \text { and } \\ \sqrt{2 g\left(z_{i}-\rho\right)+\dot{z}_{i}^{2}} & \text { else, }\end{cases}
$$

and the phase angle coordinate, inspired by Klavins et al. (2000) and intuitively with fast dynamics relative to the energy, is defined as

$$
\psi_{i}:= \begin{cases}\angle p\left(z_{i}, \dot{z}_{i}\right) & z_{i}<\rho, \text { and } \\ \frac{a_{i}-\dot{z}_{i}}{2 a_{i}} & \text { else. }\end{cases}
$$


Table 1. Table of important symbols used throughout the paper, listed by order of appearance, some originating in the companion paper De et al. (2018), others introduced (or redefined) in this paper.

\begin{tabular}{|c|c|c|c|c|c|}
\hline Symbol & Brief description & De et al. (2018) & Symbol & Brief description & De et al. (2018) \\
\hline$K: \mathbb{R}^{4} \rightarrow \mathbb{R}^{4}$ & Bipedal symmetry & (48) & $\bar{K}=\left[\begin{array}{lll}0 & 1 & 0 \\
1 & 0 & 0 \\
0 & 0\end{array}\right]$ & Slow-coord. symmetry & (49) \\
\hline$\omega \in \mathbb{R}_{+}$ & Stance spring frequency & (32) & $\omega_{\mathrm{f}}: a_{i} \mapsto g /\left(2 a_{i}\right)$ & Flight frequency & (35) \\
\hline Symbol & Brief description & This paper & Symbol & Brief description & This paper \\
\hline$\kappa \in \mathbb{R}_{+}$ & Non-dimensional inertia & (13) & $z_{i} \in \mathbb{R}$ & Physical hip height & $\S 2.1 .2$ \\
\hline$z \in \mathbb{R}$ & Physical CoM height & $\S 2.2$ & $\phi \in \mathbb{R}$ & Physical "body angle" & $\S 2.2$ \\
\hline$m_{b} \in \mathbb{R}_{+}$ & Mass of robot & $\S 2.2$ & $i_{b} \in \mathbb{R}_{+}$ & Rotational inertia of robot & $\$ 2.2$ \\
\hline$d \in \mathbb{R}_{+}$ & Half hip-to-hip distance & $\S 2.2$ & $a_{i} \in \mathbb{R}_{+}$ & Hip energy (liftoff vel.) & (4) \\
\hline$\psi_{i} \in \Psi \subset \mathbb{R}_{+}$ & Hip phase & (5) & $\beta \in \mathbb{R}_{+}$ & Stance damping & (8) \\
\hline$u_{i}: x \rightarrow \mathbb{R}$ & Hip control input & (7) & $v_{i}: X \rightarrow \mathbb{R}$ & Nonlinear part of control & (8) \\
\hline$w_{i}: X \rightarrow \mathbb{R}$ & Phase control & (8) & $k_{a} \in \mathbb{R}_{+}$ & Vertical gain & (8) \\
\hline$k_{p}, k_{d} \in \mathbb{R}_{+}$ & Coordination control gains & $(9),(10)$ & $\alpha_{i} \in \mathbb{R}$ & Morphological parameters & (18) \\
\hline$\Psi \subset \mathbb{R}$ & Phase space & $\S 2.1$ & $x \approx \mathbb{R}^{4}$ & Averaging domain & $\S 2.1$ \\
\hline$\varepsilon \in \mathbb{R}_{+}$ & Averaging parameter & $\S 2.1$ & $F: X \rightarrow T X$ & Stance vector field & $\S 2.1$ \\
\hline$f: x \rightarrow T x_{2}$ & Slow vector field & $\S 2.1$ & $\bar{f}: x_{2} \rightarrow T x_{2}$ & Averaged vector field & $\S 2.1$ \\
\hline $\bar{R}: x_{2} \rightarrow x_{2}$ & Slow coordinate reset & $\S 2.1$ & $\underline{\gamma}: x \rightarrow \mathbb{R}$ & Event function & $\S 2.1$ \\
\hline $\mathcal{G} \subset x$ & Guard set & $\S 2.1$ & $\underset{P}{\bar{P}}: x_{2} \rightarrow x_{2}$ & Averaged return map & $\S 2.1$ \\
\hline$\widetilde{\psi}_{i} \in \Psi \subset \mathbb{R}_{+}$ & Modified $\S 3.2$ hip phase & (27) & $\widetilde{\delta} \in \mathbb{R}$ & Modified $\S 3.2$ phase diff. & (28) \\
\hline$\left(\psi_{1}, a_{1}, a_{2}, \widetilde{\delta}\right)$ & "WC" averaging vector & $\S 3.2$ & $\zeta_{i} \in \mathbb{R}$ & Return map parameters & (33), (36) \\
\hline$\left(\psi_{1}, a_{1}\right)$ & "SC" averaging vector & $\$ 3.3 .3$ & $a_{\phi} \in \mathbb{R}_{+}$ & Body angle energy & (37) \\
\hline$a_{z} \in \mathbb{R}_{+}$ & CoM vertical energy & $(38)$ & $\omega_{\phi}, \beta_{\phi} \in \mathbb{R}_{+}$ & $\phi$-oscillation reparam. & (46) \\
\hline$Q_{\phi}: T S^{1} \rightarrow T S^{1}$ & $(\phi, \dot{\phi})$ stance flow & (43) & $R_{\phi}: T S^{1} \rightarrow T S^{1}$ & $(\phi, \dot{\phi})$ reset & (44) \\
\hline$t_{\mathrm{s}} \in \mathbb{R}_{+}$ & Stance duration & $\S 3.3$ & $t_{\mathrm{f}} \in \mathbb{R}_{+}$ & Flight duration & $\S 3.3$ \\
\hline
\end{tabular}

In order to study phase coordination between the two hoppers, we introduce a new "bounding phase difference" to compare $\psi_{1}$ to $\psi_{2}$ when hopper 1 is in stance and hopper 2 is in flight.

$$
\begin{aligned}
\delta & :=\tau_{1}-\tau_{2}, \text { where } \\
\tau_{i} & := \begin{cases}\left(\psi_{i}-\pi / 2\right) / \omega & z_{i}<\rho, \text { and } \\
\left(\psi_{i}-1 / 2\right) / \omega_{\mathrm{f}}\left(a_{i}\right) & \text { else, }\end{cases}
\end{aligned}
$$

where $\omega_{\mathrm{f}}$ is the "flight frequency" for the ballistic mass, as defined in Table 1.

2.1.3 Feedback controllers For our vertical extension actuator, we utilize the vertical hopping feedback controller from our own prior work De and Koditschek (2015a), and related to the tunable damping strategy of Secer and Saranli (2013),

$$
u_{i}(x):=\omega^{2}\left(\rho-z_{i}\right)+\varepsilon v_{i}(x),
$$

where the first summand is a Hooke's Law spring force (instantiated using actuators in our physical platform), and the second is a weak nonlinear forcing term comprising a lumped stabilizing (mass-normalized) damping term, given by $\beta$ in units of $\mathrm{N} /(\mathrm{m} / \mathrm{s}) / \mathrm{kg}$, as well as an active damping term modulated by gain $k_{a}$,

$$
v_{i}(x):=-\beta \dot{z}-k_{a} \cos \psi_{i}+w_{i}(x) .
$$

Intuitively, these terms modulate the hopping energy, with $\beta$ representing inescapable dissipation present on physical systems, and $k_{a}$ representing energy injection through feedback control. Finally, $w_{i}(x)$ is a phase coordination controller used to (informationally, through software) couple the two vertical hoppers $z_{1}$ and $z_{2}$. In De et al. (2018), we introduced two new intuitively-motivated coordination controllers: first, a "phase controller" which is a function of the velocity difference $\dot{z}_{1}-\dot{z}_{2}$.

$$
w_{i}(x):=(-1)^{i-1} k_{d}\left(\dot{z}_{1}-\dot{z}_{2}\right) \sin \psi_{i}
$$

As we show in De et al. (2018), the averaged effect of the control strategy (9) is akin to servoing on the abstract relative phase $\delta$. We also introduced an alternative "attitude controller"

$$
w_{i}(x):=(-1)^{i-1}\left(k_{p}\left(z_{1}-z_{2}\right)+k_{d}\left(\dot{z}_{1}-\dot{z}_{2}\right)\right),
$$

which has the more readily apparent effect of stabilizing $z_{1}-z_{2}$ in double stance when applied to both hoppers. Intuitively, it plays the role of a virtual attitude-stabilization impedance as in Park et al. (2017), and we show formally its effect in our analysis (Sec. 3.3).

2.1.4 Equilibria in oscillator coordinates We review from the analysis of mechanically-uncoupled vertical hoppers in De et al. (2018), the equilibrium of the averaged vector field

$$
\sigma^{*}=\pi, \quad x^{*}=\left[k_{a} / \beta, k_{a} / \beta, 0\right]^{T},
$$


and the reset map

$$
\bar{R}\left(\left[\begin{array}{c}
a_{1} \\
a_{2} \\
\delta
\end{array}\right]\right)=\bar{K}\left[\begin{array}{c}
a_{1} \\
a_{2} \\
\delta+\frac{a_{2}-a_{1}}{g}
\end{array}\right]=\left[\begin{array}{c}
a_{2} \\
a_{1} \\
-\delta+\frac{a_{1}-a_{2}}{g}
\end{array}\right] .
$$

\subsection{Linearized 2DOF slot hopper}

In prior literature, there are several instances of a $2 \mathrm{DOF}$ vertically-constrained biped (Fig. 1B), such as Burden et al. (2007); Berkemeier (1998). We include a complete Lagrangian derivation of its equations of motion ${ }^{3}$ below, and also derive a simplified analytically tractable version subject to an assumption that we present below (similar to the small-angle assumption made by Berkemeier (1998)).

Define the "non-dimensionalized inertia" parameter (originally due to Murphy and Raibert (1985)) which we shall henceforth refer to simply as the "inertia parameter",

$$
\kappa:=\frac{i_{b}}{m_{b} d^{2}},
$$

where $m_{b}$ is the body mass (without loss of generality, we set to $m_{b}=1 \mathrm{~kg}$, and we justify doing so in Sec. 2.2), $d$ is half the hip-to-hip distance and $i_{b}$ is the rotational inertia about the center of mass.

The configuration variables (including those for massless limbs) are $\mathbf{q}:=\left[r_{1}, r_{2}, z, \phi\right]^{T}$, where $r_{i} \in \mathbb{R}_{+}$are the lengths of the legs, $z \in \mathbb{R}_{+}$is the height of the center of mass, and $\phi$ is the "body angle." Despite the four entries, the number of dynamical DOFs accounting for constraints and degeneracies is only two, as shown below. The kinetic and potential energies are respectively

$$
T(\mathbf{q}, \dot{\mathbf{q}}):=\frac{1}{2} m_{b} \dot{z}^{2}+\frac{1}{2} i_{b} \dot{\phi}^{2}, \quad V(\mathbf{q}):=m_{b} g z .
$$

Actuation enters this model only through the stance legs, i.e. $\Upsilon_{i} \in \mathbb{R}$ are the forces acting on the $r_{i}$ prismatic joints. The model has two possible contacts - the two toes located at

$$
\mathbf{a}_{i}(\mathbf{q}):=z+(-1)^{i-1} d \sin \phi-r_{i}
$$

The following assumption allows us to derive simplified equations of motion for the slot hopper,

Assumption 1. To derive the constrained slot hopper dynamics (21), (22), we substitute the linearized version,

$$
\overline{\mathbf{a}}_{i}(\mathbf{q}):=z+(-1)^{i-1} d \phi-r_{1},
$$

of the exact constraint in (14).

As a plot of bounding data from the physical platform shows (Fig. 15), the oscillation in the body angle for Minitaur bounding is in the order of \pm 0.3 radians, where the small angle approximation error is less than $1.5 \%$.
Secondly, since our proofs only provide sufficient (not necessary) conditions for stability, we often find that the basin of stability is much larger than the scope of the analysis (whether it be in relation to Assumption 1, or the magnitude of the perturbation parameter). We compare the behavior of this simplified model to a higher fidelity hybrid system simulation as well as data from Minitaur in Fig. 4 to corroborate the validity of this assumption.

Lastly, define the virtual leg index set $\mathcal{J} \approx \mathbb{Z}_{2}$, where the members $i \in \mathcal{J}$ are " 1 " and " 2 ", but we use " $i-1$ " and " $i+1$ " to refer to the other element in the set. Also, define $z_{i} \in \mathbb{R}_{+}$, the heights of the hips, as

$$
z_{i}:=z+(-1)^{i-1} d \phi
$$

(anticipating the imposition of assumption 1). Clearly

$$
2 d \dot{\phi}=\dot{z}_{1}-\dot{z}_{2}
$$

which we use to change coordinates in Sec. 2.2.3. In the following, we will think of an abstract "hopper $i$ " being located at height $z_{i}$.

The continuous dynamics vary according to which constraints from (15) are active (physically corresponding to one or both toes contacting the ground). The different "modes" are thus: "double stance" (both $\overline{\mathbf{a}}_{i}$ active), "single stance" (only one active), and "aerial" (no constraints).

Controller parameter scaling We endeavor to apply the same scalar control feedback signals developed for vertical hopper control (recorded in (7)-(10)) to the slot hopper analyses (Sec. 3), simulations (Sec. 4.1), and Minitaur experiments (Sec. 4.2). This "template" controller feedback law, $u_{i}: X \rightarrow \mathbb{R}$, is related to the physical input signal $\Upsilon_{i} \in \mathbb{R}$ (Sec. 2.2) through

$$
\Upsilon_{i}(x):=\alpha_{0}+\alpha_{1} u_{i}(x),
$$

where $\alpha_{i} \in \mathbb{R}$ are constant parameters that we modify according the physical parameters of various plant models considered in this paper (Table 3). The formal effect of this affine transform (i.e., its role in facilitating the proofs of Sec. 3) is to cancel extraneous constant terms in the equations of motion (effectively mass and gravity-like parameters) and reduce to a form close to (25). This is a kind of morphological reduction as defined in Libby et al. (2015), however the details and physical implications of the reduction map are beyond the scope of this paper.

Lagrangian setup For the derivation of the equations of motion, we use the notation of Johnson et al. (2016). In each hybrid mode (subsections below) we define (a) the configuration variables $\mathbf{q}$, (b) the unconstrained (aerial) inertia tensor $\mathbf{M}(\mathbf{q})$, (c) the Lagrangian constraint $\mathbf{a}(\mathbf{q})$, whose rows are comprised of the exact "toe pinned" constraint (14), and (d) the input vector $\Upsilon$. Let $\mathbf{A}:=$ 
$\mathrm{D}_{\mathbf{q}} \mathbf{a}(\mathbf{q})$. Note that the gravity-like potential is the same in each case, and so the gravity-like forces are $\mathbf{N}(\mathbf{q})=$ $\mathrm{D}_{\mathbf{q}} V(\mathbf{q})=\mathrm{D}_{\mathbf{q}}\left(m_{b} g z\right)$. Lastly, the unconstrained inertia tensor happens to be constant in each case below, and so as in Johnson et al. (2016), we get the equations of motion from

$$
\left[\begin{array}{cc}
\mathbf{M} & \mathbf{A}^{T} \\
\mathbf{A} & 0
\end{array}\right]\left[\begin{array}{c}
\ddot{\mathbf{q}} \\
\lambda
\end{array}\right]=\left[\begin{array}{c}
\Upsilon-\mathbf{N} \\
\dot{\mathbf{A}} \dot{\mathbf{q}}
\end{array}\right]
$$

where $\lambda$ are the constraint forces at the toe. Lastly, in each case below, we use a text color to highlight terms in the equations of motion that are present without Assumption 1. When Assumption 1 is used, these terms get simplified as $\sin \phi, \cos ^{2} \phi$, and $\dot{\phi}^{2}$. Note that we prefer to impose the "small angle" approximation before taking derivatives (following Assumption 1) rather than after (which requires making the substitutions described in this paragraph and the following subsubsections). We include the dynamics absent Assumption 1, below, for completeness.

2.2.1 Single stance Without loss of generality, we consider the case where hopper 1 is in stance, and hopper 2 is in flight. The toe of hopper 1 is pinned to the ground, and so only constraint 1 is active in (15), or $\mathbf{a}(\mathbf{q})=\overline{\mathbf{a}}_{1}(\mathbf{q})$.

Without the small angle approximation, in single stance, we have $\mathbf{q}=[r, z, \phi], \mathbf{M}(\mathbf{q})=\operatorname{diag}\left[0, m_{b}, \kappa m_{b} d^{2}\right]$, $\mathbf{a}(\mathbf{q})=z+d \sin \phi^{-\phi}-r, \Upsilon=\Upsilon_{1}$. We can solve for $\ddot{q}$ by inverting the block matrix on the left of (19) (which is non-singular as long as there are no massless free DOFs or kinematic singularities Johnson et al. (2016)),

$$
\ddot{q}=\left[\begin{array}{c}
-g+\frac{\Upsilon_{1}}{m_{b}}\left(1+\frac{\left.\cos ^{2} \phi / \kappa\right)^{0}}{-d \sin \phi \dot{\phi}^{2} \eta^{0}}\right. \\
-g+\Upsilon_{1} / m_{b} \\
\frac{\Upsilon_{1} \cos \phi}{d m_{b} \kappa}
\end{array}\right]^{0}
$$

Even though the system has two dynamical DOF, we have three rows here, since the three (originally unconstrained) variables $r, z, \phi$ are now related through the toe-ground constraint. Additionally, to get the second row of (21), we use (16) with the first two rows of (20), and so the $\cos \phi$ in last row of (20) does not need to be approximated.

With the small angle approximation, using the change of coordinates from center-of-mass $(z, \phi)$ to "hip height" $z_{i}$ coordinates in (16), we get $m_{b} \ddot{z}=-m_{b} g+\Upsilon_{1}$, and $m_{b} \ddot{z}_{1}=-m_{b} g+(1+1 / \kappa) \Upsilon_{1}$. As per Sec. 2.2 and as listed in Table 3, we substitute (18), and get

$$
\ddot{z}_{1}=u_{1}, \quad \ddot{z}_{2}=-g-\frac{1-\kappa}{1+\kappa}\left(u_{1}+g\right),
$$

which can be compared to the exact version (20). We outline in Sec. 2.2 how the control input $u_{1}$ is set as a function of the robot state.

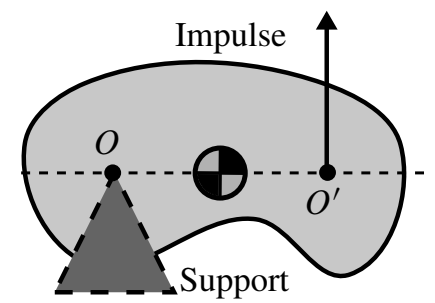

Figure 3. Center of percussion. From Symon (1971): "The point $O^{\prime}$ about which a blow must be struck in order that no impulse be felt at the point $O$ is called the center of percussion relative to $O$. If the body is unsupported, and is struck at $O^{\prime}$, its initial motion will be a rotation about $O$ ".

The physical implications of different values of $\kappa$ are noted in Table 3, and an intuitive interpretation of $\kappa$ is that it moves the center of percussion (see Fig. 3), where the net force on the hip over the stance duration plays the role of the "impulse." As (21) shows, a $\kappa=1$ configuration results in a complete lack of coupling interaction, equivalent to the "rotation about $O^{\prime}$ " condition of Fig. 3. In Table 3, we depict the location of the mass concentration (the location of the body mass if it were concentrated at a finite number of points, instead of distributed throughout the body, for equivalent total mass, inertia, and center-of-mass) as well as of the $\mathrm{CoP}$ as $\kappa$ is varied.

2.2.2 Double stance Without a small angle approximation, using $\quad \mathbf{q}=\left[r_{1}, r_{2}, z, \phi\right], \quad \mathbf{M}(\mathbf{q})=$ $\operatorname{diag}\left[0,0, m_{b}, \kappa m_{b} d^{2}\right], \quad \mathbf{a}(\mathbf{q})=\left[\begin{array}{c}z+d \sin \phi^{-\phi} r \\ z-d \sin \phi^{\phi}-r\end{array}\right], \quad$ and $\Upsilon=\left[\begin{array}{l}\Upsilon_{1} \\ \Upsilon_{2}\end{array}\right]$ in (19), we get

$$
\ddot{\mathbf{q}}=\left[\begin{array}{c}
-g+\frac{\Upsilon_{1}+\Upsilon_{2}}{m_{b}}+\frac{\left(\Upsilon_{1}-\Upsilon_{2}\right) \cos ^{2} \bar{\phi}^{0}}{m_{b} \kappa}-d \sin \phi \dot{\phi}^{2} \\
-g+\frac{\Upsilon_{1}+\Upsilon_{2}}{m_{b}}+\frac{\left(\Upsilon_{2}-\Upsilon_{1}\right) \cos ^{2}{ }^{0}}{m_{b} \kappa}+d \sin \phi \dot{\phi}^{2} \\
-g+\frac{\Upsilon_{1} \Upsilon_{2}}{m_{b}} \\
\frac{\left(\Upsilon_{1}-\Upsilon_{2}\right) \cos \phi}{d m_{b} \kappa}
\end{array}\right]^{0}
$$

Even though the system has two dynamical DOF, we have four rows here, since the four (originally unconstrained) variables $r_{1}, r_{2}, z, \phi$ are now related through two instances of the toe-ground constraint. Additionally, as above, the $\cos \phi$ in the last row does not need to be approximated when Assumption 1 is in place:

With the small angle approximation, after replacing $\Upsilon_{i}$ as in Table 3, using Assumption 1 we get:

$$
\begin{aligned}
m_{b} \ddot{z} & =-m_{b} g+\Upsilon_{1}+\Upsilon_{2}, \\
\ddot{\phi} & =\frac{\Upsilon_{1}-\Upsilon_{2}}{d m_{b} \kappa} .
\end{aligned}
$$


Table 2. Assumptions made to facilitate analytical results. We list how they are utilized, and also point to empirical or numerical justification.

\begin{tabular}{|c|c|c|c|}
\hline Assumption (title) & Application & Benefit & Justification \\
\hline $\begin{array}{l}\text { (De et al. 2018, 1) } \\
\text { (simultaneous transitions) }\end{array}$ & Sec. 3.3 & $\begin{array}{l}\text { apply switching averaging } \\
\text { (Thm. 1) }\end{array}$ & $\begin{array}{l}\text { Simulation comparison in Fig. } 7 ;<7 \% \\
\text { empirical single stance (Sec. } 4.1 .2 \text { ) }\end{array}$ \\
\hline $\begin{array}{l}\text { (De et al. 2018, 2) (constant } \\
\text { stance duration) }\end{array}$ & Sec. $3.2-3.3$ & simplify reset map & $\begin{array}{l}\text { Fig. } 15 \text { shows } 6.5 \% \text { deviation despite } \\
\text { perturbations (see Sec. A.5.1 discussion) }\end{array}$ \\
\hline $\begin{array}{l}1 \text { (linearized stance } \\
\text { constraint) }\end{array}$ & Sec. $3.2-3.3$ & $\begin{array}{l}\text { simplify single (21) and dou- } \\
\text { ble (22) stance dynamics }\end{array}$ & $\begin{array}{l}\text { Fig. } 5 \text { trajectory comparison } \\
\text { (with/without) }\end{array}$ \\
\hline 2 (low initial pitch energy) & Sec. 3.3 & $\begin{array}{l}\text { decouple the CoM and pitch- } \\
\text { ing dynamics (57) }\end{array}$ & $\begin{array}{l}\text { Fig. } 14 \text { shows relative magnitudes of } a_{\phi} \\
\text { and } a_{z} \text { (see Sec. } 4.4 .2 \text { discussion) }\end{array}$ \\
\hline 3 (small flight time) & Sec. 3.3 & $\begin{array}{l}\text { prove the time-varying } \phi \text { - } \\
\text { reset (44) is a contraction }\end{array}$ & $\begin{array}{l}\text { Only restricts analytical scope to low } \\
\text { energy pronking; Fig. } 9 \text { shows stability } \\
\text { for much higher } t_{\mathrm{f}} \text { (see } \mathrm{Sec} \text {. } 3.3 .6 \text { ). }\end{array}$ \\
\hline
\end{tabular}

Using Table 3 and substituting in (18), we get

$$
\ddot{z}=\frac{u_{1}+u_{2}}{2}, \quad \ddot{\phi}=\frac{u_{1}-u_{2}}{2 d \kappa} .
$$

2.2.3 Aerial In this case, Assumption 1 makes no difference. Using $\mathbf{q}=[z, \phi]$, no constraint $\mathbf{a}$, and no input $\Upsilon, \mathbf{M}(\mathbf{q})=\operatorname{diag}\left[m_{b}, \kappa m_{b} d^{2}\right], \ddot{\mathbf{q}}=\left[\begin{array}{c}-g \\ 0\end{array}\right]$ we get the equations

$$
\ddot{z}=-g, \quad \ddot{\phi}=0,
$$

and using (17) we can reinterpret these in the $z_{i}$ coordinates,

$$
\ddot{z}_{1}+\ddot{z}_{2}=-2 g, \quad \ddot{z}_{1}-\ddot{z}_{2}=0 \Longrightarrow \ddot{z}_{i}=-g,
$$

that is, the aerial phase is identical to the flight of two independent vertical hoppers (the reader may compare to De et al. (2018)).

2.2.4 Decoupled case First consider the stance dynamics of the decoupled $(\kappa=1)$ slot hopper. In double stance, using (16) in (22), and also setting $\kappa=1$, we get $\ddot{z}_{i}=$ $\ddot{z} \pm d \ddot{\phi}=u_{i}$, and in single stance, setting $\kappa=1$ in (21), we see that $\ddot{z}_{1}=u_{1}, \ddot{z}_{2}=0$.

In aerial phase, (23) is already decoupled. All together, we see that the decoupled slot hopper has the equations of motion (for each $i \in \mathcal{J}$ )

$$
\ddot{z}_{i}= \begin{cases}-g+u_{i} & \text { (stance) } \\ -g & \text { (flight). }\end{cases}
$$

\subsection{Phase differences and phase control}

The two coupled hips of the slot hopper are not identical oscillators, and we rely on intuition in each different nondegenerate regime in Sec. 3 to construct a modified phase difference coordinate (compared to (6)), such as (28) in Sec. 3.2.1. ${ }^{4}$

In addition to the feedback coordination controllers (9)-(10), the slot hopper exhibits preflexive phase control terms, i.e. their more complex equations of motion contain terms qualitatively resembling $w_{i}$ in (8) even without any feedback control. The specific form of $w_{i}$ in these cases is more difficult to express via a single equation, but we provide at the outset an intuitive description of the stability mechanism that is borne out by our analysis in this paper:

The weakly coupled hoppers are stabilized through a coupling interaction between the relative phase of the two hoppers and their energies (described in detail after (34)). Meanwhile, the strongly coupled hoppers are stabilized through the leg damping acting as a phase controller (cf. Sec. 3.3).

\section{Slot hopper analytical results}

First in Sec. 3.1, we discuss the $\kappa \approx 1$ "decoupled" regime of the slot hopper (as an extension of the $\kappa=1$ case idenified in Sec. 2.2.4). In Sec. 3.2-3.3, we set the input $w_{i} \equiv 0$ in (8)) of (a) out-of-phase hopping at $\kappa<1$ (which we term in the weak coupling regime in Table 3 ), and (b) inphase hopping at $\kappa \gg 1$ (which we term the strong coupling regime). ${ }^{7}$ In Sec. 3.3.7 we reintroduce phase control (9) and attitude control (10) through $w_{i}$, and show that it is possible to augment preflexive pronking with feedback. We present many more numerical and empirical instances of feedback augmentation/disruption of preflexive stability in the next section.

\subsection{The "decoupled" $\kappa \approx 1$ case}

In the companion paper, we analyzed the $\kappa=1$ case and saw that the return maps for bounding- and pronking-coordinated vertical hoppers are hyperbolic with eigenvalues $\mathcal{O}(\varepsilon)$ within the unit sphere. From the slot hopper dynamics in (21), (22), and (23), we see that the dynamics (specifically the coupling forces) depend smoothly on $\kappa$. Now suppose $\kappa=1+\mathcal{O}\left(\varepsilon^{2}\right)$. Then, coupling forces of $\mathcal{O}\left(\varepsilon^{2}\right)$ magnitude appear in the continuous dynamics, and are consequently ignored in the 
Table 3. Behavioral regimes and analytical results. As the inertia $\kappa(13)$ of a slot-hopper (Fig. 1B) is varied (mass concentration depicted as red disks) along the abscissa of the plot in Fig. 2 (and Fig. 18), we observe the following systematic changes in its morphological, analytical and behavioral features which are echoed by the sagittal plane biped (Fig. 1C) and Minitaur robot (Fig. 1D) in close correspondence: (a) the center of percussion (CoP, Fig. 3, depicted as blue disk) moves (cf. Sec. 2.2.1), (b) the preflexive control stabilizes different limit cycles (compare the analytical results depicted in Fig. 2 with the numerical summary of Fig. 18 whose superimposed markings locate the corroborating in-place empirical Minitaur experiments of Sec. 4.3.1-4.3.2), and (c) we are able to prove stability of five of these limit cycles (corresponding to the degenerate case, and either preflexive or feedback stabilization of both relative phase targets) as listed below.

\begin{tabular}{lllllll}
\hline Regime & $\begin{array}{l}\text { Relative } \\
\text { phase }\end{array}$ & $\begin{array}{l}\text { Coordination } \\
\text { control }\end{array}$ & $\alpha_{0}(18)$ & $\alpha_{1}(18)$ & Analysis & $\begin{array}{l}\text { Numerical/empirical } \\
\text { demonstration }\end{array}$ \\
\hline & anti-phase & $\begin{array}{l}\text { Preflexive } \\
(29),(34)\end{array}$ & $\frac{m_{b} g}{1+1 / \kappa}$ & $\frac{m_{b}}{1+1 / \kappa}$ & Peduces to single leg \\
De et al. (2018); see \\
Sec. 5.1.3
\end{tabular}

averaging step. Thus, the averaged return map is perturbed by $\mathcal{O}\left(\varepsilon^{2}\right)$, and the stability conclusions we have presented still hold.

\subsection{Weak coupling: preflex anti-phase stability}

In this subsection, we use $\varepsilon$ as a perturbation parameter perturbing both (a) the dissipation in the shank forcing controller (7), and (b) $\kappa$ (13), by setting $\kappa=\frac{1-\varepsilon}{1+\varepsilon}$. Specifically, we can reparameterize the dynamics (21) in terms of $\varepsilon=\frac{1-\kappa}{1+\kappa}$ to get

$$
\ddot{z}_{1}=u_{1}, \quad \ddot{z}_{2}=-(1+\varepsilon) g-\varepsilon u_{1} ;
$$

so that there is now a weak coupling interaction between the two hoppers for small $\varepsilon>0 .^{8}$

As in the prior bounding analysis, we capitalize on stepto-step symmetry and use the "half return map" analysis as depicted in the upper series of sketches in (De et al. 2018, Fig. 2), but supress the parenthetical subscripts, $F:=F_{(1)}, R:=R_{(1)}$ (as described in the last paragraph of Sec. 2.1) for ease of reading.
3.2.1 Continuous dynamics From (21), the stance dynamics of hopper 1 are free of any influence from hopper 2 , and so $\dot{a}_{1}, \dot{\psi}_{1}$ can be computed as for an isolated vertical hopper De et al. (2018). However, there is coupling from hopper 1 into the dynamics of hopper 2 , suggesting (for reasons to be motivated directly below in the discussion of (28)) the following coupled phase definition as a replacement for $\psi_{2}$ in (5):

$$
\widetilde{\psi}_{2}:=\psi_{2}+\frac{\varepsilon a_{1} \cos \psi_{1}}{2 a_{2}} .
$$

Owing to the new mechanical coupling, here we must define the following modified $\delta$ (specifically in the patch where hopper 1 is in stance and hopper 2 is in flight):

$$
\widetilde{\delta}:=\frac{\psi_{1}-\pi / 2}{\omega}-\frac{\widetilde{\psi}_{2}-1 / 2}{(1+\varepsilon) \omega_{\mathrm{f}}\left(a_{2}\right)}
$$

Again, this definition is motivated by the aim to find a "slow" coordinate, $\frac{d}{d t} \widetilde{\delta}=\mathcal{O}(\varepsilon)$, which is in equilibrium on the limit cycle. ${ }^{9}$ With these new definitions, using the flight 
energy coordinate from (4) in (21),

$$
\begin{aligned}
a_{2} \dot{a}_{2} & =\dot{z}_{2}\left(g+\ddot{z}_{2}\right)=-\varepsilon \dot{z}_{2}\left(u_{1}+g\right) \\
& \stackrel{(5)}{=}-\varepsilon a_{2}\left(1-2 \psi_{2}\right)\left(u_{1}+g\right) \\
& \stackrel{(7)}{=}-\varepsilon a_{2}\left(1-2 \psi_{2}\right)\left(\omega a_{1} \sin \psi_{1}+g\right)+\mathcal{O}\left(\varepsilon^{2}\right) \\
\Rightarrow \dot{a}_{2} & \stackrel{(27)}{=}-\varepsilon\left(1-2 \widetilde{\psi}_{2}\right)\left(\omega a_{1} \sin \psi_{1}+g\right)+\mathcal{O}\left(\varepsilon^{2}\right) .
\end{aligned}
$$

Using (28) to replace $\widetilde{\psi}_{2}$ with $\widetilde{\delta}$, we get

$$
\dot{a}_{2}=-\frac{g \varepsilon\left(2 \omega \widetilde{\delta}-2 \psi_{1}+\pi\right)\left(\omega a_{1} \sin \psi_{1}+g\right)}{2 \omega a_{2}}
$$

To calculate, $\frac{d}{d t} \widetilde{\delta}$, first we calculate $\frac{d}{d t} \widetilde{\psi}_{2}$. From (5), $\dot{\psi}_{2}=$ $-\frac{\ddot{z}_{2}}{2 a_{2}}+\frac{\dot{z}_{2} \dot{a}_{2}}{2 a_{2}^{2}}$. Using (26) and (29),

$$
\begin{array}{r}
\dot{\psi}_{2}=\frac{g}{2 a_{2}}+\varepsilon\left(\frac{g\left(g+u_{1}\right)\left(2 \psi_{2}-1\right)\left(2 \omega \widetilde{\delta}-2 \psi_{1}+\pi\right)}{4 \omega a_{2}^{2}}\right. \\
\left.+\frac{g+u_{1}}{2 a_{2}}\right)+\mathcal{O}\left(\varepsilon^{2}\right),
\end{array}
$$

and then using (7) and the newly defined phase coordinate (27),

$$
\begin{aligned}
& \frac{d}{d t} \widetilde{\psi}_{2}=\dot{\psi}_{2}-\frac{\varepsilon a_{1} \omega \sin \psi_{1}}{2 a_{2}}+\mathcal{O}\left(\varepsilon^{2}\right)=\frac{g}{2 a_{2}}+ \\
& \frac{\varepsilon g\left(\frac{\left(2 \psi_{2}-1\right)\left(2 \omega \widetilde{\delta}-2 \psi_{1}+\pi\right)\left(\omega a_{1} s_{1}+g\right)}{\omega}+2 a_{2}\right)}{4 a_{2}^{2}}+\mathcal{O}\left(\varepsilon^{2}\right) .
\end{aligned}
$$

Now we move on to using this to calculate $\frac{d}{d t} \widetilde{\delta}$. Using (28) and also substituting in $\dot{\psi}_{1}$, we get

$$
\frac{d}{d t} \widetilde{\delta}=\frac{\varepsilon \sin \psi_{1} v_{1}}{\omega a_{1}}+\mathcal{O}\left(\varepsilon^{2}\right) .
$$

Now we can complete the continuous dynamics for $\sigma:=$ $\psi_{1}, x:=\left(a_{1}, a_{2}, \widetilde{\delta}\right)$,

$$
\begin{aligned}
& \dot{\sigma}=\omega+\frac{\varepsilon}{a_{1}} \sin \psi_{1} v_{1} \\
& \dot{x}=\varepsilon\left[\begin{array}{c}
-\cos \psi_{1} v_{1} \\
\frac{-g\left(2 \omega \widetilde{\delta}-2 \psi_{1}+\pi\right)\left(\omega a_{1} \sin \psi_{1}+g\right)}{2 \omega a_{2}} \\
\frac{\sin \psi_{1} v_{1}}{\omega a_{1}}
\end{array}\right]=: \varepsilon f(x, \sigma, \varepsilon),
\end{aligned}
$$

where $v_{1}$ is defined in (8) but now time with a silenced active phase coordination term $w_{1} \equiv 0$. The form of (30) yields $\frac{d x}{d \sigma}=\mathcal{O}(\varepsilon)$ as required by Thm. 1. Comparing to the dynamics of the independent hoppers in De et al. (2018), we notice that $\dot{\sigma}, \dot{a}_{1}$ are identical (a consequence of the lack of coupling from hopper 2 to hopper 1 in (21)), and second, in sharp contrast, that the $\dot{a}_{2}$ dynamics (29) are intricately coupled with the phase difference $\widetilde{\delta}$.
Anticipating an anti-phase limit cycle, we consider the same $x^{*}$ as for the independent hoppers (11), and averaging (30) with respect to the fast variable $\sigma$ after substituting in (8) gives us

$$
\bar{f}(x)=\left[\begin{array}{c}
\frac{k_{a}-a_{1} \beta}{2 \omega} \\
\frac{-g \widetilde{\delta}\left(g \pi+2 a_{1} \omega\right)}{a_{2} \pi \omega} \\
0
\end{array}\right],
$$

which evaluates to 0 at $x^{*}$, satisfying Thm. 1(ii). Note that $\delta$ has an averaged effect on the energy level of the flight hopper (second row above). This departure from the mechanically decoupled hoppers De et al. (2018) contributes to the preflexive stabilization of the bounding limit cycle. Intuitively, if the flight hopper is leading in phase $\widetilde{\delta}<0), a_{2}$ is increased, and vice versa. This effect over stance interacts with the reset (see text after (34)). Moreover, we can calculate the Jacobian at the fixed point,

$$
\mathrm{D} \bar{f}\left(x^{*}\right)=\left[\begin{array}{ccc}
-\frac{\beta}{2 \omega} & 0 & 0 \\
0 & 0 & -g \zeta_{4} \\
0 & 0 & 0
\end{array}\right],
$$

where

$$
\zeta_{4}:=\frac{g \beta}{k_{a} \omega}+\frac{2}{\pi}
$$

is a scalar only depending on constant parameters.

3.2.2 Reset Since the aerial dynamics are the same as for isolated hoppers, the reset for the $a_{i}$ components are the same as in (12). We now calculate the reset for the last coordinate $\widetilde{\delta}$.

We use the explicit superscripts.$^{s}$ or ${ }^{f}$ to denote which coordinate patch (case of (5)) is being used. We use " $c_{1}$ " as shorthand for $\cos \psi_{1}^{\mathrm{s}}$ in this calculation. At liftoff,

$$
\begin{aligned}
\delta\left(t_{\mathrm{LO}}\right) & =\frac{\psi_{1}^{\mathrm{s}}\left(t_{\mathrm{LO}}\right)-\pi / 2}{\omega}-\frac{\psi_{2}^{\mathrm{f}}\left(t_{\mathrm{LO}}\right)-1 / 2}{\omega_{\mathrm{f}}\left(a_{2}\right)} \\
& \stackrel{(27)}{=} \frac{\psi_{1}^{\mathrm{s}}\left(t_{\mathrm{LO}}\right)-\pi / 2}{\omega}-\frac{\widetilde{\psi}_{2}\left(t_{\mathrm{LO}}\right)-1 / 2}{\omega_{\mathrm{f}}\left(a_{2}\right)}+\frac{\varepsilon c_{1} a_{1}}{g} \\
& \stackrel{(28)}{=} \widetilde{\delta}+\varepsilon\left(\widetilde{\delta}+\frac{c_{1} a_{1}}{g}-\frac{\psi_{1}^{\mathrm{s}}\left(t_{\mathrm{LO}}\right)-\pi / 2}{\omega}\right) .
\end{aligned}
$$

Similarly, but bearing in mind that at the touchdown patch hopper 1 is in flight and hopper 2 is in stance, and accordingly modifying (28), we get

$$
\begin{aligned}
\delta\left(t_{\mathrm{TD}}\right) & =\frac{\psi_{1}^{\mathrm{f}}\left(t_{\mathrm{TD}}\right)-1 / 2}{\omega_{\mathrm{f}}\left(a_{1}\right)}-\frac{\psi_{2}^{\mathrm{s}}\left(t_{\mathrm{TD}}\right)-\pi / 2}{\omega} \\
& \stackrel{(27)}{=} \frac{\psi_{1}^{\mathrm{f}}\left(t_{\mathrm{TD}}\right)-1 / 2}{\omega_{\mathrm{f}}\left(a_{1}\right)}-\frac{\psi_{2}^{\mathrm{s}}\left(t_{\mathrm{TD}}\right)-\pi / 2}{\omega}-\frac{\varepsilon c_{2} a_{2}}{g} \\
& \stackrel{(28)}{=} \widetilde{\delta}+\varepsilon\left(\widetilde{\delta}-\frac{c_{2} a_{2}}{g}+\frac{\psi_{2}^{\mathrm{s}}\left(t_{\mathrm{TD}}\right)-\pi / 2}{\omega}\right) .
\end{aligned}
$$

Using the boundary conditions $\psi_{1}^{\mathrm{s}}\left(t_{\mathrm{LO}}\right)=\pi$ (constant flow-time assumption in Table 2 ), and $\psi_{2}^{\mathrm{s}}\left(t_{\mathrm{TD}}\right)=0$ (initial 
condition for the flow of Thm. 1) in the equations above,

$$
\begin{aligned}
& \delta\left(t_{\mathrm{LO}}\right)=\widetilde{\delta}+\varepsilon\left(\widetilde{\delta}-\frac{a_{1}}{g}-\frac{\pi}{2 \omega}\right), \\
& \delta\left(t_{\mathrm{TD}}\right)=\widetilde{\delta}+\varepsilon\left(\widetilde{\delta}-\frac{a_{2}}{g}-\frac{\pi}{2 \omega}\right) .
\end{aligned}
$$

Substituting this into (12), and dividing through by $(1+\varepsilon)$,

$$
\widetilde{\delta}\left(t_{\mathrm{TD}}\right)=\widetilde{\delta}\left(t_{\mathrm{LO}}\right)+\frac{a_{2}-a_{1}}{g} .
$$

Putting everything together,

$$
\bar{R}\left(a_{1}, a_{2}, \widetilde{\delta}\right)=\bar{K}\left[a_{1}, \quad a_{2}, \quad \widetilde{\delta}+\frac{a_{2}-a_{1}}{g}\right]^{T} .
$$

We see that $x^{*}$ in (11) when substituted into the equation above yields $\bar{R}\left(x^{*}\right)=x^{*}$, and $\mathrm{D} \bar{R}$ has a constant $\mathcal{O}(1)$ part, together satisfying Thm. 1(i).

The influence of the energy levels $a_{i}$ on the phase difference $\widetilde{\delta}$ (apparent in the last entry above) results in a coupled interaction of $a_{2}$ and $\widetilde{\delta}$ through the continuous dynamics (31) and the reset (34). Intuitively, following along the example in the discussion after (31), if the flight hopper is leading in phase $(\delta<0)$, then $a_{2}$ is increased during stance (as discussed before). From (34), the flight hopper now has a shorter flight period, and so $\delta$ is increased.

3.2.3 Stability test Using (32) and (34), we can compute the averaged return map and evaluate at $x^{*}(11)$,

$$
\mathrm{D} \bar{P}\left(x_{2}^{*}\right)=\left[\begin{array}{cc|c}
0 & 1 & -\varepsilon g \zeta_{4} \\
\zeta_{5} & 0 & 0 \\
\hline \zeta_{5} / g & -1 / g & -\left(1-\varepsilon \zeta_{4}\right)
\end{array}\right],
$$

where $\zeta_{4}$ is defined in (33), and

$$
\zeta_{5}:=1-\frac{\varepsilon \beta}{2 \omega}
$$

As shown in A.1, the return map is hyperbolic, and so we can apply Thm. 1 to conclude that the weakly coupled hoppers have a stable anti-phase limit cycle $\varepsilon$-close to (11); we tabulate this result in Table 3 .

3.2.4 Disruption with feedback The preflexive stability we just proved is demonstrated in empirical results in Sec. 4.3, 4.5. We also demonstrate (though we do not analyze) the disruption of this preflexive stability using the phase controller (9) in Sec. 4.4.1. As argued in Remark 3.1, we expect the hyperbolic phase-controlled limit cycles to persist in a sufficiently small neighborhood of $\kappa$ around 1. In interests of space we omit a full analysis of the weakly coupled slot hopper as detailed in this section with $w_{i}$ set according to (9) (which would proceed almost identically to the proof presented here, with the incorporation of the feedback as shown in De et al. (2018)).

\subsection{Strong coupling: preflex in-phase stability}

In this subsection we consider the "strongly coupled" massparameter regime $\kappa \gg 1$ (last row of Table 3), where the body's mass concentration beyond the hips moves the center of percussion far from the mass center, thus diminishing the moment associated with differences in the ground reaction forces of one leg vs. the other. Unlike in Sec. 3.2, where we used an explicit analytical relation between $\kappa$ and $\varepsilon$ made in (21), $\kappa$ and $\varepsilon$ are unrelated in this section. The regime of operation here is one of "large $\kappa$," but still "small $\varepsilon . "$ As in De et al. (2018), we instead now interpret $\varepsilon$ as the magnitude of the (naturally ocurring) dissipative forces along with the underlying hopping energization terms (second summand in (8) originally introduced in De and Koditschek (2015a)) applied to the vertical hopper according to (7).

Because of the strong coupling, we find it helpful to work in the physical $(z, \phi)$ coordinates (center of mass location and body angle), subject to Assumption 1 as in Sec. 2.2.2. First, we introduce an assumption on the initial "attitude" energy:

Assumption 2. The initial conditions lie within the "low $a_{\phi} "$ set $\mathcal{L}:=\left\{x: a_{\phi} /\left(\kappa a_{z}\right)=\mathcal{O}\left(\varepsilon^{1 / 2}\right)\right\}$.

Our justification for the assumption is listed in the nextto-last row of Table 2; specifically, we show empirical evidence of its applicability in Fig. 14. It allows us to decouple the vertical and attitude dynamics (Sec. 3.3.2). Next, we show that the vertical dynamics are stable (Sec. 3.3.3), which is unsurprising since the decoupled dynamics resemble that of an isolated vertical hopper.

Then, in Sec. 3.3.4-3.3.6, we tackle stability of the "attitude" coordinate, $\phi$. First, we rigorously prove in Sec. 3.3.5 that the continuous-time orbits of $r_{\phi}$ followed by its resets render $\mathcal{L}$ (of Assumption 2) positive invariant, subject to the following additional assumption:

Assumption 3. The flight time is bounded above: $t_{\mathrm{f}}<$ $\varepsilon \pi \beta \sqrt{d / \kappa} / \omega^{2}$.

This assumption (and the contraction proof it enables) is necessary because the stability of the $\phi$-dynamics depend on the flight time of the slot hopper, thus rendering the $\phi$-return map time-varying. However, numerical and empirical exercise of all these models shows that the timevariation in the return map is indeed insignificant; we offer evidence that Assumption 3 is unnecessary both in simulation (Sec. 4.1.4), as well as in data taken from the physical Minitaur experiments summarized in Fig. 12. In Sec. 3.3.6, we discuss an assumed time-invariant version of the $\phi$-stability analysis, exposing the relation of the shape parameter $\kappa$ to the behavior of $\phi$, not subject to Assumption 3. 
3.3.1 Coordinate changes Similar to the map $p$ in (3), we define the " $\phi$-phase"

$$
p_{\phi}:=d\left[\begin{array}{ll}
-\dot{\phi}, & -\phi \omega
\end{array}\right]^{T}, \quad a_{\phi}:=\left\|p_{\phi}\right\|,
$$

as in (4). Unlike the complete polar transformation for the shank coordinate in (4) and (5), in this instance we only need the body angle energy $a_{\phi}$ for analysis. This is because in this subsection, the desired limiting behavior is a point attractor for $\phi$ (not an oscillatory behavior), and consequently a " $\phi$-phase" $\psi_{\phi}$ is neither well-defined nor required for analysis. In contrast, because vertical oscillations of the mass center are of key interest, we will need to define the vertical hopping energy and phase,

$$
a_{z}:=\|p(z, \dot{z})\|, \quad \psi_{z}:=\angle p(z, \dot{z})
$$

as in (4) and (5) respectively. Note that the vertical energy coordinate $a_{z}$ has units of $\mathrm{m} / \mathrm{s}$ as before in (4), and the new attitude energy coordinate $a_{\phi}$ also has the same units (since in (37), $d$ is a length, and $\phi$ is an angle).

3.3.2 Decoupling In A.2, we show how Assumption 2, empirically justified in the last row of traces in Fig. 14, allows us to decouple the $z$ and $\phi$ dynamics in stance to $\mathcal{O}(\varepsilon)$

$$
\begin{aligned}
\ddot{z} & =\omega^{2}(\rho-z)-\varepsilon \beta \dot{z}-\varepsilon k_{a} \cos \psi_{z}, \\
\kappa \ddot{\phi} & =-d\left(\omega^{2}+k_{p}\right) \phi-d\left(\varepsilon \beta+k_{d}\right) \dot{\phi},
\end{aligned}
$$

where $\psi_{z}=\angle p(z, \dot{z})$.

Additionally, note that the aerial dynamics in (23) are decoupled already, enabling completely isolated analyses of the vertical " $z$ " DOF (in Sec. 3.3.3) and the attitude " $\phi$ " DOF (in Sec. 3.3.4-3.3.7). ${ }^{10}$

3.3.3 CoM vertical dynamics stability The $z$ continuous dynamics (first row of (60)) are exactly the same as of a single "virtual" leg located at the center of mass Raibert (1986); Saranli and Koditschek (2003). The analysis of this subsystem proceeds identically to the analysis of a single vertical hopper De et al. (2018) (which utilizes Thm. 1), and we obtain the same stability result.

3.3.4 Attitude continuous dynamics: preflexive For Sec. 3.3.4-3.3.6, we examine preflexive stability by setting $k_{p}=k_{d}=0$ in (60). First we reparameterize the $\ddot{\phi}$ equation in (60) with

$$
\omega_{\phi}:=\omega \sqrt{d / \kappa}, \quad \beta_{\phi}:=\frac{\varepsilon \beta}{2 \omega} \sqrt{d / \kappa},
$$

to yield $\ddot{\phi}=-\omega_{\phi}^{2}\left(1+\beta_{\phi}^{2}\right) \phi-2 \omega_{\phi} \beta_{\phi} \dot{\phi}$. Using the linear coordinate change

$$
y_{\phi}:=\left[\begin{array}{cc}
\omega_{\phi} \sqrt{1+\beta_{\phi}^{2}} & \beta_{\phi} / \sqrt{1+\beta_{\phi}^{2}} \\
0 & 1 / \sqrt{1+\beta_{\phi}^{2}}
\end{array}\right]\left[\begin{array}{l}
\phi \\
\dot{\phi}
\end{array}\right]
$$

from (Koditschek and Buehler 1991, A.1), we get

$$
\dot{y}_{\phi}=\left(-\omega_{\phi} \beta_{\phi} I-\omega_{\phi} J\right) y_{\phi},
$$

where $J:=\left[\begin{array}{cc}0 & -1 \\ 1 & 0\end{array}\right]$ is the skew form of a rotation in the plane. Directly solving this linear system, we get the time$t_{\mathrm{s}}$ flow of the vector field (42),

$$
Q_{\phi}\left(y_{\phi} ; t\right)=e^{-\beta_{\phi} \omega_{\phi} t_{\mathrm{s}}} \operatorname{Rot}\left(-\omega_{\phi} t_{\mathrm{s}}\right) y_{\phi},
$$

where Rot : $S^{1} \rightarrow \mathbb{R}^{2 \times 2}: \theta \mapsto \exp (\theta J)$ is the rotation matrix on the plane.

The reset for the $\phi$ coordinates (from the decoupled $\ddot{\phi}=$ 0 aerial $\phi$-dynamics) is a "time of flight" $t_{\uparrow}$-parameterized map,

$$
R_{\phi}\left(y_{\phi}\right)=\left(I+\omega_{\phi} t_{\mathrm{f}} N\right) y_{\phi}, \text { where } N:=\left[\begin{array}{ll}
0 & 1 \\
0 & 0
\end{array}\right] .
$$

3.3.5 Attitude stability: preflexive Since the $\phi$-dynamics are decoupled from the $z$-dynamics to $\mathcal{O}(\varepsilon)$ in both the flow (60) and reset (44), we can analyze the $\phi$-stability separately. Direct computation yields the return map

$$
\begin{aligned}
P_{\phi}\left(y_{\phi}\right) & =R_{\phi} \circ Q_{\phi}\left(y_{\phi} ; t_{\mathrm{s}}\right) \\
& =e^{-\beta_{\phi} \omega_{\phi} t_{\mathrm{s}}}\left(I+\omega_{\phi} t_{\mathrm{f}} N\right) \operatorname{Rot}\left(-\omega_{\phi} t_{\mathrm{s}}\right) y_{\phi} .
\end{aligned}
$$

We show in A.3 that $P_{\phi}$ is a contraction. The continuous $\phi$ dynamics already (60) strictly contract $a_{\phi}$, and so $\mathcal{L}$ is positive invariant.

In summary, the decoupling described in Sec. 3.3.2, the proof of vertical stability of the CoM in Sec. 3.3.3, and the proof of $\phi$-stability in this subsection together guarantee stability of the in-phase limit cycle; we list this result in Table 3. We continue below to make further observations about the behavior when Assumption 3 is not made, or in the presence of feedback.

3.3.6 Attitude stability: time-invariant, preflexive Making instead the approximation that both the stance time $t_{\mathrm{s}}$ and flight time $t_{\mathrm{f}}$ are constant (justified by observing their small variance in empirical trials such as the one in Fig. 12), we now have a time-invariant $\phi$-return map (45), and we can look at the eigenvalues of $P_{\phi}$ to discern stability.

Define $\widetilde{P}_{\phi}:=\left(I+\theta_{\mathrm{f}} N\right) \operatorname{Rot}\left(-\theta_{\mathrm{s}}\right)$ such that $P_{\phi}\left(y_{\phi}\right)=$ $e^{-\beta_{\phi} \theta_{\mathrm{s}}} \widetilde{P}_{\phi} y_{\phi}$. Note that

$$
\operatorname{det} \widetilde{P}_{\phi}=1, \quad \operatorname{tr} \widetilde{P}_{\phi}=2 \cos \theta_{\mathrm{s}}-\theta_{\mathrm{f}} \sin \theta_{\mathrm{s}} .
$$

Next, we prove and use the following Lemma:

Lemma 1. If $\operatorname{det} \widetilde{P}_{\phi}=1$ and $\left|\operatorname{tr} \widetilde{P}_{\phi}\right| \leq 2$, then $\widetilde{\lambda}_{i}$, the eigenvalues of $P_{\phi}$, are complex conjugate with unit magnitude.

Proof. Since the determinant is identically $1, \widetilde{\lambda}_{i}=\operatorname{tr} / 2 \pm$ $\sqrt{(\operatorname{tr} / 2)^{2}-1}=: \xi_{0} \pm j \xi_{1}$, and $\left|\widetilde{\lambda}_{i}\right|^{2}=\xi_{0}^{2}+\xi_{1}^{2}=1$. 
Note from (40) that $\omega_{\phi} \propto 1 / \sqrt{\kappa}$, and so when $\kappa$ is made arbitrarily large, $\left|\operatorname{tr} \widetilde{P}_{\phi}\right| \rightarrow 2$. The eigenvalues of $P_{\phi}$ are $\lambda_{i}=e^{-\beta_{\phi}} \theta_{\mathrm{s}} \widetilde{\lambda}_{i}$, and so (using the Lemma above) $\lambda_{i}$ lie within the unit circle. We conclude that the return map $P_{\phi}$ is stable, without requiring the restrictive Assumption 3.

We show numerically (in Sec. 4.1.4) as well as empirically, by mechanically reconfiguring Minitaur to have two different $\kappa$ values (in Sec. 4.3), that this condition on $\kappa$ is important, and that instability of the in-phase limit cycle results when $\kappa$ is not large enough. In other words, the "weakly coupled" body of Sec. 3.2 (as well as our physical platform Minitaur arranged with its "native" intended body mass distribution) does not possess a stable in-phase limit cycle, and requires feedback control (which we discuss next) to render it attracting, thereby eliciting a pronk.

3.3.7 Attitude stability: feedback Now, we augment the preflexive stability to reinforce the in-phase (pronking) limit cycle by reintroducing feedback through positive $k_{p}$ and $k_{d}$ in (10). From (60), we have enough control affordance to stabilize the $\phi$ dynamics to a point within a single stance phase as follows.

First, we reparameterize the constants $k_{p}$ and $k_{d}$ in terms of a new parameter, $k_{\phi}>0$, such that

$$
\frac{d\left(\omega^{2}+k_{p}\right)}{\kappa}=k_{\phi}, \quad \frac{d\left(\varepsilon \beta+k_{d}\right)}{\kappa}=\frac{k_{\phi}^{2}}{2} .
$$

Using this in the second row of (60), we get

$$
\ddot{\phi}=-k_{\phi} \dot{\phi}-k_{\phi}^{2} \phi / 2 \text {. }
$$

Now define the "graph error" Koditschek (1987) $e_{\phi}:=$ $\dot{\phi}+k_{\phi} \phi$. Using (46), we get

$$
\dot{e}_{\phi}=-2 k_{\phi} e_{\phi} \Longrightarrow e_{\phi}(t):=e^{-2 k_{\phi} t} e_{\phi}(0),
$$

and so $k_{\phi}$ can be made arbitrarily large to get $e_{\phi}(t)$ arbitrarily small in stance. Consequently, in arbitrarily small time, the flow collapses to the $e_{\phi}^{-1}(0)$ submanifold, where by definition of $e_{\phi}$,

$$
\dot{\phi}=-k_{\phi} \phi \Longrightarrow \phi(t)=e^{-k_{\phi} t} \phi(0),
$$

and with large $k_{\phi}$, both $\phi(t), \dot{\phi}(t)$ are also driven to zero arbitrarily fast. Denote this deadbeat stance map as

$$
Q_{\phi, \mathrm{FB}}:(\phi, \dot{\phi}) \mapsto \text { “arbitrarily close to } 0 ” .
$$

Choosing a Poincaré section at liftoff, we get the return map

$$
P_{\phi, \mathrm{FB}}\left(\left[\begin{array}{c}
\phi \\
\dot{\phi}
\end{array}\right]\right)=Q_{\phi, \mathrm{FB}}\left(\left(I+t_{\mathrm{f}} N\right)\left[\begin{array}{l}
\phi \\
\dot{\phi}
\end{array}\right]\right) .
$$

In summary, despite the flight destabilization, the deadbeat nature of the stance stabilization guarantees stability in the return map sense. We list this result in Table 4.
Though this discussion of feedback stability is for the strongly coupled plant (57), we employ this controller on the physical platform Minitaur, and empirically find that even in the "weakly coupled" regime $(1-\kappa \approx 0)$ it is able to overcome preflexive bounding stability and exhibit a pronking gait (Sec. 4.4.2).

\section{Numerical and empirical results}

In this section we present all our numerical and empirical results, beginning with simulation work to test the efficacy of our models and assumptions (Sec. 4.1), and also data from a physical platform: Ghost Robotics' Minitaur (2016), a direct-drive quadrupedal robot introduced in Kenneally et al. (2016). Each of Minitaur's legs has two actuated degrees of freedom capable of independently controlling toe extension and hip flexion, with no ab/adduction joints and no passively compliant elements. Minitaur is equipped with encoders on each of its motors, as well as an inertial measurement unit (IMU). No exteroceptive sensing is required for the experiments reported here. The computationally simple controllers (leg extension controller (7) as well as other controllers mentioned below) are implemented on a STM32F303 microcontroller running at $72 \mathrm{MHz}$. The control parameters appearing in (7)-(10) used in the numerical simulation (and in correspondence with our physical platform of Fig. $1 \mathbf{D}$ as shown in Fig. 4) are: $m_{b}=5 \mathrm{~kg}, \omega=51.6 \mathrm{rad} / \mathrm{s}, \rho=0.2 \mathrm{~m}, \beta=4 \mathrm{~N} /(\mathrm{m} / \mathrm{s}) / \mathrm{kg}$, $d=0.2 \mathrm{~m}, \kappa=0.75, k_{a}=7.4 \mathrm{~N}$ (though these parameters are modified in the case of parametric sweeps).

In Sec. 4.2 we provide details of our implementation for all the gaits demonstrated on the physical platform, and empirical results in Sec. 4.3 onward. All of these experiments are also included in the video attachment.

\subsection{Simulation results}

In this subsection, we use numerical simulation (all created using MATLAB R2016a with ode45) to, first, test the efficacy of our slot hopper model and justify the various assumptions that were made for our analyses (Table 2) in Sec. 4.1.1-4.1.4. In Sec. 4.1.5, we use numerical study to get a global view of the closed-loop behavior when subject to a $\kappa$-sweep (13). An exhaustive $\kappa$-sweep would be impractical with the physical platform, though we present empirical results at two interesting values as suggested by our analysis (Fig. 18).

4.1.1 2DOF slot-hopper as a model for Minitaur In this paper we posit the 2DOF slot hopper model (Sec. 2.2) as a template-analytically tractable and effectively representative of the locomotion tasks of interest Full and Koditschek (1999)_to be anchored in a physical platform possessing far greater dynamical complexity. Even assuming massless limbs, and considering operation in only 
Table 4. Empirical extensions via template composition. Parallel composition of the new slot-hopper template with the existing SLIP template De and Koditschek (2015a) can be directly embedded into the Minitaur dynamics to achieve stable running and leaping, while relying on the analytically supported vertical energization and coordination strategies in Table 3.

\begin{tabular}{|c|c|c|}
\hline Regime & Controller(s) composed & Empirical demonstration \\
\hline & $\begin{array}{l}\text { Stepping fore-aft controller (63), yaw } \\
\text { controller }(65)\end{array}$ & $\begin{array}{l}\text { Bounding at commanded velocities up to } 5 \text { body lengths/second } \\
\text { (Fig. 15) }\end{array}$ \\
\hline "WC" & $\begin{array}{l}\text { Increased vertical gain } k_{a}(8) \text { for a single } \\
\text { stride }\end{array}$ & $\begin{array}{l}\text { Leaping from and returning to steady state bounding/pronking } \\
\text { (Fig. 16, Fig. 19) }\end{array}$ \\
\hline
\end{tabular}
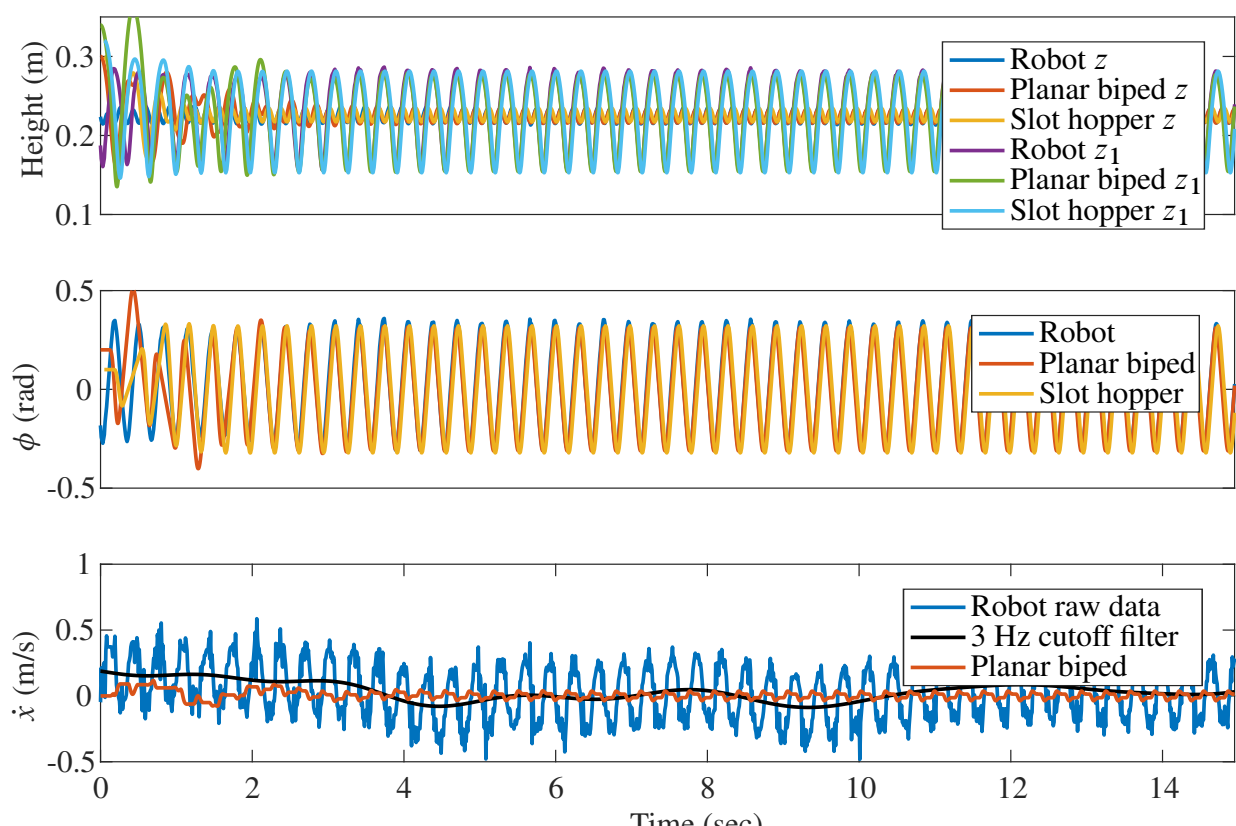
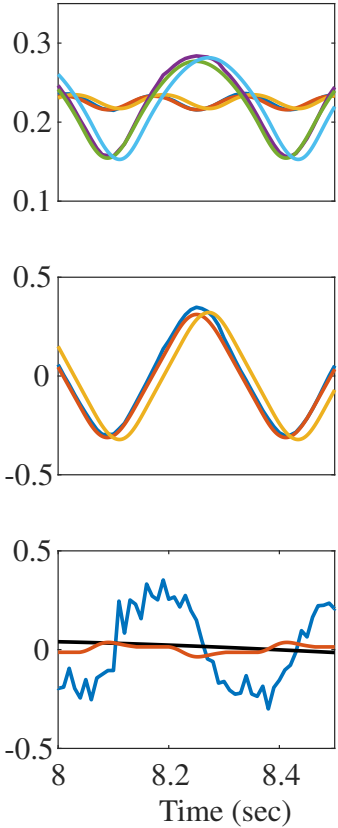

Time (sec)

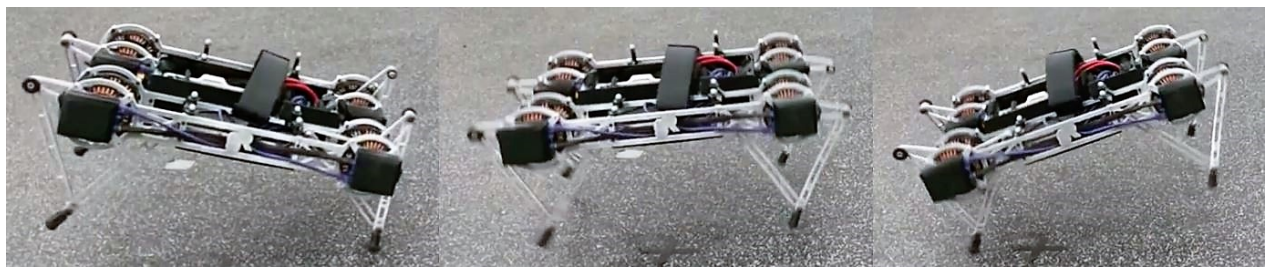

Figure 4. Projecting Minitaur and sagittal plane biped trajectories onto the slot hopper DOF. Top: A comparison of empirical data from Minitaur bounding in place (Fig. 1C) with simulations of a high-fidelity sagittal biped model (Sec. 4.1.1, Fig. 1B) and the more abstracted slot hopper model (Sec. 2.2, Fig. 1A). Note that the $x$ DOF only appears in models B-C, and its velocity is displayed here (third plot of traces) for a full account of the physical machine's behavior as it bounds in place (see Sec. 4.1.1). We provide a "zoomed-in" version on the right so that some of the almost-overlapping traces are visible (e.g. the $z$ coordinate in the first row is almost overlapped by the red/yellow traces). Bottom: Snapshots of WC-Minitaur bounding in place (taken at rear stance, aerial, and front stance) from this experiment.

the sagittal plane, a physically realistic Lagrangian model requires working with at least 5DOF kinematics, suggesting the sagittal plane biped (Fig. 1B) as an intermdiate model.

In this section, we present plots comparing the template and appropriately projected numerical and empirical body trajectories that establish: (i) the accuracy of a linearized approximation (Assumption 1) to that template; (ii) the precision with which the sagittal plane biped model (Fig. 1B) anchors that template; and (iii) initial empirical data suggesting the efficacy of that intermediate model as a description of the sagittal plane behavior of the Minitaur robot (Fig. 1C) at steady state. We reserve for Sec. 4.3 a more extensive empirical study detailing the utility of the template model's preflexive and feedback-based stability analyses for commanding and executing useful running behavior in the physical machine. 

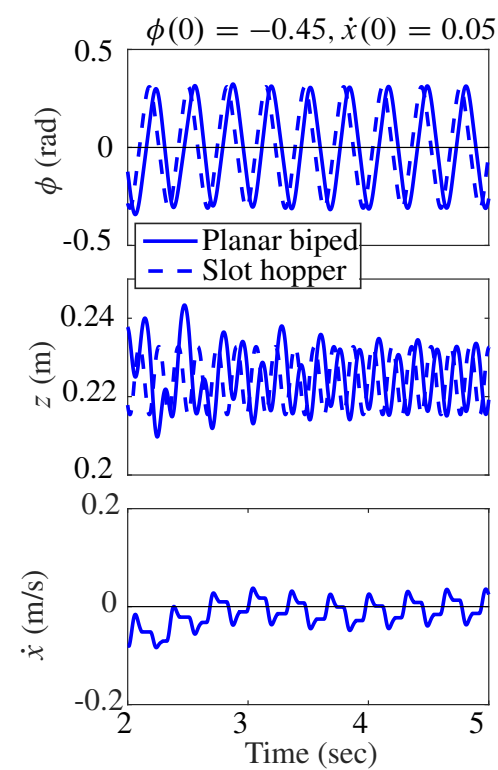
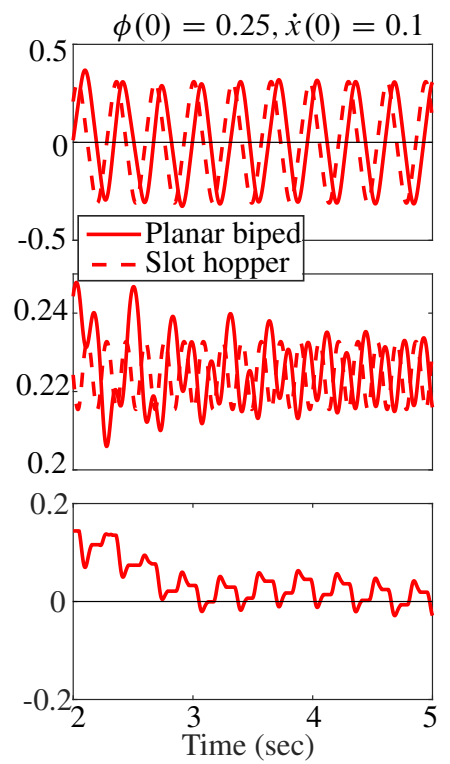
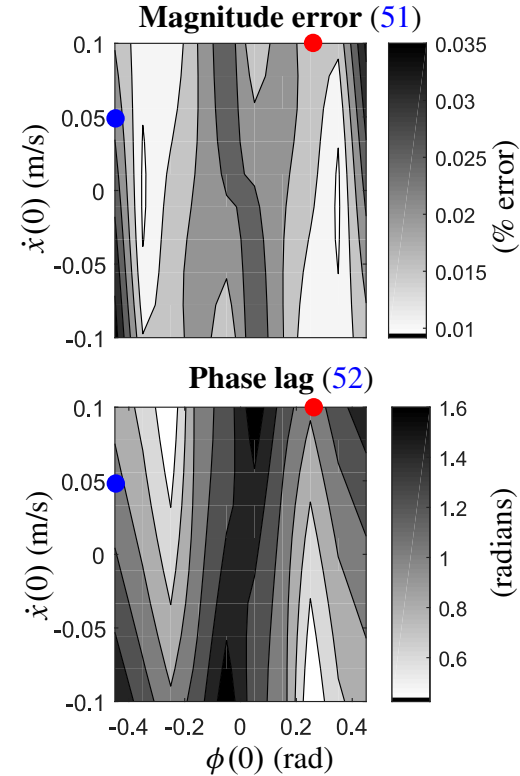

Figure 5. 2DOF slot-hopper as a template anchored by the Sagittal Plane biped. Numerical comparison of steady state behavior in the sagittal biped (Fig. 1B, Sec. 4.1.1) and in the slot-hopper (Fig. 1A, Sec. 2.2) models presenting error between the corresponding state trajectories from a variety of initial conditions. Left: time trajectories from two different initial conditions in the two columns. Note that the last traces on the left are non-existent for slot-hopper and only show that the sagittal plane biped settles down to zero-net horizontal velocity. Right: a frequency domain comparison of the resulting $\phi$ and $z$ oscillations obtained from 100 initial conditions (10 along each axis; see Sec. 4.1.1 for details). Even though the phase lag can be quite large, it converges to a constant which is generally small (as shown in the right hand sweep, and which, in any case, does not diminish the qualitative match), and the error in oscillation magnitude is bounded above by $3.5 \%$ (as discussed in Sec. 4.1.1). With the exception of the added $x$-DOF, the primary distinction between these two models is our Assumption 1; the qualitative similarity in the limiting behavior of the two models thus justifies the assumption.

This intermediate numerical model of the anchoring sagittal plane system (Fig. 1B) can be derived using the selfmanipulation modeling paradigm of Johnson et al. (2016). As shown in Fig. 1 (third column), the body is allotted 3 DOF's, and each leg is modeled as a massless revoluteprismatic joint, suggesting $\mathbf{q}:=\left[\theta_{1}, r_{1}, \theta_{2}, r_{2}, x, z, \phi\right]^{T}$, and the kinetic and potential energies are respectively $T(\mathbf{q}, \dot{\mathbf{q}}):=\frac{1}{2} m_{b}\left(\dot{x}^{2}+\dot{z}^{2}\right)+\frac{1}{2} i_{b} \dot{\phi}^{2}$, and $V(\mathbf{q}):=m_{b} g z$. Additionally, we consider two possible active contacts at the massless toes,

$$
\mathbf{a}_{i}(\mathbf{q}):=\left[\begin{array}{l}
x \\
z
\end{array}\right]+(-1)^{i-1} d\left[\begin{array}{c}
\cos \phi \\
-\sin \phi
\end{array}\right]+r_{i}\left[\begin{array}{c}
-\sin \left(\theta_{i}-\phi\right) \\
\cos \left(\theta_{i}-\phi\right)
\end{array}\right]
$$

and depending on which contacts are active, one or both constraints are activated, and appear as rows in $\mathbf{a}(\mathbf{q})$. We omit a full hybrid system description of this model, but we follow the modeling procedure of Johnson et al. (2016).

In Fig. 4, we plot data from Minitaur and data from both the sagittal biped simulation and the slot hopper simulation. Since the body inertia $i_{b}$ is not known accurately for Minitaur, we tuned that parameter from the simulation; $\kappa=0.77$ proved to be a good estimate. In the last row, the speed of the robot from raw motion capture data is plotted alongside a filtered version (obtained by truncating the Fourier transform at $3 \mathrm{~Hz}$-approximately the stride rate of bounding Minitaur). We believe that the speed oscillations are caused in part because of the motion capture rigid body CoM being located vertically above the actual CoM, and also because the body speed $\dot{x}$ does indeed oscillate in a manner that averages out over a stride due to imperfect tuning of the nominal leg angles. The leg angles and foreaft behavior are out of the analytical scope of this paper (though we describe our implementation in Sec. A.5.1).

The sagittal biped simulation's speed is also plotted, but note that the slot hopper model does not even have an $x$ degree of freedom. The fore-aft energy can be considered a "perturbation" to the slot hopper model we have analyzed here, and the persistence of the expected vertical oscillations and phase relationships testifies further to the robustness of the analytical results.

All in all, Fig. 4 indicates that at least for bounding in place, both models provide good agreement in the $z$ and $\phi$ degrees of freedom (to each other and to empirical data), over the region of state space most pertinent to the bounding task. A comparison of the slot hopper and sagittal biped models over a much larger region of state space (more trials than is practical to run on the physical platform) is shown in Fig. 5. 


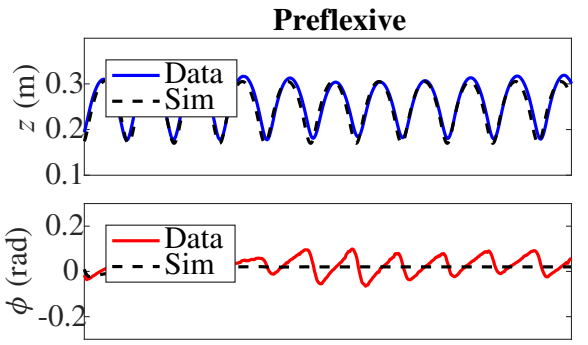

Feedback phase control
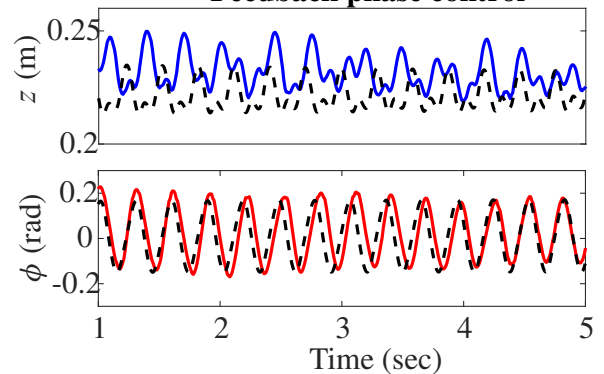

Figure 6. Comparison of slot hopper simulation to $S C$-Minitaur. The empirical data (in contrast to the numerically computed dotted traces) for this plot is from Fig. 12 and Fig. 13, and we have now used the value of $\kappa$ in (55). Note the different $z$-scales in the two sets of plots. See the last paragraph of Sec. 4.1.1 for further discussion.

The left panels of Fig. 5 show time trajectories for some relevant coordinates comparing the sagittal biped simulation to the slot hopper simulation. The simulations are started with both models in aerial phase, with different initial $\phi(0), \dot{x}(0)$ conditions, but from the same $z(0)$. Note from Fig. 4 and Fig. 15 that the initial $\phi$ on touchdown for bounding seems to be within $|\phi|<0.4$ radians empirically. The range of speeds chosen was small since this simulation does not implement the fore-aft control necessary to stabilize large speeds (in Sec. A.5.1, we describe a controller used for this purpose on the physical platform).

The leftmost columns show time-trajectories of the resulting hybrid executions, and even though the two models are drastically different in dimension and complexity, the resulting behavior is a charateristic $z$ and $\phi$ oscillation in either case. The only discernible qualitative difference is a phase shift in the resulting steady-state oscillation

In the top right column of Fig. 5, we compare the resulting oscillations in the frequency domain. Suppose $\mu_{z}(\mathbf{M})$ is the $z$-oscillation magnitude at the predominant frequency, where $\mathbf{M} \in\{\mathbf{A}, \mathbf{B}\}$ (referring to the model from Fig. 1), and a similar quantity is defined for $\phi$. In Fig. 5 (top right) we plot the quantity

$$
\left\|\left[\begin{array}{l}
\mu_{\phi}(\mathbf{B}) / \mu_{\phi}(\mathbf{A}) \\
\mu_{z}(\mathbf{B}) / \mu_{z}(\mathbf{A})
\end{array}\right]-\left[\begin{array}{l}
1 \\
1
\end{array}\right]\right\|
$$

as a metric of the normalized dissimilarity in the $\phi, z$ oscillations in the two cases. Note that each entry of the vector inside the $\|\cdot\|$ above is a "\% error" in the oscillation of the $\phi$, or $z$ trace (respectively), and we plot the norm of this vector in the top right of Fig. 5. As the legend reveals, the error is less than $3.5 \%$ over the range of initial conditions considered.

In the Fig. 5 (bottom right), we compare the phase offset of the resulting $\phi$-oscillations,

$$
\angle_{\phi}(\mathbf{B})-\angle_{\phi}(\mathbf{A})
$$

showing a larger range of phase lags between the two simulations (generally more strongly related to $\phi(0)$ than $\dot{x}(0)$ ). These discrepancies in steady state phase as a function of initial condition don't affect qualitative behavior on the limit cycles, hence, the stabilizing effects of body and feedback parameters proven mathematically for the slothopper (Sec. 3.2-3.3) achieve correspondingly stabilizing results in the qualitative behavior of the sagittal biped and physical robot as we will detail in the next section.

In addition to the numerical and empirical comparisons in Fig. 4-5, we include in Fig. 6 another comparison of the slot hopper's preflexive and feedback-stabilized behavior at a different $\kappa$ value (55) to data from SC-Minitaur. The top two rows demonstrate preflexive (pronking) behavior, and with the same $\kappa$ value, we apply the phase controller (9) to both simulation and physical platform, and plot the results in the bottom rows (bounding in both cases). The $\phi$-traces in the top row, and the $z$-traces in the bottom row both exhibit small amplitude variations, where various perturbation sources in the physical world cause apparent discrepancies between physical data and simulation. However, there is a very close match in the more pronounced $z$, and $\phi-$ oscillations that are observed in the top and bottom rows respectively between the simulation and the empirical data. This provides more evidence that the template and the analytical conclusions garnered from Sec. 3.3 and the pronking analysis in De et al. (2018) are representative of Minitaur's behavior in the real world.

4.1.2 Near-simultaneous transitions As the corresponding section of De et al. (2018) describes, an assumption on active toe extension control is required for the application of the single-mode hybrid averaging theory to our pronking analyses in De et al. (2018) and Sec. 3.3 (to ensure simultaneous transitions between aerial and double stance modes as intuitively illustrated in the lower series of (De et al. 2018, Fig. 2)). However, in practice, the resulting hybrid executions of the four-mode system (allowing for single stance) are quite similar.

In Fig. 7, we compare simulated executions of the slot hopper model (Sec. 2.2) from three different initial $\phi(0)$ conditions $(z(0)=0.4 \mathrm{~m}$ in each case), with executions of a version of this model modified as follows. In the 


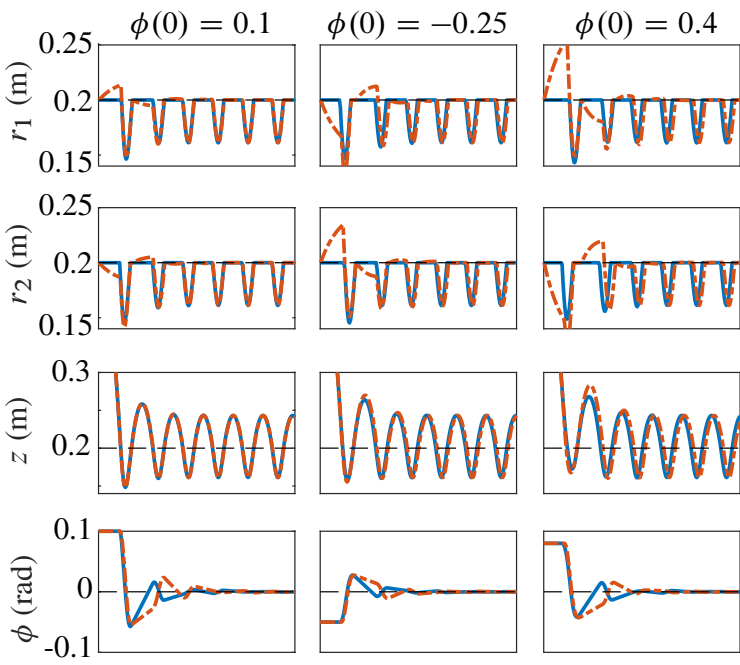

Figure 7. Qualitative effect of active toe extension for simultaneous transitions. Simulation runs of the slot hopper (Sec. 2.2, Fig. 1A) testing the simultaneous transition assumption (third row of Table 2) with some plots of executions from different initial conditions (in columns). In the traces with dashed lines, active toe extension control in aerial phase (see Sec. 4.1.2) is enabled, whereas for the solid lines it is disabled. The resulting qualitative behavior is almost identical in either case.

aerial phase, we apply the following acceleration to the toe (though the mass is non zero)

$$
\ddot{r}_{i}:=k_{p}\left(\rho+(-1)^{i-1} \phi d-r_{i}\right)-k_{d} \dot{r}_{i},
$$

for $i \in \mathcal{J}$, where $r_{i}$ is the extension of the toe in flight. Though the model assumes massless toes, this imposed feedback results in simultaneous touchdown as long as $|\phi|<\pi / 2$. In Fig. 7, the solid lines correspond to executions going through all 4 hybrid modes, and the broken lines are with simultaneous touchdown.

As expected, the larger $|\phi(0)|$ results in the most disparate behavior ${ }^{11}$, but in all cases, the asymptotic resulting behavior is identical, with small phase shifts in the $z$-oscillations. This supports our analytical assumption of simultaneous transitions (Table 2) in both the prior paper and Sec. 3.3, even though we do not enforce it on the physical platform (Sec. 4.2.3). Still, as referenced from Table 2, the relative frequency of single stance periods observed in pronking trials is quite low $(<7 \%)$.

4.1.3 Robustness to parametric inaccuracy The simple form our controller takes (7), (8) is relatively robust to parametric inaccuracy. In Fig. 8, we numerically explore the results of a mismatch in the two parameters $\alpha_{i}$ in (18) of Sec. 2.2. In the wide range of parameters shown in the bottom row $\left(g \in[-10,40] \mathrm{m} / \mathrm{s}^{2}\right.$ and one order of magnitude of variation in the assumed body mass $\left.m_{b}\right)^{12}$, the qualitative behavior still displays a stable limit cycle
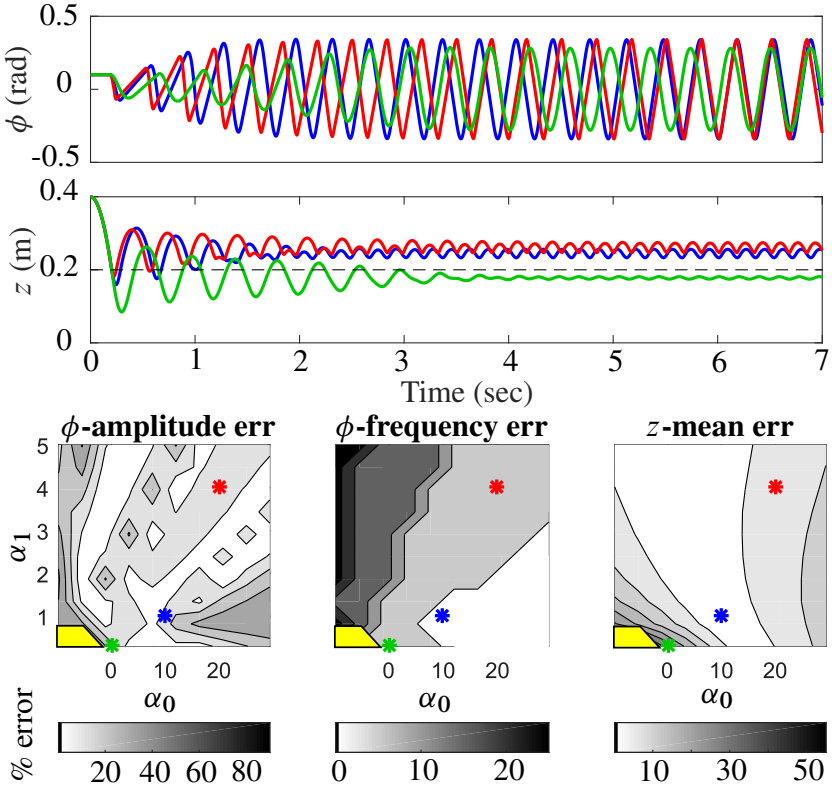

Figure 8. Robustness to parametric inaccuracy. Simulation of the slot hopper model (Fig. 1A) testing the effect of poorly chosen physical mass and inertial matching parameters, $\alpha_{i}$ in Sec. 2.2. Despite the large and varied discrepancies between assumed (applied in the control (18)) and actual $\alpha_{i}$ captured in the bottom plot, there is a stable limit cycle everywhere except for a region contained within the yellow polygon. The colors in the top plot correspond to the applied parameters designated by the " $*$ " in the bottom row. The "correct" parameters (Table 3 ) are at the blue "*" (i.e. there is no parameter mismatch); we chose the green "*" to be deliberately close to the yellow "failure region" (the lowered energy results in almost no aerial phase, causing the $z$-oscillations to be deminished; cf. Sec. 4.1.3), and the red " $*$ " in a disparate region of the parameter space. The percentage errors in the last row measure the relative discrepancy between the intended response to "correct" (blue) control parameters vs. that achieved by the actually applied parameters as described in (53) and the text following.

for almost ${ }^{13}$ the entire region. We chose $\kappa=0.9$ for each simulation in this subsubsection.

The top rows of Fig. 8 plot executions from three different parameter sets (shown in the bottom row with a "* $*$ " corresponding to the color of the solid line in the top row). The bottom row of Fig. 8 displays the discrepancy

$$
100\left(\mu_{i}(\alpha) / \mu_{i}\left(\alpha^{*}\right)-1\right)
$$

in the resulting $\phi$ and $z$ oscillations for some parameter vector $\alpha=\left[\alpha_{0}, \alpha_{1}\right]$ relative to the result with the correct parameters, $\alpha^{*}$ (blue “*” in Fig. 8), for three different "measures" $\mu_{i}$ chosen for the three columns along the bottom row of Fig. 8: the magnitude of the $\phi$-oscillation (left), frequency of the $\phi$-oscillation (center), and mean of the limiting CoM height $z$ (right). 

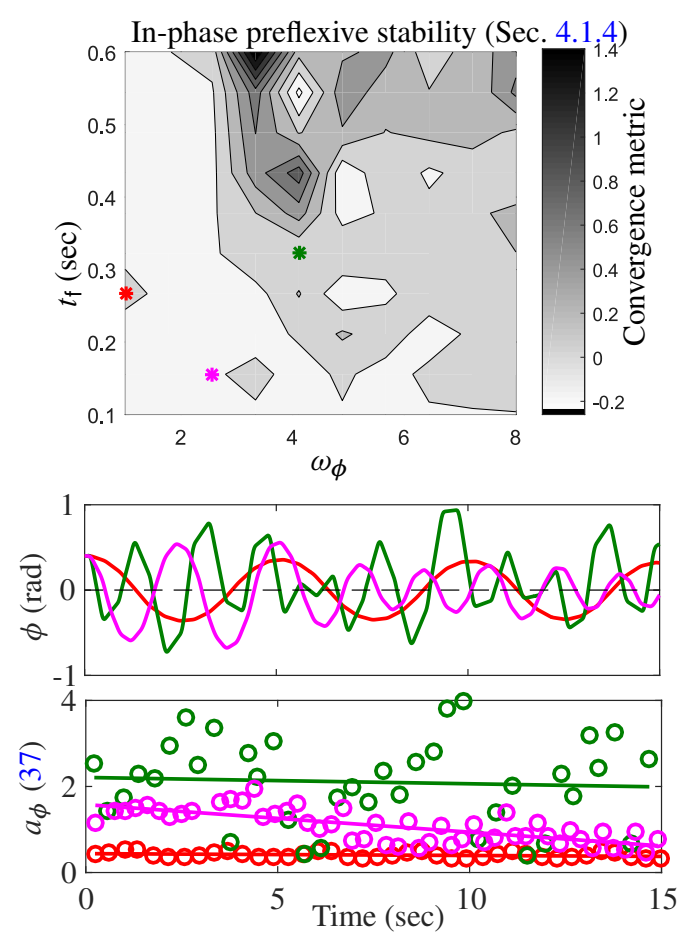

Figure 9. Strongly coupled in-phase stability vs. $\kappa$. Simulations of a slot hopper (Sec. 2.2, Fig. 1A) testing the convergence of an in-phase limit cycle as the parameters $\omega_{\phi}$ (equivalently, $\kappa$ through (40)) and the flight time $t_{\mathrm{f}}$. The "convergence metric" is an estimate (described in Sec. 4.1.4) where negative values indicate stability. We chose the pink and green parameter vectors on either side of a posited stability "boundary" supported by the findings of Sec. 3.3.6, and the red point to illustrate that the behavior for small- $\omega_{\phi}$ is convergent (albeit slow due to the small $\omega_{\phi}$ ) despite the dark patch surrounding it. The plots in the bottom row are discussed in the text.

We can see from the contours on the bottom row that excluding the yellow region ${ }^{13}$ that in each case, even large parametric mismatches in (18) result in less than $30 \%$ error as compared to the designer's intention.

4.1.4 Strongly coupled in-phase stability vs. $\kappa$ In Sec. 3.3.6, we showed that the assumption of constant flight time permits a conclusion that "high enough" $\kappa$ (consequently, "low enough" $\omega_{\phi}$ in (40)) results in preflexive pronking. In simulation, we test a range of values of $\omega_{\phi}$ across an order of magnitude to explore its interaction with a primary destabilizing influence absent this assumption. Intuitively, a greater excursion of pitch in flight should disrupt the sagittal plane gaits and, indeed, recall from the return map expression (45), the flight time $t_{\mathrm{f}}$ provides a destabilizing effect. Motivated by this, in Fig. 9, we plot a metric of stability (described below) on a $\omega_{\phi}-t_{f}^{*}$ plane. The flight time is not constant, however we provide the vertical hopper with a desired energy $a^{*}=g t_{\mathrm{f}}^{*} / 2$.
The bottom rows of Fig. 9 display both the physical $\phi$ coordinate, as well as values of $a_{\phi}$ (37) sampled at successive transitions into aerial mode (liftoff). The slope of the solid line (obtained as a least-squares degree-1 polynomial fit) in the bottom row is the "convergence metric" we use in the top row of Fig. 9. We use this unconventional metric since (as the green timeseries plot shows), in large parts of this "ungoverned" region of the parameter space, the behavior is somewhat chaotic (as apparent from the $a_{\phi}$ plot), and conventional numerical estimates of the return map eigenvalues return uninterpretable or inaccurate results. Intuitively, while in a linear system or in a small-enough basin near a fixed point, we would expect an exponential trend for state trajectories, due to the chaotic nature of the section data, in our experience a degree-1 fit proved relatively more reliable. Nonetheless, negative values of our "convergence metric" in fact indicate convergent behavior, and positive values correspond to divergence.

The plot results agree with our analytical result in Sec. 3.3.6 even without a time-invariant assumption: for each $t_{\mathrm{f}}^{*}$, there is a "low enough" $\omega_{\phi}$ that results in convergence to the in-phase limit cycle, and for smaller $t_{f}^{*}$ allows for a larger range of $\omega_{\phi}$ that result in stability. These findings are corroborated by empirical results in Sec. 4.3; Fig. 12 shows a stable empirical preflexive pronk obtained by modifying the $\kappa$ of the physical platform with no feedback-generated control signals (i.e. $w_{i} \equiv 0$ in (8)).

4.1.5 Phase locking at intermediate $\kappa$ In our analysis of the case $\kappa<1$ in Sec. 3.2.3, we showed the anti-phase limit cycle is preflexively stabilized, whereas in Sec. 3.3.6 we argued that there is a preflexive stability for the inphase limit cycle when $\kappa \gg 1$. These analyses motivate an inquiry into the behavior of the system for intermediate values of $\kappa$ (i.e., comprising a range of values around the third row of Table 3). As previously argued in Sec. 3.1, we expect the phase control to be effective in a sufficiently small neighborhood of $\kappa$ around 1; numerically and (as we show in Sec. 4.4) also empirically, we are able to explore a much larger neighborhood.

Fig. 18 shows the limiting (after $t=10$ seconds of execution) "touchdown offset",

$$
\cos (2 \pi \widetilde{s}), \text { where } \widetilde{s}:=\frac{s_{i}-s_{i-1}}{s_{i}-s_{i}^{-}}
$$

for leg $i \in \mathcal{J}, s_{i}$ is the latest touchdown time of leg $i$, and $s_{i}^{-}$ is the previous touchdown time. This calculation essentially returns $\widetilde{s}=0$ for the in-phase limit cycle, and $\widetilde{s}=1 / 2$ for an anti-phase limit cycle.

The black dots in Fig. 18 summarize simulation runs that corroborate the analytical results of this section for the slothopper model (Fig. 1A) at the left and right extremes of the plot (Sec. 3.2 and Sec. 3.3, respectively), and strongly 
Table 5. Comparison to prior virtual leg management. We refer to Raibert et al. (1989) for baseline prior practice, as it remains the most complete reference to Raibert's pioneering ideas that continue to dominate most experimental quadruped work, including our own. The analytical insights reported here afford similar behavioral results from less prescribed (event-based) compensators requiring fewer actuated degrees of freedom.

\begin{tabular}{|c|c|c|c|}
\hline Name & Raibert et al. (1989) & Our implementation & Possible benefit/discussion \\
\hline State machine & $\begin{array}{l}\text { Prescribed alternating stance/flight } \\
\text { (Fig. 6-6), cf. note } 17\end{array}$ & $\begin{array}{l}\text { Independent hips with event- } \\
\text { based (non-prescribed) mode } \\
\text { switching }\end{array}$ & $\begin{array}{l}\text { Robustly handles unexpected per- } \\
\text { turbations. } 15\end{array}$ \\
\hline $\begin{array}{l}\text { Virtual leg } \\
\text { flight control }\end{array}$ & $\begin{array}{l}\text { Identical hip-relative horiz plane } \\
\text { displacement: eqs. (6.5)-(6.6) }\end{array}$ & Same as Raibert (Sec. A.5.1) & \\
\hline $\begin{array}{l}\text { Virtual } \\
\text { leg event } \\
\text { synchronization }\end{array}$ & $\begin{array}{l}\text { Actively enforced simultaneous } \\
\text { touchdown }\end{array}$ & $\begin{array}{l}\text { Simultaneous touchdown not en- } \\
\text { forced }\end{array}$ & $\begin{array}{l}\text { Minimal qualitative effect } \\
\text { (Sec. 4.1.2), not reliant on flat } \\
\text { ground (Sec. 4.2.3) }\end{array}$ \\
\hline $\begin{array}{l}\text { Virtual leg } \\
\text { stance control }\end{array}$ & Equal axial leg forces: eq. (6.7) & $\begin{array}{l}\text { Each leg indepedently applies } \\
\text { (18) }\end{array}$ & $\begin{array}{l}\text { Shank actuators control both height } \\
\text { and roll/pitch (see below and } \\
\text { Sec. } 4.2 .3 \text { ) }\end{array}$ \\
\hline Vertical control & Increased "rest length" at bottom & Negative damping (7) & Smoothly defined through stance \\
\hline $\begin{array}{l}\text { Fore-aft speed } \\
\text { control }\end{array}$ & $\begin{array}{l}\text { Positioning flight toe at "neutral } \\
\text { point:" eqs. (6.1)-(6.2) }\end{array}$ & Same as Raibert (Sec. A.5.1) & \\
\hline $\begin{array}{l}\text { Pitch/roll } \\
\text { control }\end{array}$ & $\begin{array}{l}\text { Hip torques (flexion/extension and } \\
\text { ab/adduction): eqs. }(6.3)-(6.4)\end{array}$ & Coordination controllers (9)-(10) & $\begin{array}{l}\text { Hip actuators not needed } \\
\text { (Sec. 4.2.3) }\end{array}$ \\
\hline Yaw control & $\begin{array}{l}\text { Positioning flight toe using } \\
\text { ab/adduction: eqs. (6.9)-(6.10) }\end{array}$ & $\begin{array}{l}\text { Fore/aft hip joint torques in stance } \\
\text { (65) }\end{array}$ & $\begin{array}{l}\text { Ab/adduction joints not needed } \\
\text { (Sec. A.5.2) }\end{array}$ \\
\hline
\end{tabular}

suggest that there is a sharp "phase transition" in a tight neighborhood of $\kappa=1$ regarding which our analysis is silent absent the specific sensorimotor feedback policies analyzed in (De et al. 2018, Sec. 4.2-4.3). Looking ahead to the empirical study of the next section, the superimposed vertical green and magenta lines correspond roughly to the values of $\kappa$ exhibited by Minitaur without weights (see Sec. 4.3 reporting reflexive bounding via an anti-phase foreaft oscillation) and with weights (see Sec. 4.4 reporting preflexive pronking via an in-phase fore-aft oscillation) added to distribute the mass center. Their correspondence with this section's analysis of the slot-hopper model further attests to our hypothesis that its dynamics are anchored by preflex in the physical machine.

In contrast, we use colored dots in Fig. 18 to depict the capacity of the sensorimotor feedback policy (9) to override the preflexive coordination patterns as proven in the bounding and pronking analyses of De et al. (2018) for the case $\kappa=1$ and shown in Sec. 3.1 to apply in the "approximately decoupled" $\kappa \approx 1$ regime. Specifically, the red dots summarize the simulated results of applying (9) with $k_{d}<0$ (commanding an anti-phase limit cycle) while the blue dots correspond to the case $k_{d}>0$ (commanding an in-phase limit cycle) for the slot-hopper model (Fig. 1A), with a range of coupling values including the effectively decoupled situation of $\kappa=1$ illustrated in numerical results from De et al. (2018). While the phase control is insufficient to overcome the preflexive stability at extreme values of $\kappa$, the figure shows regions near $\kappa=1$ where the feedback control is able to stabilize near-independent hoppers to a desired limit cycle. The disruptive influence of feedback is also empirically demonstrated in the Minitaur in Sec. 4.4.

\subsection{Physical platform implementation details}

In this paper we primarily view Minitaur as a composition of two hoppers in the sagittal plane (with exceptions in Sec. 4.6). The various aspects of implementation and control are described in this subsection, and the experimental results are presented in the following subsections.

4.2.1 Template control implementation The overall implementation proceeds as follows (and is further detailed throughout this subsection): (a) a virtual leg grouping is selected; (b) physical touchdown and liftoff detection is implemented; (c) the controller (7) (and recursively, (8), and when applicable, (9) and/or (10)) is used to formulate the stance control signal; (d) this control signal is applied to the stance leg through the process described in Sec. 4.2.3; (e) to additionally move the in-place-hopping robot in the horizontal plane, the fore-aft (63) and yaw (65) controllers are applied.

In this paper we assume control authority over the radial leg force for implementation of (7). On Minitaur, this force can be controlled at a very high bandwidth using the microcontroller and drive electronics documented in De and Koditschek (2015b); Kenneally et al. (2016), and the motor encoders enable 12-bit measurements of the motor position 


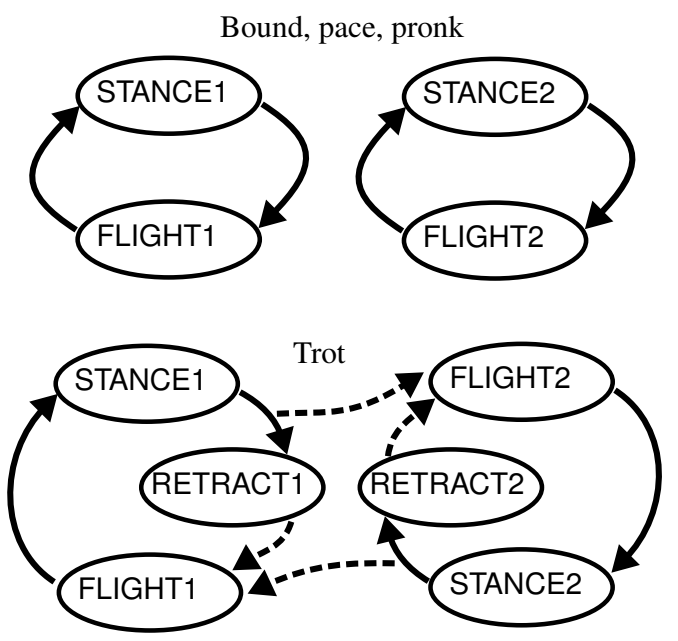

Figure 10. State machines utilized to implement the various gaits in this paper. Our work is inspired by Raibert, and this figure is meant to elicit connections to (Raibert et al. 1989, Fig. 6-6, 6-11). However, unlike Raibert's implementation (we summarize in Table 5 our departures from Raibert's implementation, and provide further details in Sec. 4.2.3 as well as Sec. A.6.2), our scheme enables bounding, pacing, and pronking with independent virtual legs and fewer actuators (only using radial leg extension), whereas Raibert's reported pace and trot results used the more aggressive sequenced coordination of (Raibert et al. 1989, Fig. 6-6), as well as two additional actuators at each hip.

and velocity. The accumulated losses from motor backEMF and bearing friction are represented in the lumped damping term in (7). Whereas there is no torsional control asserted for the in-place gaits discussed in this and the next two subsections, 4.3 and 4.4, please see Sec. 4.2.3 for an account of additional controllers introduced to coordinate other actuated and unactuated DOFs.

4.2.2 Touchdown detection A major design consideration for Minitaur was drivetrain transparency, as described in Kenneally et al. (2016). In flight, we set the toe extension using a PD controller with low gains, and on physical toetouchdown, the vertical force on the toe manifests as a deflection in the motor angles (through the well-conditioned leg Jacobian Kenneally et al. (2016)). An above-threshold deflection (corresponding exactly to an above-threshold toe force) causes the TOUCHDOWN event to be triggered. This scheme allows for touchdown detection in as little as 6 milliseconds with no additional sensors, as shown in (Kenneally and Koditschek 2017, Fig. 4, 5). Further research is underway to establish which behaviors on the Minitaur machine are crucially dependent upon this high bandwidth proprioceptive capability.

4.2.3 Virtual leg groups As (Raibert 1986, Ch. 4) describes, we use the term "virtual leg" in this paper to refer

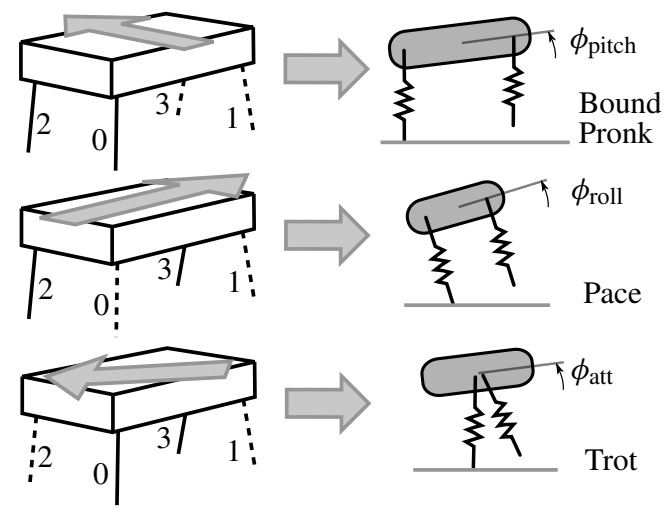

Figure 11. Virtual bipedal gaits on a quadruped. Sec. 4.2.3 discusses the feedback coordination needed for these "groupings." On the left, the solid lines are grouped together into a single virtual leg, and the dashed lines into a second one. The solid arrow connotes the "projection" of the dynamics manifesting formally as a dimension reduction of the state space considered in the analysis. The nominal leg numbering we use is also depicted in the figure, and is helpful for interpreting the empirical data in Fig. 15-21.

to a group of physical legs (on Minitaur) coordinated to operate in synchrony as per the three rules detailed in A.4. In each case, we first present our implementation, and then highlight below the similarities and differences between our implementation and that of (Raibert 1986, pg. 92). We point out here that though we use the simulation paper Murphy and Raibert (1985) and book Raibert (1986) as the main expository sources of Raibert's insights, some of the implementation details pertaining to the quadrupedal gaits demonstrated by him and his collaborators are only present in the technical report Raibert et al. (1989). We consider the latter as the definitive representation of "prior art" when juxtaposing with our own implementation, but refer to the book Raibert (1986) on occasion when the requisite descriptions are missing from the technical report. In future work we plan to explore a more sophisticated version of our intra-group control such that a "virtual leg" is formally anchored in the physical leg groups transmitting ground reaction forces to the robot body, as in Saranli and Koditschek (2003).

\subsection{Preflexive stability}

First, we leverage the preflexive stability properties analyzed in Sec. 3.2 and 3.3 to get stable limit cycles on Minitaur without any feedback coordination of the phases of the two hips. We program each of the sagittal plane legs (virtualized according to the top row of Fig. 11) as a vertical hopper, using the controller (7) with $w_{i} \equiv 0$ in (8).

4.3.1 Bounding in place The first result we obtain is that Minitaur bounds in place (Fig. 4). In that figure we compare 

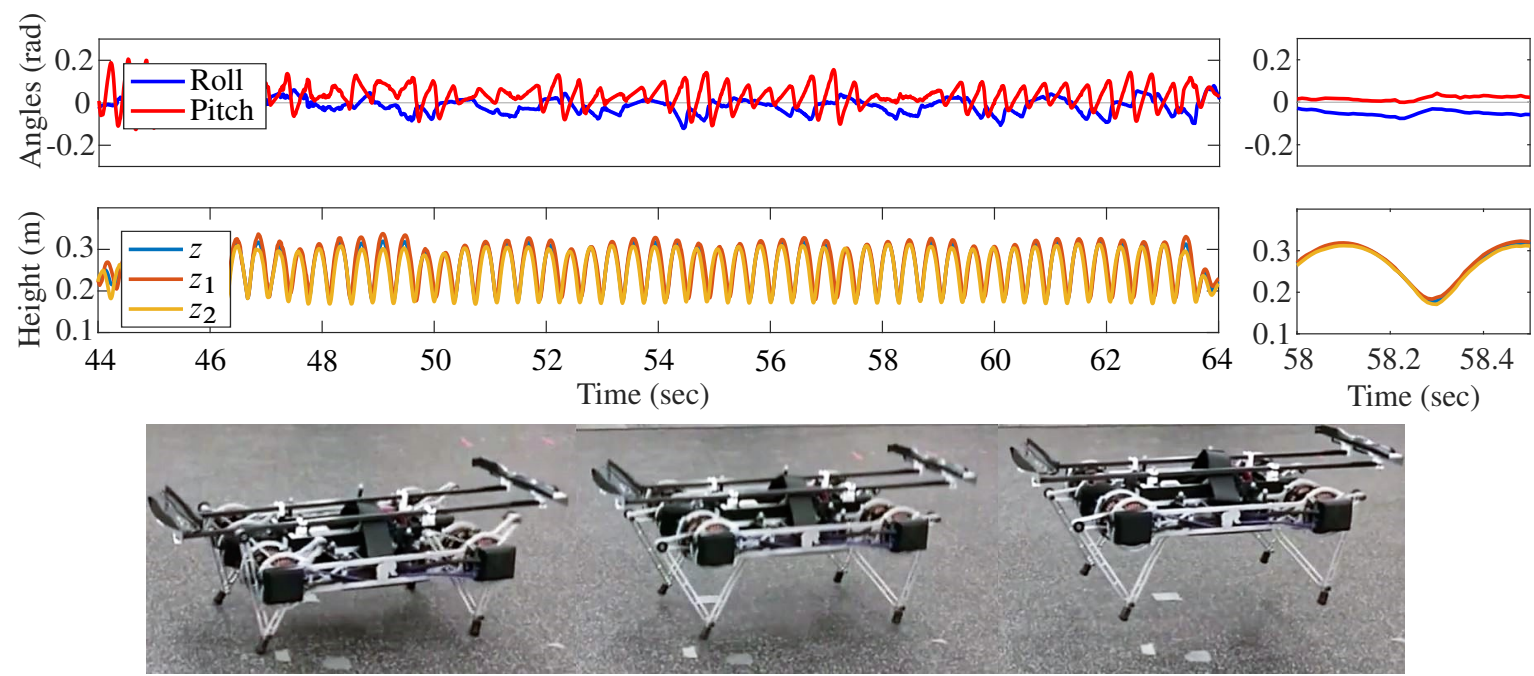

Figure 12. SC-Minitaur preflexive pronking. Top: Empirical data from Minitaur (Fig. 1C) in its "high-inertia" configuration (Sec. 4.3.2) showing stable preflexive pronking (note that the hip heights $z_{1}, z_{2}$ oscillate in phase). We compare this empirical data to our simulation models in Fig. 6. Additionally, by examining successive touchdown and liftoff times from the log file, we get statistics for the time of stance $t_{\mathrm{S}}=0.139 \pm 0.013$ seconds, and time of flight $t_{\mathrm{f}}=0.233 \pm 0.026$ seconds. The deviation in flight time is thus $11 \%$ including the initial transients, justifying our investigation into "constant flight time" in Sec. 3.3.6. Bottom: Snapshots of SC-Minitaur (taken roughly at bottom, liftoff, and apex) from this experiment, showing the newly attached $0.5 \mathrm{~kg}$ weights on either end (compared to Fig. 4) to modify $\kappa(13)$.
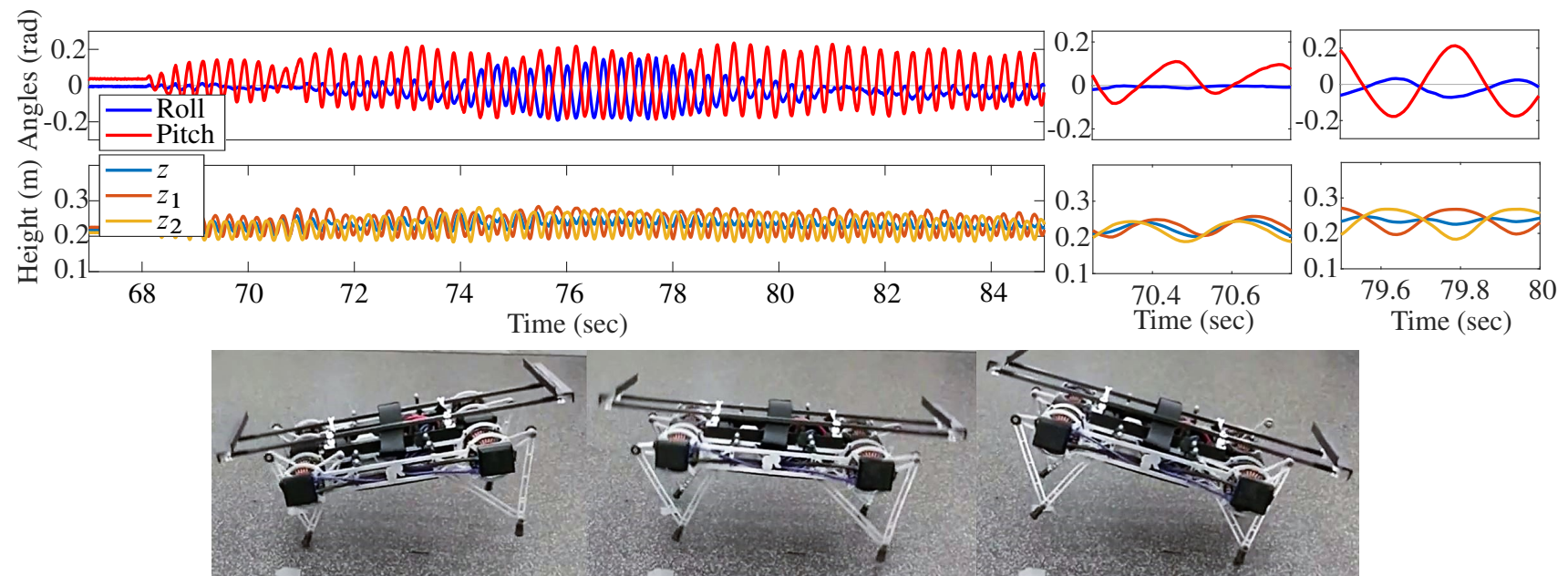

Figure 13. SC-Minitaur feedback-stabilized bounding. Top: Empirical data from Minitaur (Fig. 1C) in its "high-inertia" configuration (Sec. 4.3.2), but with feedback phase control (9), showing stable bounding (note that while in the initial transient phase (first inset), the hip heights $z_{1}, z_{2}$ oscillate without a specific relative phase, after some time they stabilize to being out of phase, and the pitch oscillations are much more pronounced). We compare this empirical data to our simulation models in Fig. 6 , and discuss in Sec. 4.4.1. Bottom: Snapshots of SC-Minitaur bounding in place (roughly at front stance, aerial, and rear stance) from the same experiment.

the resulting time-behavior of the appropriately chosen physical coordinates to simulations of the numerical models depicted in Fig. 1 , and find that $\kappa \approx 0.77$ is a good fit. This matches our expectation, since most of Minitaur's mass is concentrated at the hips (where the motors are attached), but some mass is concentrated near the center where the battery is housed. This preflexive stability is a demonstration of our analytical result in Sec. 3.2, and also shows remarkable agreement of empirical data to the simulated sagittal plane biped (Fig. 1B) and only slightly more distinguishable traces relative to the slot-hopper template (Fig. 1A).

4.3.2 Pronking in place In the next experiment, we artificially modify $\kappa$ by attaching two weights on top of Minitaur (we deem the modified model "SC-Minitaur" or 

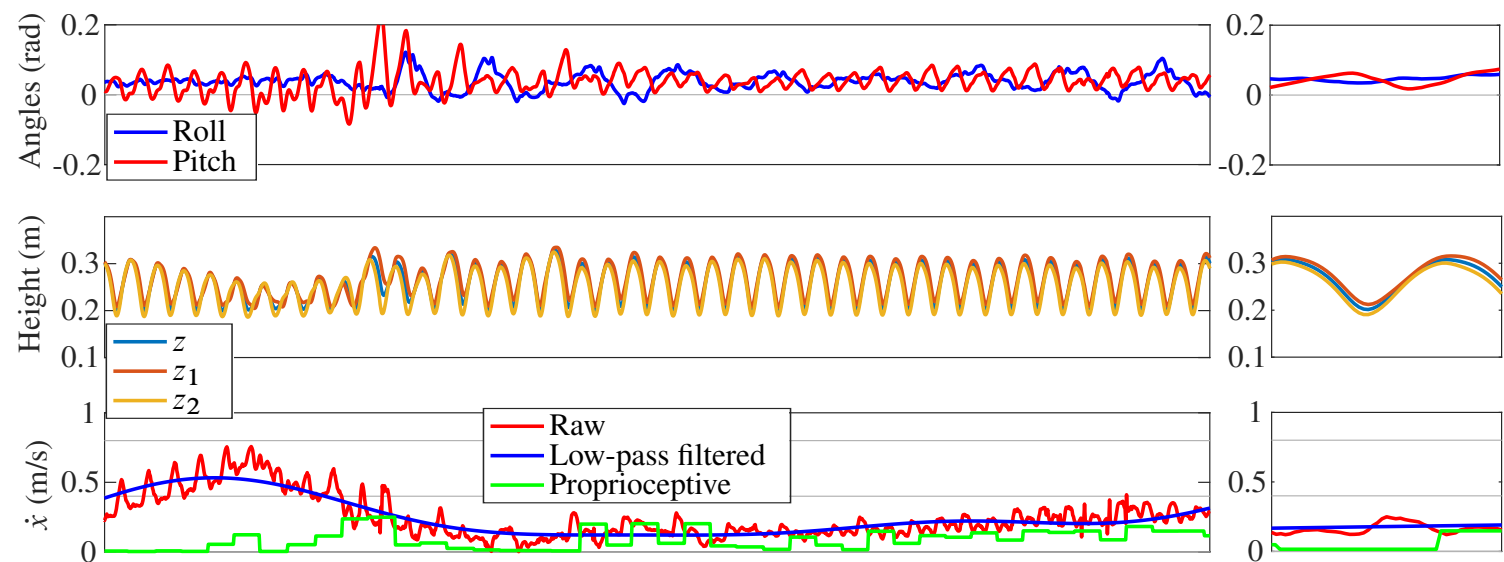

0.1
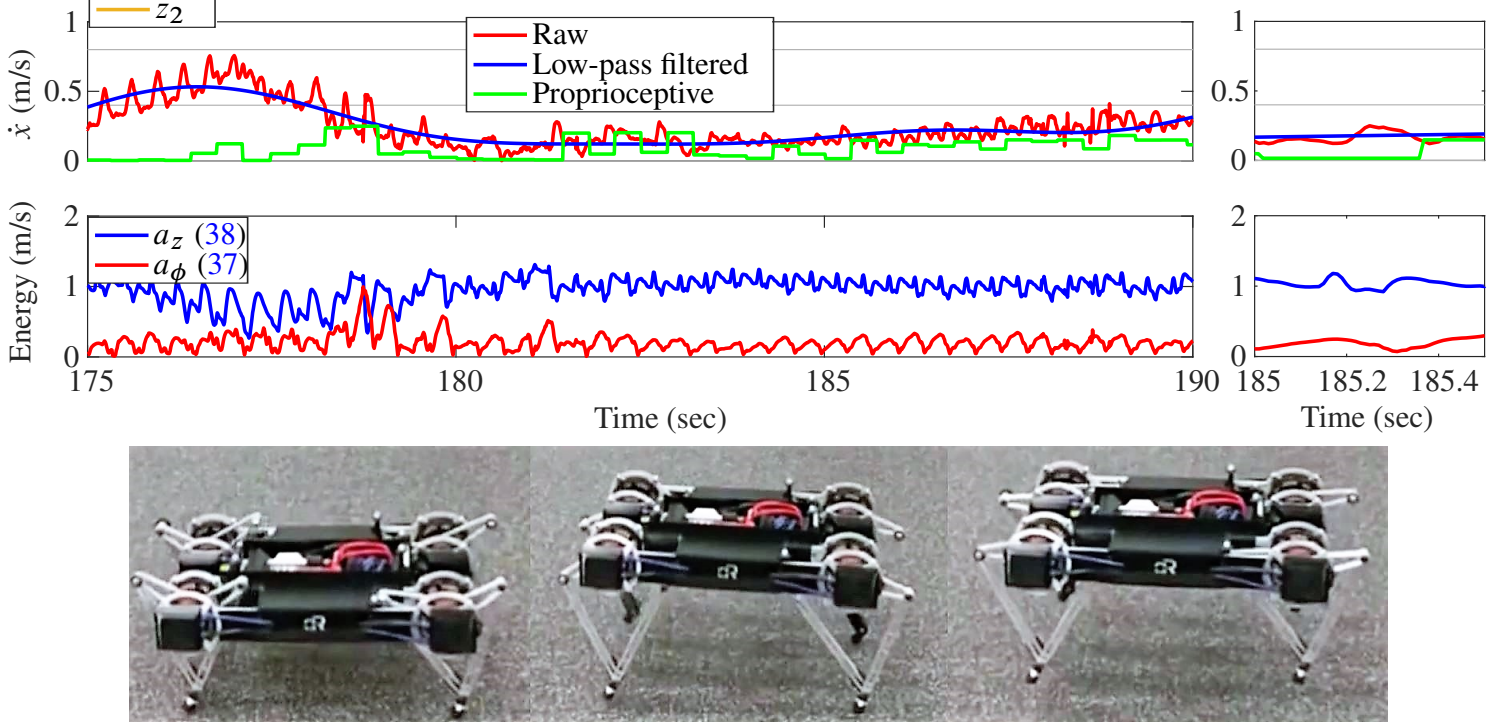

Figure 14. Minitaur feedback-stabilized pronking. Top: Empirical data from Minitaur (Fig. 1C) in its default configuration, but with feedback attitude control (10) stable pronking (note that the hip heights $z_{1}, z_{2}$ oscillate in phase). As described after (38), the "energy" coordinates have units of speed. See Sec. 4.4 for more discussion. Bottom: Snapshots of WC-Minitaur pronking (taken roughly at bottom, liftoff, and apex) from this experiment.

strongly-coupled Minitaur). These outrigger payloads have a total mass of $1 \mathrm{~kg}$, are added at a distance of $0.37 \mathrm{~m}$ from the center of mass, and primarily serve to increase the rotational inertia about the center of mass (Fig. 12, bottom). Using the parallel axis theorem, the resulting inertia is

$$
\begin{aligned}
i_{b}^{\prime}=\kappa m_{b} d^{2}+1 \cdot 0.37^{2} & =: \kappa^{\prime}\left(m_{b}+1\right) d^{2} \\
& \Longrightarrow \kappa^{\prime}=1.149
\end{aligned}
$$

using values for $m_{b}$ and $d$ mentioned in the introduction of Sec. 4 , and $\kappa$ mentioned in Sec. 4.3.1.

We find that the modified SC-Minitaur exhibits a preflexively stable pronking in place, as shown in Fig. 12. We reiterate to emphasize that the controller was not modified between Fig. 4 and Fig. 12, only the physical parameters of the body. This preflexive stability is an empirical demonstration of our stability analysis in Sec. 3.3.

Qualitatively, we observe that preflexively, the legs $z_{i}$ are in phase in SC-Minitaur Fig. 12, but out of phase in unloaded Minitaur (Fig. 4 and Fig. 15). We did not include $z_{2}$ in the top row of Fig. 4 for clarity, but the different asymptotic relative phases can be seen by comparing the zoomed-in panels in Fig. 4 to Fig. 12; specifically, the reader should compare the relative phases of the red and yellow traces. This empirical outcome is expected from our analytical results of Sec. 3.2 (preflexive bounding) and Sec. 3.3 (preflexive pronking), and the simulation results generated from our slot hopper model in Fig. 18. Additionally, the pitch shows a pronounced oscillation in both Fig. 4 and Fig. 15 (instances of bounding), but the magnitude of the pitch is very small in Fig. 12 (instance of pronking).

\subsection{Feedback synchronization}

Simulations in the companion paper De et al. (2018) showed that the coordination controllers (9)-(10) can establish either bounding or pronking limit cycles in the decoupled slot hopper. In this paper, we show that the same controllers can reinforce or disturb the slot hopper's preflexive stability (proved in Sec. 3) when applied to a slot hopper simulation (Fig. 18). In this subsection, we now apply the feedback phase control ideas of Sec. 2.3 to attempt to disrupt the limit cycles of Sec. 4.3. 


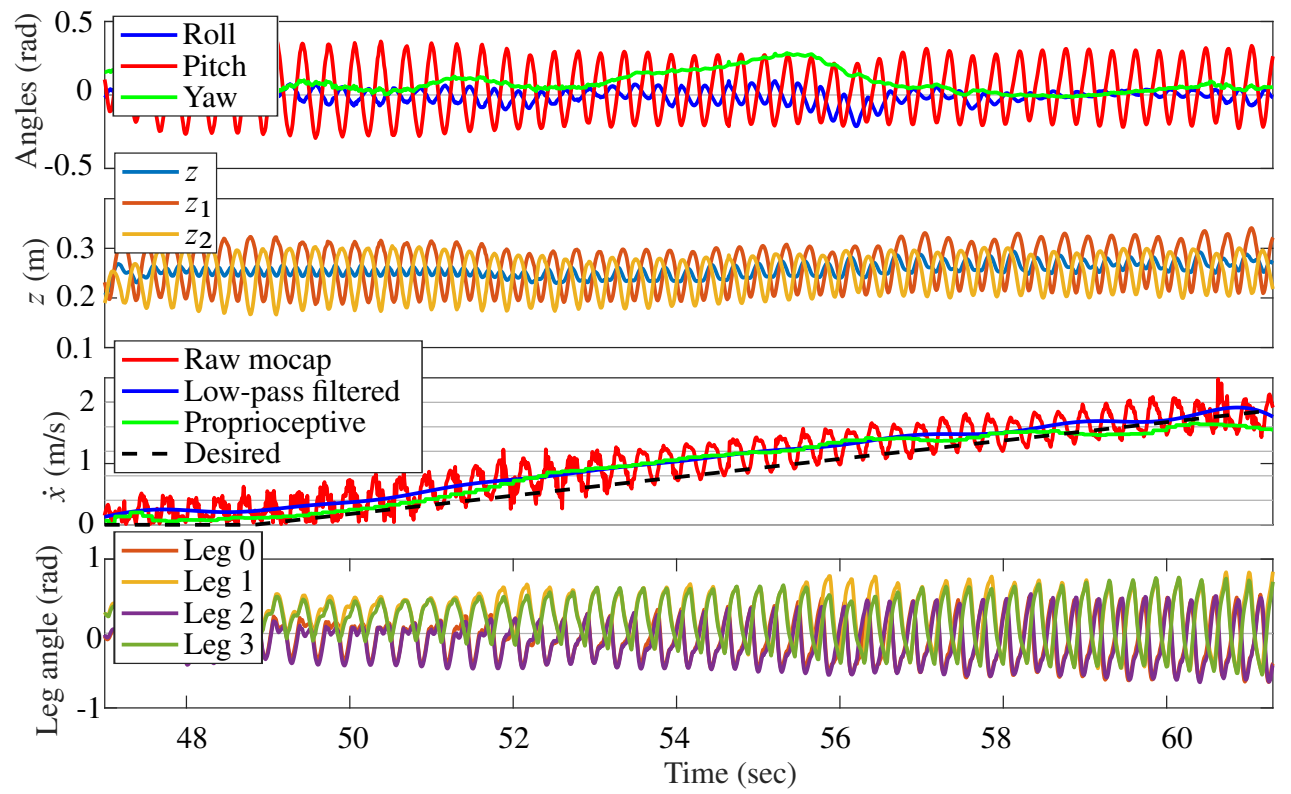

Figure 15. Minitaur preflexive bounding. Empirical data from Minitaur (Fig. 1C) exhibiting bounding with preflexive coordination (pitch) control (Sec. 3.2), feedback roll control (10) (roll angle is also displayed in the first row), moving at a range of commanded forward speeds (Sec. A.5.1) up to 4.8 body lengths/sec, as well as yaw rates (Sec. A.5.2), controlled using a parallel composition of decoupled controllers. See the last paragraph of Sec. A.5.1 for discussion. Lastly, numerical post-processing of this data reveals that despite the strong fore-aft and yaw perturbations in this trial, the stance duration is $109 \pm 7.5 \mathrm{~ms}$ (mean and standard deviation): a 6.5\% deviation. As in Raibert (1986), this motivates our constant stance duration assumption in Table 2.

We first point out that the two Minitaur configurations listed in Sec. 4.3 are indicated with the vertical green (WCMinitaur, Fig. 1C) and magenta (SC-Minitaur, Fig. 12) lines in Fig. 18. Our empirical trials reveal that the phase control (9) is able to overcome the preflexive stabilization in the magenta configuration (see Sec. 4.4.1 below) to disrupt the mechanically entrained pronking in favor of the "commanded" bounding gait. On the other hand, more aggressive feedback coordination (10) is required to overcome the preflexive stability in the green configuration (see Sec. 4.4.2 below) to disrupt the mechanically entrained bounding in favor of the "commanded" pronking gait. ${ }^{16} \mathrm{We}$ remind the reader that these experiments are recorded in the video attachment as well.

\subsubsection{Using phase control to disrupt preflexive pronking} and impose bounding We implement the feedback controller (9) on SC-Minitaur, and plot the results in Fig. 13. Compared to the results with the preflexive control only (Fig. 12), a pitch oscillation can be observed, and furthermore, the second row reveals an alternating front/rear touchdown in $z_{1}$ and $z_{2}$, indicating that the anti-phase limit cycle characteristic of bounding has been stabilized. In the region $t \in[74,79]$ seconds of Fig. 13, the robot rolls in an underdamped fashion due to the attitude controller applied in the frontal plane (61) not being tuned ideally. The sagittal plane behavior is mostly unaffected by this rolling.

We can conclude that the $\kappa$ of SC-Minitaur (magenta line in Fig. 18) allows the phase control (9) to overcome the preflexive control analyzed in Sec. 3.3. We display this data point in Fig. 18 as well.

An additional example of the application of phase control can be found in the application to the pacing gait presented in Sec. A.6.1. However, note that this application is beyond the analytical scope of the present paper, since Minitaur does not possess hip ab/adduction joints. Consequently, the body-leg angle is rigidly set at $\pi / 2$, and (a) the legs cannot be positioned vertically with respect to the ground, and (b) ground reaction forces are not always directed vertically as they are in the slot hopper.

4.4.2 Using attitude control to disrupt preflexive bounding and impose pronking The phase controller (9) is not successful in overcoming the preflexive control in WC-Minitaur (green line in Fig. 18), indicating that the preflexive controller is "stronger." We apply the more aggressive attitude-servoing controller (10) in order to attempt to stabilize an in-phase limit cycle (pronking).

The results in Fig. 14 show an in-phase pattern for $z_{1}, z_{2}$, indicating stability of the in-phase limit cycle. Moreover, the magnitude of the body pitch has been reduced to approximately 0.15 radians, compared to the much larger 


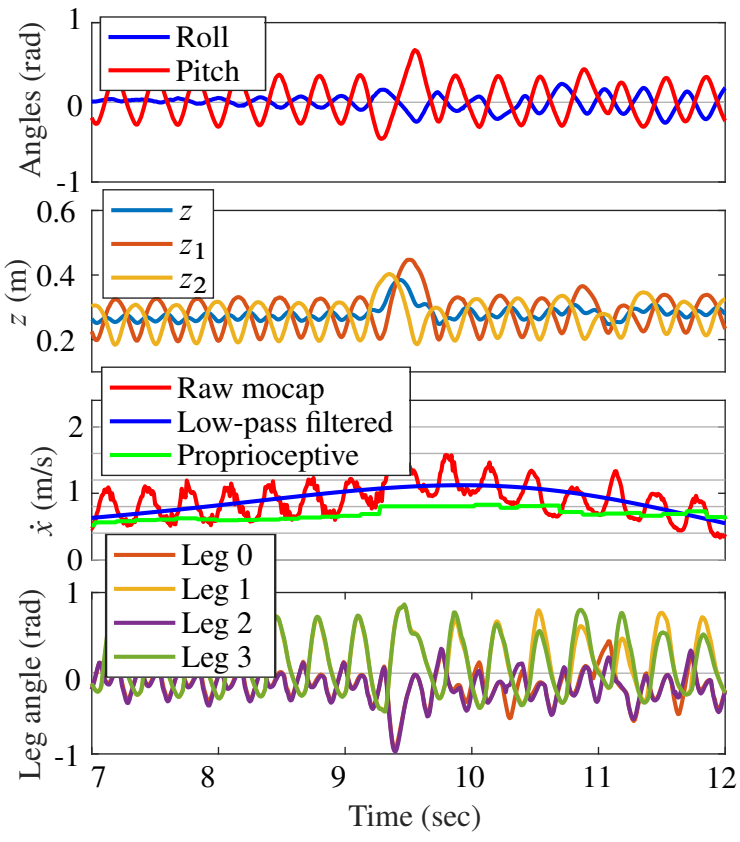

Figure 16. Minitaur bounding leap. Empirical data from Minitaur (Fig. 1C) exhibiting bounding (Sec. A.5.1), with a discrete "leap" command send at $t=9$ seconds (higher desired vertical energy for a single stride at both hips) resulting in a vertical displacement of the center of mass while bounding.

pitch oscillations seen in the bounding trial in Fig. 4. We display this data point in Fig. 18 as well.

\subsection{Parallel template compositions for translation and rotation of the COM}

Raibert (1986) pioneered a kind of empirical compositiondecoupled controllers, each designed to stabilize an isolated 1DOF "template plant," which, when utilized in tandem, empirically stabilize a high-DOF body. The empirical success betrays the difficulty in obtaining provable guarantees, however: coupling in mechanical systems can move energy between the different compartments, either stabilizing or destabilizing the template plant.

In Murphy and Raibert (1985), the following quote appears shortly after the one we have used in Sec. 1: "there was a limit on forward velocity. As the model bounded faster, the height of centre of gravity of the body decreased along with the magnitude of its angular oscillation. These two factors reduced the ground clearance of the foot as it swung forward. Eventually, the model stubbed its toe, tripped and fell." This anecdote confirms the difficulties of composing fore-aft control with the vertical energization (7) and coordination (9)-(10) discussed thus far. This motivates further analytical work to discover and prove stability of feedback-stabilization of other DOFs, but we

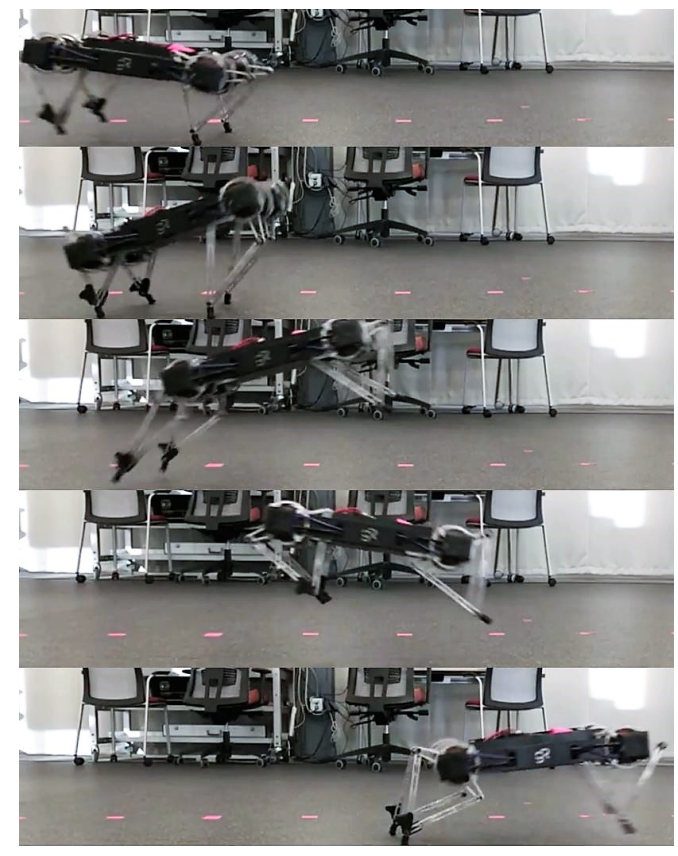

Figure 17. Snapshots of WC-Minitaur performing a bounding leap, showing that this simple strategy allows the robot to clear a gap larger than 1.5 times its body length.

restrict the scope of this paper to empirical composition of controllers. We find empirically that the simplicity of our template controllers (7)-(10) affords robustness against large amounts of coupling energy (arising from the significant cross talk injected by these erstwhile decoupled controllers) before destabilization.

Though the empirical demonstrations in this section go beyond the scope of our analytical results, they are each distinctly relevant to the topic of this paper: (a) fore-aft bounding (A.5.1) demonstrates of robustness to persistent periodic perturbations from coupling interactions with the fore-aft DOFs; (b) horizontal-plane yaw control (A.5.2) demonstrates the utility of the coordination controllers developed in this paper as well as the attitude controller used in the roll plane when subjected to aperiodic perturbations when turning; (c) leaping and return to steady motion (A.5.3) demonstrates the robustness of the (preflexive or feedback) coordination controller to large instantaneous perturbations in the relative phase of the two legs. For this subsection and the next, detailed descriptions of our experiments are in the Appendix.

\subsection{Pacing and trotting}

So far we have presented data from Minitaur bounding and pronking, but now we show how the various regimes (Table 3) of the simple 2DOF slot hopper model introduced in this paper also pertain to trotting and pacing, covering the full gamut of virtual bipedal quadruped gaits. Moving 
beyond the sagittal plane (while still only using the controllers (7), (9) and (10) as applicable), Minitaur is able to exhibit the remaining virtual bipedal gaits: pace and trot. A pictorial description of these gaits from the virtual leg viewpoint is in Fig. 11.

Our implementation of pacing and trotting on a physical machine differs from that reported in Raibert et al. (1989), in ways that we summarize below:

a) we are able to exhibit these gaits while using fewer actuators: only shank actuators in our case as compared to two actuators (fore-aft and ab/adduction) at the hip (Raibert et al. 1989, eqs. (6.3), (6.4)) due to the analytical insights from De et al. (2018) suggesting the use of the $w_{i}$ term in (8) for phase (9) or attitude (10) control;

b) the state machine in (Raibert et al. 1989, Fig. 6-6) forces a prescribed alternating stance/flight sequence, whereas our state machine (Fig. 10) bears more resemblance to Raibert's "independent" version in (Raibert et al. 1989, Fig. 6-11) which is reportedly only used for bounding and pronking. ${ }^{17}$ One advantage of using a less prescriptive state machine is that unexpected double stance (caused by unexpected ground contact on rough terrain) is handled gracefully, whereas it falls completely outside the logical jurisdiction of (Raibert et al. 1989, Fig. 6-6).

Our pacing and trotting experiments are described in A.6.1A.6.2.

\section{Conclusion}

We have presented a method for implementing all the virtual bipedal quadruped gaits on Minitaur using just shank extension actuators (for in-place bouncing) by recourse to a simple three-parameter family of controllers (7)-(10). The corresponding robustly stabilized family of behaviors admits reliable, rapid changes in set point command, for example, interspersing steady gaits with leaps up to $2 \mathrm{leg}$ lengths. Adding on hip flexion actuation, we obtain diverse and reliably robust modes of locomotion at up to 5 body lengths/second.

This family of controllers arises from insights into the slot hopper, a new template for coordination of inplace virtual bipedal gaits that exhibits two different kinds of preflexive stability as its non-dimensional inertia (13) is varied. We leveraged hybrid averaging De et al. (2018) to provide (to our knowledge) the first proofs of preflexive virtual biped bounding and pronking using this template. We have also presented extensive numerical and empirical evidence that these analytical results have strong qualitative bearing on Minitaur's behavior in the physical

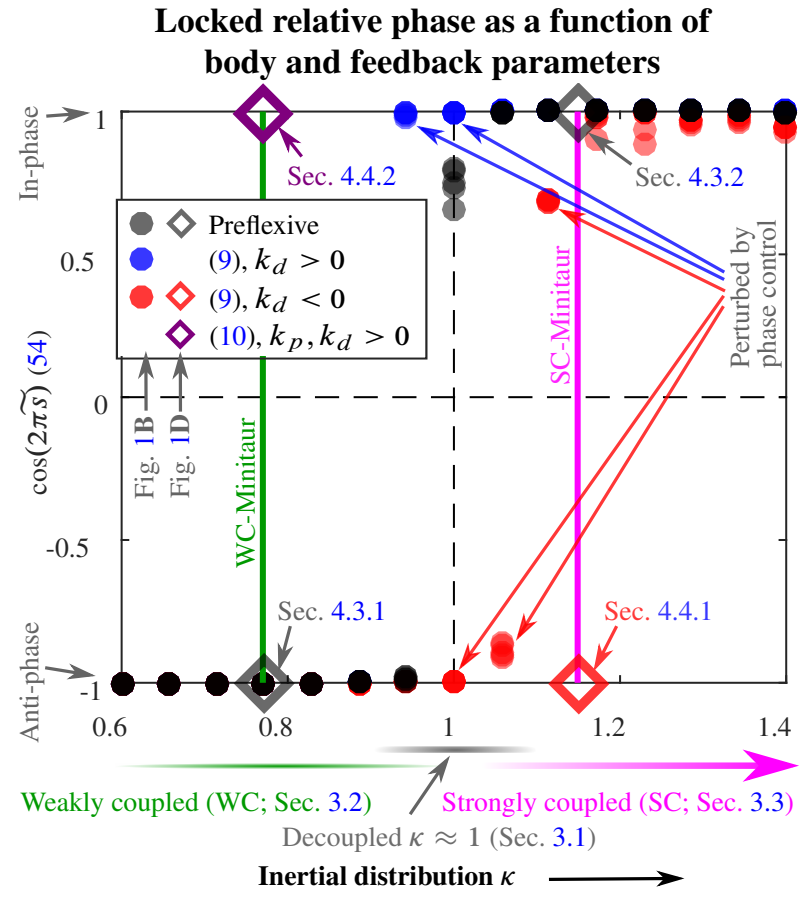

Figure 18. Phase locking as a function of body inertia and feedback gain parameters. Stable steady-state relationships between leg phases for simulation and physical runs of the models depicted in Fig. 1 in relation to the inertia parameter $\kappa$ (13). Solid round dots represent the limiting relative phase between the two legs of the slot hopper model (Sec. 2.2, Fig. 1B). Each gray translucent dot is the result of a single trial ( 5 trials are plotted at each $\kappa$ value, though in many of the trials, the dots overlap and appear as a single dot of increased opacity) which is $t_{\max }=5$ seconds long, and the phase offset is calculated at the end. The gray dots refer to a preflexive control only $\left(k_{d}=0\right.$ in (9)), whereas, in contrast, the blue and red dots contain trials with some phase control (9). The hollow diamonds are data points representing Minitaur's preflexive and feedback-stabilized gait coordination behavior in the physical world (Sec. 4.3-4.4, Fig. 1D). Please refer back to Sec. 1.2.1 for our textual summary of the major claims of the paper, now recapitulated in detail through this figure.

world. Specifically, as summarized in Fig. 18, they yield analytically formulated controllers (9)-(10) that augment / disturb preflexive stability and enable a diverse set of behaviors on the same physical body.

\subsection{Discussion}

5.1.1 Hybrid averaging for analysis of dynamical compositions The mathematical basis for our formal results-the stability of the bounding and pronking behaviors in the first two abstract models of Fig. 1rests upon the hybrid averaging tools in De et al. (2018). However, the central contribution of this paper is to demonstrate the utility of this analysis to the development 
of robust, dynamic gaits on a general purpose physical quadruped.

Analytical insight about the slot hopper gives us useful understanding about (a) conditions under which our proposed control strategies succeed, (b) indications about conditions under which they will fail to work, and lastly, (c) information about the relation of the control strategy to the morphological parameters of the robot body. ${ }^{18} \mathrm{In}$ this paper, we have provided a detailed example of the benefits of analytical insight. Murphy and Raibert (1985) and Raibert et al. (1989) observed preflexive bounding stability in their simulation and physical models; later Berkemeier (1998) provided a restricted ${ }^{19}$ explanation of this stability. We are now able to (using formal analysis) for the first time (a) explain the mechanism of this preflexive bounding stability, (b) explain a second mechanism for preflexive pronking stability, (c) explain analytically and not anecdotally the relation of the morphological "nondimensional inertia" parameter to both preflexes, and (d) use our analytical insight to not just exploit but also countermand the preflexive tendencies on our quadrupedal robot to achieve a highly robust gait stabilization feedback paradigm requiring very few tuning parameters ${ }^{1}$.

In consequence of (the long-known Holmes (2005)) nonintegrability of most two (and higher) DoF mechanical systems no procedural strategy has been developed for analyzing many 2DoF (and effectively any higher DoF) hybrid dynamical systems representative of locomotion (LLS Schmitt and Holmes (2000), SLIP with torso, etc.). By providing a principled means of approximating these generically non-integrable systems with formally "nearby" integrable provably "close enough" stand-ins, we believe our averaging approach may offer a route toward such a procedural analysis of much higher dimensional locomotion systems than has heretofore been possible, allowing us to reap the aforementioned benefits.

We have shown in the bounding analysis of Sec. 3.2 how coupling forces can cancel "on average" (e.g. the last slot of (31)) to permit dynamical composition of the two hoppers into a single bounding body without destabilization from coupling. We are encouraged by the prospect of our averaging analytical result revealing how to compose dynamical primitives for synthesizing behaviors such as the useful bounding, pronking, trotting, pacing, and leaping demonstrated here, the tailed planar hopping we have demonstrated before De and Koditschek (2015a), and many other examples in the future.

Work now underway is attempting to formalize the utility of time-reversal symmetries Altendorfer et al. (2004) in an averaging framework. Our goal is to further the anecdotal observations of (Raibert 1986, Ch. 5) about "symmetric stances," and show and generalize their utility in "averaging out" a subset of pointwise-in-time deleterious coupling forces.

\subsubsection{Multiple phases in strongly-coupled analysis Our} present framework De et al. (2018) (and indeed, classical non-hybrid dynamical averaging, for which our reference is Guckenheimer and Holmes (1990)) only accounts for a single "fast" coordinate. Our analysis of the stronglycoupled hoppers was contingent on assumption 2(a), which allowed us to decouple the $z$ and $\phi$ dynamics in (60), was crucial because otherwise the $z$ and $\phi$ dynamics oscillate in a non-phase-locked manner. Future work in applied dynamical systems to enable averaging across several loosely interacting "phase" variables may make a full coupled analysis possible.

Additionally, we required a restrictive condition on the flight time (assumption 2(b)), essentially due to the timevarying nature of the $\phi$ return map (Sec. 3.3.5. A much more useful conclusion about $\kappa$ was derived with a timeinvariant assumption in Sec. 3.3.6. We believe that a theory of "small" time-variations that allows us to use the eigenvalues of $P_{\phi}$ would make the strongly coupled stability proof not require assumption 2(b).

5.1.3 Limiting value of $\kappa \rightarrow 0$ The first row of Table 3 refers to a degenerate condition, where all the mass of the body is concentrated at its center. In this configuration, the $\phi$-DOF is degenerate (since $i_{b}=\kappa m_{b} d^{2}=0$ ). Thus, we are effectively reduced to a $1 \mathrm{DOF}$ vertical hopper without any physical relevance, since the model in Fig. 1B posseses a "body" whose orientation now cannot be controlled.

5.1.4 CoM templates for virtual bipeds In this paper, we thought of each hip as a vertical hopper: a loose "templateanchor" relation Full and Koditschek (1999) that we do not make formal here. In the future, we hope to formalize a definition of "averaged anchoring." However, without making any formal statements, we can note the following: As (21) shows, "weakly coupled" hoppers behave as if each hip is still a vertical hopper (i.e. the mechanically decoupled hoppers of De et al. (2018) are a reasonable template). The behavior of the CoM $(z)$ is revealed to be oscillatory from Fig. 5, albeit with small magnitude relative to the hips. As Fig. 15 reveals, the center of mass displays small vertical and horizontal oscillations, but over a full stride, seems to behave like a point particle in the sagittal plane.

In strongly coupled hoppers (subject to assumption 2(a)), the CoM behaves like a single vertical hopper (60). Our empirical data of the pronk (Fig. 14) and the trot (Fig. 21) reveal vertical oscillations of the CoM (suggesting a SLIPlike template), though the magnitude of vertical oscillation is much smaller in the current trot implementation.

5.1.5 Multiple shank energization strategies The shank-energization strategy we used in this paper is quite 
different from the "thrust-at-bottom" strategy utilized by Raibert (1986), but both exhibit the preflexive stability characteristics originally observed in Murphy and Raibert (1985) and analyzed in Berkemeier (1998). Though our new analytical tools allowed us to better understand its origins (Sec. 3.2-3.3), we are still far away from a theory that might encompass many alternative shank energization strategies including Raibert's; more insight is needed to develop necessary conditions that might help delimit the scope of such a general analysis.

\subsection{Future work}

Work is currently underway to bring the analytical methods of De et al. (2018) to bear on models of much greater complexity than the slot hopper. Specifically, as suggested by Sec. 5.1.1, we favor the application of phaserelated control signals with intention to use averaging to exploit inherent symmetry in Lagrangian systems. We are also exploring applications to a simplified spatial (3dimensional) Minitaur model (lying somewhere between Fig. 1B and C) with the goal of (a) proving its stability in space with these control laws, and (b) proving that the spatial model anchors a slot hopper as suggested in Sec. 5.1.4.

Other current work includes application to a tailed vertical hopper (a constrained version of tail-energized hopping with the Penn Jerboa De and Koditschek (2015a)), as well as a bipedal model with a spine degree-of-freedom such as the one in Culha and Saranli (2011).

\section{Acknowledgements}

The authors would like to thank the anonymous reviewers, whose suggestions greatly improved the paper.

\section{Funding}

This work was supported in part by NSF grant \#1028237, and in part by ONR grant \#N00014-16-1-2817, a Vannevar Bush Fellowship held by the last author, sponsored by the Basic Research Office of the Assistant Secretary of Defense for Research and Engineering.

\section{Supplemental material}

We include a video attachment with trials on Minitaur corresponding to the included data in various plots in this paper, as well as Mathematica scripts containing details of the calculations for analyses.

\section{Notes}

1. For example, for 3D bounding-in-place in Fig. 4, in addition to the two "morphological" parameters per (identical) virtual leg (which only require rough guesses, cf. Fig. 8), the required parameters are $k_{a}$ in (7) (which controls vertical hopping height), and $k_{p}$ and $k_{d}$ in (10) for roll control. If preflexive coordination is insufficient (e.g. bounding-in-place on SCMinitaur in Fig. 13), we compose a phase controller (9) (with one additional parameter). These parameters only require very rough guesses as shown in Sec. 4.1.3.

2. Equivalently, unity eigenvalues have algebraic multiplicity 1 .

3. The reader may note the close correspondence between (Burden et al. 2007, eq. (1)) and the double stance equations of motion we find for the slot hopper (22).

4. We wish to emphasize that our present understanding necessitates in each physical regime a different intuitive choice of the "correct" phase $\psi_{i}$ and the appropriate phase difference $\delta$. Work currently underway is making progress towards replacing this intuitive step with a uniformly defined and algorithmically implemented procedure.

5. Minitaur does not have ab/adduction joints, resulting in its frontal plane projection having rigid leg angles (unlike the vertical legs of the slot hopper in Sec. 2.2). Despite this, pacing Minitaur responds favorably to the phase controller developed on the slot hopper, as shown in Sec. A.6.1.

6. As discussed in Sec. A.6.2, due to the extremely strong coupling between the virtual legs, the retraction policy is needed to separate the phases of the two virtual legs. After this process, each (identical) step is controlled just like a single vertical hopper as analyzed in (De et al. 2018, Sec. 4.1).

7. Note that despite this nomenclature, in some sense the nature of the coupling "changes sign" as the decoupled regime lies at an intermediate $\kappa$-value (Fig. 18).

8. Note that for purposes of analysis, we merely require the coefficient of $u_{1}$ above to be $\mathcal{O}(\varepsilon)$, but for the sake of not introducing a new constant into our perturbation analysis around $\varepsilon=0$, we choose the equality above.

9. Intuitively, in the averaging of a single vertical hopper in De et al. (2018), the introduction of the even function (with respect to the half-orbit center angle $\pi / 2$ ), $\sin \psi_{i}$, in the coordination term, $w_{i}(9)$, leaves a proportionally stabilizing $\delta$ term in $\bar{f}$. However, now with $w_{i}$ set to 0 , the odd function $\left(\cos \psi_{i}\right)$ arising from the vertical energization term in $v_{i}$ (8), can have no effect on relative phase (intentionally, since we prefer the vertical energization control not affect phase relations) and integrates out of the averaged vector field (31). The determination of the "right" phase coordinate $\widetilde{\psi}_{2}(27)$ is, in this light, informed by the computation of $\frac{d}{d t} \widetilde{\delta}$ as to not "disturb" the odd factor $v_{i}$ in (30).

Research is currently under way to facilitate algorithmic generation of slow and fast coordinates suitable for averaging.

10. Lastly, we point out that this decoupling of the vertical and pitch oscillations is in some sense necessary for a limit cycle analysis: as the last two rows of Fig. 9 show, the body angle oscillations generally occur at a different time scale than the stance/flight vertical hopping cycle of $z$, without any sign of phase-locking. 
11. Because larger $\phi$ results in longer unmodeled single stance periods when the active policy of (De et al. 2018, Sec. 2.5) is not applied.

12. Even non-physical $g<0$ values result in observable hopping since the spring-like forces in (7) dominate the constant term in (18).

13. Except the region within the depicted yellow polygon, where the applied radial force (18) is insufficient to overcome gravity and sustain oscillations.

14. Exception is made in the trot (Fig. 10) where the retraction is prescribed for reasons discussed in Sec. A.6.2.

15. For example, into unplanned modes (e.g., double stance Sec. A.6.2) or transitions (e.g. leap from pronk Sec. A.5.3).

16. It is worth noting in passing that there seems to be no analogous path to destabilizing the very strongly preflexive pronking regime (the far right hand side of Fig. 18 with $\kappa$ values well beyond unity) because it is not clear what configuration setpoint to furnish-an observation that might lead us toward consideration of feedforward excited ("clocked") gaits Haynes et al. (2011) or potentially, a refinement via appeal to higher period orbits of the "factored map" expressions.

17. The reported "pronk" observed with the independent state machines in Raibert et al. (1989) is possibly due to the added hip actuation stabilizing the attitude DOFs. Per our analytical result of Sec. 3.2.3, Raibert's quadruped (Raibert et al. 1989, Table 6-1) with only shank actuation would exhibit preflexive bounding stability, as corroborated by the prior simulation study in Murphy and Raibert (1985).

18. A formal morphological reduction in the style of Libby et al. (2015) will be forthcoming when we work on the more formally anchored versions of these controllers in the higher DoF models toward the physical machine.

19. Restricted, in the sense that the prior Berkemeier (1998) analysis-for tractability with conventional methods-had to "ignore" the $\mathcal{O}(\varepsilon)$ dissipation terms which contribute prominently to our vertical hopper (Sec. 2.1.3) and preflexive pronking (Sec. 3.3) stability.

\section{References}

Altendorfer R, Koditschek DE and Holmes P (2004) Stability Analysis of Legged Locomotion Models by SymmetryFactored Return Maps. The International Journal of Robotics Research 23(10-11): 979-999. DOI:10.1177/ 0278364904047389. 27

Barasuol V, Buchli J, Semini C, Frigerio M, De Pieri ER and Caldwell DG (2013) A reactive controller framework for quadrupedal locomotion on challenging terrain. In: Robotics and Automation (ICRA), 2013 IEEE International Conference on. IEEE, pp. 2554-2561. 3
Berkemeier MD (1998) Modeling the dynamics of quadrupedal running. The International Journal of Robotics Research 17(9): 971-985. 2, 3, 4, 7, 27, 28, 29

Biewener A (2003) Animal Locomotion. Animal Locomotion. OUP Oxford. ISBN 978-0-19-850022-3. 2

Brown IE and Loeb GE (2000) A reductionist approach to creating and using neuromusculoskeletal models. Springer, p. 148163. 1

Burden S, Clark J, Weingarten J, Komsuoglu H and Koditschek D (2007) Heterogeneous leg stiffness and roll in dynamic running. In: Proceedings 2007 IEEE International Conference on Robotics and Automation. IEEE, pp. 46454652. 3, 7, 28

Culha U and Saranli U (2011) Quadrupedal bounding with an actuated spinal joint. In: Robotics and Automation (ICRA), 2011 IEEE International Conference on. IEEE, pp. 13921397. 3,28

De A, Burden SA and Koditschek DE (2018) A hybrid dynamical extension of averaging and its application to the analysis of legged gait stability. The International Journal of Robotics Research : 027836491875649DOI:10. 1177/0278364918756498. 1, 2, 4, 5, 6, 9, 10, 11, 12, 13, 17, $20,23,26,27,28,29,35,36$

De A and Koditschek DE (2013) Toward dynamical sensor management for reactive wall-following. In: Robotics and Automation (ICRA), 2013 IEEE International Conference on. IEEE, pp. 2400-2406. 36

De A and Koditschek DE (2015a) Parallel composition of templates for tail-energized planar hopping. In: Robotics and Automation (ICRA), 2015 IEEE International Conference on. pp. 4562-4569. DOI:10.1109/ICRA.2015.7139831. 3, 4, 6, $12,15,27,28$

De A and Koditschek DE (2015b) The Penn Jerboa: A platform for exploring parallel composition of templates. Technical report, University of Pennsylvania. ArXiv:1502.05347. 20

Fukuoka Y and Kimura H (2009) Dynamic locomotion of a biomorphic quadruped Tekken' robot using various gaits: walk, trot, free-gait and bound. Applied Bionics and Biomechanics 6(1): 63-71. DOI:10.1080/11762320902734208. 3

Full RJ and Koditschek DE (1999) Templates and anchors: neuromechanical hypotheses of legged locomotion on land. Journal of Exp. Biology 202(23): 3325-3332. 1, 2, 14, 27

Gan Z, Wiestner T, Weishaupt MA, Waldern NM and Remy CD (2016) Passive dynamics explain quadrupedal walking, trotting, and tlting. Journal of Computational and Nonlinear Dynamics 11(2): 021008. 2

Golubitsky M, Stewart I, Buono PL and Collins JJ (1998) A modular network for legged locomotion. Physica D: Nonlinear Phenomena 115(1): 56-72. 2

Guckenheimer J and Holmes P (1990) Nonlinear Oscillations, Dynamical Systems, and Bifurcations of Vector Fields. Applied Mathematical Sciences. Springer New York. ISBN 
9781461270201.27

Haynes GC, Cohen FR and Koditschek DE (2011) Gait Transitions for Quasi-static Hexapedal Locomotion on Level Ground. In: Pradalier C, Siegwart R and Hirzinger G (eds.) Robotics Research, Springer Tracts in Advanced Robotics, volume 70. Springer Berlin Heidelberg. ISBN 978-3-64219456-6, pp. 105-121. 29

Holmes P (2005) Ninety plus thirty years of nonlinear dynamics: Less is more and more is different. International Journal of Bifurcation and Chaos 15(09): 2703-2716. 27

Hutter M, Sommer H, Gehring C, Hoepflinger M, Bloesch M and Siegwart R (2014) Quadrupedal locomotion using hierarchical operational space control. The International Journal of Robotics Research 33(8): 1047-1062. DOI:10. 1177/0278364913519834. 3

Johnson AM, Burden SA and Koditschek DE (2016) A hybrid systems model for simple manipulation and self-manipulation systems. The International Journal of Robotics Research 35(11): 1354-1392. DOI:10.1177/0278364916639380. 7, 8, 16

Kenneally G, De A and Koditschek DE (2016) Design Principles for a Family of Direct-Drive Legged Robots. IEEE Robotics and Automation Letters 1(2): 900-907. DOI:10.1109/LRA. 2016.2528294. 2, 14, 20, 21, 34

Kenneally G and Koditschek DE (2017) Characterization of Robotic Transparency and Energetic Cost of Proprioceptive Information. In: in prep. 21

Klavins E, Koditschek DE and Ghrist R (2000) Toward the Regulation and Composition of Cyclic Behaviors. In: Donald B, Lynch K and Rus D (eds.) Algorithmic and Computational Robotics: New Directions 2000 WAFR. Wellesley: A.K. Peters, pp. 205-220. 2, 5

Klavins E, Komsuoglu H, Full RJ and Koditschek DE (2002) The Role of Reflexes versus Central Pattern Generators in Dynamical Legged Locomotion. In: Neurotechnology for Biomimetic Robots. MIT Press, Cambridge, MA, pp. 351382. 2, 3

Koditschek DE (1987) Adaptive techniques for mechanical systems. In: Proc. 5th. Yale Workshop on Adaptive Systems. pp. 259-265. 14

Koditschek DE and Buehler M (1991) Analysis of a simplified hopping robot. The International Journal of Robotics Research 10(6): 587-605. DOI:10.1177/027836499101000601. 5,13

Libby T, Johnson AM, Chang-Siu E, Full RJ and Koditschek DE (2015) Comparative design, scaling, and control of appendages for inertial reorientation. ArXiv preprint arXiv:1511.05958 [cs.RO]. 7, 29

Lopes GAD and Koditschek DE (2007) Visual Servoing for Nonholonomically Constrained Three Degree of Freedom Kinematic Systems. The International Journal of Robotics Research 26(7): 715-736. DOI:10.1177/0278364907080737.
36

Minitaur (2016) Ghost robotics minitaur. Http://www.ghostrobotics.io/minitaur/. 2, 4, 14

Murphy KN and Raibert MH (1985) Trotting and bounding in a planar two-legged model. In: Theory and Practice of Robots and Manipulators. Springer, pp. 411-420. 2, 7, 21, 25, 27 , 28,29

Neunert M, Farshidian F, Winkler AW and Buchli J (2016) Trajectory Optimization Through Contacts and Automatic Gait Discovery for Quadrupeds. arXiv preprint arXiv:1607.04537 . 3

Park HW, Wensing PM and Kim S (2017) High-speed bounding with the mit cheetah 2: Control design and experiments. The International Journal of Robotics Research 36(2): 167-192. 3,6

Poulakakis I (2005) Modeling and Experiments of Untethered Quadrupedal Running with a Bounding Gait: The Scout II Robot. The International Journal of Robotics Research 24(4): 239-256. DOI:10.1177/0278364904050917. 2

Poulakakis I (2006) On the Stability of the Passive Dynamics of Quadrupedal Running with a Bounding Gait. The International Journal of Robotics Research 25(7): 669-687. DOI:10.1177/0278364906066768. 2

Pusey JL, Duperret JM, Haynes GC, Knopf R and Koditschek DE (2013) Free-standing leaping experiments with a powerautonomous elastic-spined quadruped. In: SPIE Defense, Security, and Sensing. International Society for Optics and Photonics, pp. 87410W-87410W. 3

Raibert M (1986) Legged Robots that Balance. Artificial Intelligence. MIT Press. ISBN 9780262181174. 2, 13, 21, 24, 25, 27, 28, 32, 33, 36

Raibert MH, Brown Jr HB, Chepponis M, Koechling J, Hodgins JK, Dustman D, Brennan WK, Barrett DS, Thompson CM, Hebert JD and others (1989) Dynamically Stable Legged Locomotion (September 1985-Septembers1989) . 2, 3, 20, $21,26,27,29,32,33,35,36$

Righetti L and Ijspeert AJ (2008) Pattern generators with sensory feedback for the control of quadruped locomotion. In: Robotics and Automation, 2008. ICRA 2008. IEEE International Conference on. pp. 819-824. 3

Saranli U and Koditschek DE (2003) Template based control of hexapedal running. In: Robotics and Automation, 2003. Proceedings. ICRA'03. IEEE International Conference on, volume 1. IEEE, pp. 1374-1379. 13, 21, 36

Schmitt J and Holmes P (2000) Mechanical models for insect locomotion: dynamics and stability in the horizontal plane $i$. theory. Biological cybernetics 83(6): 501-515. 27

Secer G and Saranli U (2013) Control of monopedal running through tunable damping. In: 2013 21st Signal Processing and Communications Applications Conference (SIU). pp. 14. DOI:10.1109/SIU.2013.6531557. 6 
Shahbazi M and Lopes GAD (2016) Coordination of Monopedal SLIP Models Towards Quadrupedal Running. In: 2016 IEEE/RSJ International Conference on Intelligent Robots and Systems (IROS). Daejeon, Korea. 2

Shkolnik A, Levashov M, Manchester IR and Tedrake R (2011) Bounding on rough terrain with the LittleDog robot. The International Journal of Robotics Research 30(2): 192-215. DOI:10.1177/0278364910388315. 3

Sprowitz A, Tuleu A, Vespignani M, Ajallooeian M, Badri E and Ijspeert AJ (2013) Towards dynamic trot gait locomotion: Design, control, and experiments with Cheetahcub, a compliant quadruped robot. The International Journal of Robotics Research 32(8): 932-950. DOI:10.1177/ 0278364913489205. 3, 34

Sutherland IE and Carlson FH (1983) A walking robot. Sutherland, Sproull \& Ass. 2

Symon K (1971) Mechanics. Addison-Wesley World student series. Addison-Wesley Publishing Company. ISBN 978-0201-07392-8. 8

Weingarten JD, Groff RE and Koditschek DE (2004) A framework for the coordination of legged robot gaits. In: Robotics, Automation and Mechatronics, 2004 IEEE Conference on, volume 2. pp. 679-686. 34

\section{A Appendix}

This appendix contains various calculations that are used for our stability proofs, broken down by the section in which they appear.

\section{A.1 WC averaged return map eigenvalues}

Now we calculate the eigenvalues of (35). The characteristic polynomial is

$$
\begin{aligned}
m_{\varepsilon}(\lambda) & =\lambda^{3}-\lambda^{2}\left(-1+\varepsilon \zeta_{4}\right)-\zeta_{5}\left(1-\varepsilon \zeta_{4}\right) \lambda-\zeta_{5} \\
& =: \lambda^{3}+c_{2}(\varepsilon) \lambda^{2}+c_{1}(\varepsilon) \lambda+c_{0}(\varepsilon) .
\end{aligned}
$$

The roots are an $\varepsilon$-parameterized family of scalars $\rho$ : $\mathbb{R}_{>0} \rightarrow \mathbb{C}$. The eigenvalues at $\varepsilon=0$ are the roots of $m_{0}(\lambda)=\lambda^{3}+\lambda^{2}-\lambda-1=\left(\lambda^{2}-1\right)(\lambda+1)$ (noting that $\left.\zeta_{5}\right|_{\varepsilon=0}=1$ from (36)), which are $\{-1,-1,1\}$, and thus $\rho_{1}(0)=-1, \rho_{2}(0)=1, \rho_{3}(0)=-1$.

Now we consider two cases: as $\varepsilon>0$, either the eigenvalues remain all real, or two of them move into the complex plane as a complex conjugate pair:

i) $\rho_{i} \in \mathbb{R}$ : To do this, we find $\rho_{i}^{\prime}(0)$. Note that the characteristic polynomial is alternatively expressed as

$$
\begin{array}{r}
m_{\varepsilon}(\lambda)=\Pi_{i=1}^{3}\left(\lambda-\rho_{i}(\varepsilon)\right)=\lambda^{3}+\left(-\rho_{1}-\rho_{2}-\rho_{3}\right) \lambda+ \\
\left(\rho_{1} \rho_{2}+\rho_{1} \rho_{3}+\rho_{2} \rho_{3}\right) \lambda-\rho_{1} \rho_{2} \rho_{3} .
\end{array}
$$

Using the chain rule, we equate $\mathrm{D}_{\varepsilon} c(\varepsilon)=\mathrm{D}_{\rho} c(\varepsilon)$. $\rho^{\prime}(\varepsilon)$ and evaluate at $\varepsilon=0$. However, the matrix $\left.\mathrm{D}_{\rho} c(\varepsilon)\right|_{\varepsilon=0}$ is singular, indicating that not all the eigenvalues remain real as $\varepsilon>0$.

ii) $\rho_{1} \in \mathbb{R}, \rho_{2}, \rho_{3} \in \mathbb{C}:$ We reparameterize $\rho_{2}, \rho_{3}=$ $\zeta_{6} \pm j \zeta_{7}$, and define $\zeta_{8}:=\zeta_{6}^{2}+\zeta_{7}^{2}$. The characteristic polynomial is now

$$
\begin{aligned}
m_{\varepsilon}(\lambda)=\Pi_{i=1}^{3}\left(\lambda-\rho_{i}(\varepsilon)\right)= & \lambda^{3}+\left(-\rho_{1}-2 \zeta_{6}\right) \lambda+ \\
& \left(2 \rho_{1} \zeta_{6}+\zeta_{8}\right) \lambda-\rho_{1} \zeta_{8} .
\end{aligned}
$$

We can equate the coefficients with (56), and differentiate with $\varepsilon$ on both sides to get

$$
\begin{aligned}
&-2 \zeta_{6}^{\prime}-\rho_{1}^{\prime}=c_{2}^{\prime}, \\
& \zeta_{8}^{\prime}-2 \zeta_{6} \rho_{1}^{\prime}+2 \zeta_{6}^{\prime} \rho_{1}=c_{1}^{\prime}, \\
&-\rho_{1}^{\prime} \zeta_{8}-\rho_{1} \zeta_{8}^{\prime}=c_{0}^{\prime},
\end{aligned}
$$

where' denotes $\varepsilon$-derivative. Now evaluating both sides at $\varepsilon=0$, we get a linear system of equations

$$
\left[\begin{array}{ccc}
-1 & 0 & -2 \\
-2 & 1 & 2 \\
-1 & -1 & 0
\end{array}\right]\left[\begin{array}{l}
\rho_{1}^{\prime}(0) \\
\zeta_{8}^{\prime}(0) \\
\zeta_{6}^{\prime}(0)
\end{array}\right]=c^{\prime}(0),
$$

which is readily solved to yield $\rho_{1}^{\prime}(0)=-\frac{\beta}{4 \omega}$, and $\zeta_{8}^{\prime}(0)=-\frac{\beta}{4 \omega}$. Thus the first-order Taylor expansion of the roots is

$$
\begin{aligned}
\rho_{1} & =1-\frac{\varepsilon \beta}{4 \omega}+\mathcal{O}\left(\varepsilon^{2}\right), \\
\left|\rho_{2}\right|^{2}=\left|\rho_{3}\right|^{2} & =1-\frac{\varepsilon \beta}{4 \omega}+\mathcal{O}\left(\varepsilon^{2}\right),
\end{aligned}
$$

and we are guaranteed that all the roots move inside the unit circle for small $\varepsilon>0$.

\section{A.2 SC decoupling under Assumption 2}

Substituting our controllers (7), (8) with the attitude controller $(10)^{20}$ into the double stance equations of motion (22), we see that in closed loop,

$$
\begin{gathered}
\ddot{z}=\omega^{2}(\rho-z)-\varepsilon \beta \dot{z}-\varepsilon k_{a}\left(\cos \psi_{1}+\cos \psi_{2}\right) / 2, \\
\kappa \ddot{\phi}=-d\left(\omega^{2}+k_{p}\right) \phi-d\left(\varepsilon \beta+k_{d}\right) \dot{\phi} \\
-\varepsilon k_{a}\left(\cos \psi_{1}-\cos \psi_{2}\right) / 2 .
\end{gathered}
$$

Now we simplify the sum and difference cosines in the equation above. Define the variables $p_{i}:=p\left(z_{i}, \dot{z}_{i}\right)$ (where the map $p$ is as defined in Sec. 2.1.2), and $p_{z}:=p(z, \dot{z})$. From (24), note that $p_{1}=p_{z}+p_{\phi}$, and $p_{2}=p_{z}-p_{\phi}$, where $p_{\phi}$ is defined in Sec. 3.3. To approximate $\cos \psi_{i}$ in (57), we need to approximate $1 /\left\|p_{i}\right\|$ :

$$
\begin{aligned}
\frac{1}{\left\|p_{z}+p_{\phi}\right\|^{2}} & =\frac{1}{p_{z}^{T} p_{z}+p_{\phi}^{T} p_{\phi}+2 p_{z}^{T} p_{\phi}} \\
& =\frac{1}{\left\|p_{z}\right\|^{2}}\left(\frac{1}{1+\left(a_{\phi} / a\right)^{2}+p_{z}^{T} p_{\phi} / a^{2}}\right)
\end{aligned}
$$


where note that from assumption 2, $\left(a_{\phi} / a\right)^{2}+$ $p_{z}^{T} p_{\phi} / a^{2}=\mathcal{O}(\varepsilon)$. Taking a square root, we get

$$
\frac{1}{\left\|p_{z}+p_{\phi}\right\|}=\frac{1}{\left\|p_{z}\right\|}\left(1-\delta_{+} / 2\right)+\mathcal{O}(\varepsilon)
$$

where we define $\delta_{+}:=\left(a_{\phi} / a\right)^{2}+p_{z}^{T} p_{\phi} / a^{2}$. Similarly, we can calculate

$$
\frac{1}{\left\|p_{z}-p_{\phi}\right\|}:=\frac{1}{\left\|p_{z}\right\|}\left(1-\delta_{-} / 2\right)+\mathcal{O}(\varepsilon),
$$

where $\delta_{-}:=\left(a_{\phi} / a\right)^{2}-p_{z}^{T} p_{\phi} / a^{2}$. We can now use these to calculate

$$
\begin{aligned}
& \cos \psi_{1}+\cos \psi_{2}=e_{1}^{T}\left(\frac{p_{1}}{\left\|p_{1}\right\|}+\frac{p_{2}}{\left\|p_{2}\right\|}\right) \\
& =\frac{e_{1}^{T}}{\left\|p_{z}\right\|}\left(\left(p_{z}+p_{\phi}\right)\left(1-\delta_{+} / 2\right)+\left(p_{z}-p_{\phi}\right)\left(1-\delta_{-} / 2\right)\right) \\
& =\frac{e_{1}^{T}}{\left\|p_{z}\right\|}\left(p_{z}\left(2-\frac{\delta_{+}+\delta_{-}}{2}\right)-p_{\phi} \frac{\delta_{+}-\delta_{-}}{2}\right)+\mathcal{O}(\varepsilon) \\
& =\cos \psi\left(2+\mathcal{O}\left(\varepsilon^{2}\right)\right)+\mathcal{O}(\varepsilon)=2 \cos \psi+\mathcal{O}(\varepsilon)
\end{aligned}
$$

since $a_{\phi} / a$ appears in the $p_{\phi}$ term (use Assumption 2 again). Similarly,

$$
\begin{aligned}
& \cos \psi_{1}-\cos \psi_{2} \\
& =\frac{e_{1}^{T}}{\left\|p_{z}\right\|}\left(p_{\phi}\left(2-\frac{\delta_{+}+\delta_{-}}{2}\right)-p_{z} \frac{\delta_{+}-\delta_{-}}{2}\right)+\mathcal{O}(\varepsilon) \\
& =\mathcal{O}(\varepsilon)-\cos \psi\left(p_{z}^{T} p_{\phi} / a^{2}\right)=\mathcal{O}(\varepsilon),
\end{aligned}
$$

using Assumption 2 again. Using (58) and (59) in (57),

$$
\begin{aligned}
\ddot{z} & =\omega^{2}(\rho-z)-\varepsilon \beta \dot{z}-\varepsilon k_{a} \cos \psi_{z}, \\
\kappa \ddot{\phi} & =-d\left(\omega^{2}+k_{p}\right) \phi-d\left(\varepsilon \beta+k_{d}\right) \dot{\phi},
\end{aligned}
$$

where $\psi_{z}=\angle p(z, \dot{z})$.

\section{A.3 SC preflexive attitude stability: $P_{\phi}$ is a contraction}

We next prove that $P_{\phi}$ is a contraction subject to Assumption 3. First, we need a Lemma:

Lemma 2. For $\chi \geq 0, e^{\chi} \geq 1+\chi^{2} / 2+\chi \sqrt{1+(\chi / 2)^{2}}$.

Proof. First, note that

$$
\begin{array}{r}
\frac{d}{d \chi}\left(1+\frac{\chi^{2}}{2}+\chi \sqrt{1+\chi^{2} / 4}\right)=\chi+\frac{2+\chi^{2}}{\sqrt{4+\chi^{2}}} \\
\leq \chi+\frac{2+\chi^{2}}{2}=1+\chi+\frac{\chi^{2}}{2} \leq e^{\chi}
\end{array}
$$

whereas the last expression is the derivative of $e^{\chi}$. Since both expressions equal 1 at $\chi=0$, and the derivative of $e^{\chi}$ is pointwise greater for $\chi \geq 0, e^{\chi}$ is the larger expression for all $\chi \geq 0$.

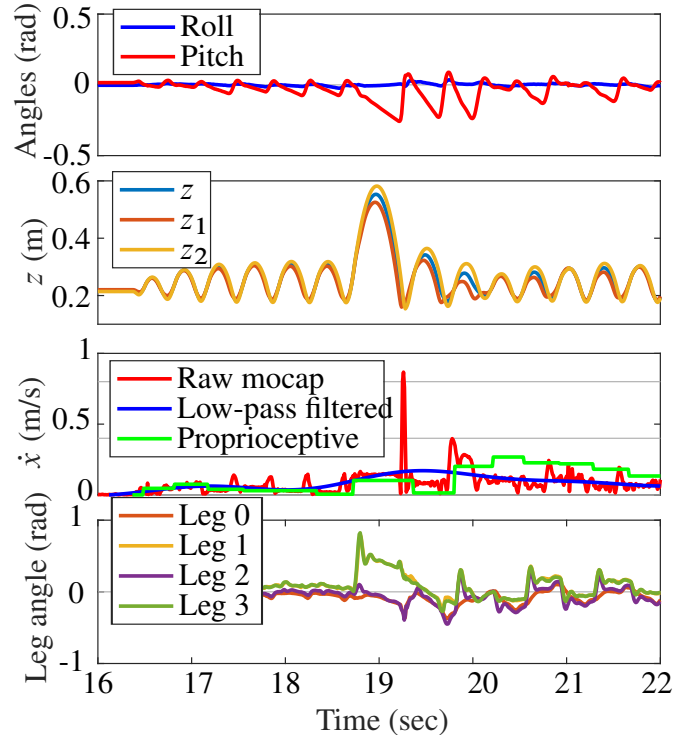

Figure 19. Minitaur pronking leap. Empirical data from Minitaur (Fig. 1C) exhibiting pronking (10), with a discrete "leap" command send at $t=18.5$ seconds (higher desired vertical energy for a single stride at both hips) resulting in a vertical displacement of the center of mass while pronking.

Let us define $\theta_{\mathrm{s}}:=\omega_{\phi} t_{\mathrm{s}}$, and $\theta_{\mathrm{f}}:=\omega_{\phi} t_{\mathrm{f}}$. To check if $P_{\phi}$ is a contraction, we need to check that the largest eigenvalue of $P_{\phi}^{T} P_{\phi}$ lies within the unit cycle, since this is the largest value that $y^{T} P_{\phi}^{T} P_{\phi} y / y^{T} y$ can take. The largest eigenvalue is

$$
\lambda_{\max } \leq \frac{e^{-2 \beta_{\phi} \theta_{\mathrm{s}}}}{2}\left(2+\theta_{\mathrm{f}}^{2}+\theta_{\mathrm{f}} \sqrt{4+\theta_{\mathrm{f}}^{2}}\right) \leq e^{-2 \beta_{\phi} \theta_{\mathrm{s}}+\theta_{\mathrm{f}}},
$$

where we use the Lemma above for the last inequality. Since the exponential is monotonic, we only need check

$$
-2 \beta_{\phi} \theta_{\mathrm{s}}+\theta_{\mathrm{f}}<0 \Longleftrightarrow t_{\mathrm{f}}-\frac{\varepsilon \pi \beta \sqrt{d / \kappa}}{\omega^{2}}<0
$$

which is ensured by Assumption 2.

\section{A.4 Virtual leg groups: our implementation compared to that of Raibert et al. (1989)}

a) Event synchronization.

Our implementation. As shown in Fig. 10, each virtual leg has an independent mode, STANCE or FLIGHT. Transitions between these modes are triggered by events: TOUCHDOWN (described in Sec. 4.2.2) and LIFTOFF (elapse of $\pi / \omega$ seconds since TOUCHDOWN, following the constant flowtime assumption (Table 2). The specific STANCE and FLIGHT controllers are presented next.

Comparison to Raibert. This part of our implementation is a simpler version of the "Synchronization" rule in (Raibert 1986, pg. 93). Our justification for 
this is our comparative numerical work in Sec. 4.1.2 showing that the qualitative effect of active toe extension is relatively minor. Additionally, feedback synchronization in flight relies heavily on the ground being particularly flat.

b) Mean-difference coordinates in stance.

Our implementation. Our analytical result of Sec. 3.3.7 showed that using only shank extension actuators, (10) can be applied to two stance legs to feedback-stabilize the body orientation for the inphase (pronking template) limit cycle. Noting from (10) that the two legs receive equal and opposite control signals, we introduce the following "meandifference" input coordinate change:

For a desired virtual leg radial force $\Upsilon_{1}$ (given by (18)), suppose the physical radial forces commanded of the within-group legs are $\Upsilon_{1+}$ and $\Upsilon_{1-}$ in a manner we now specify. In Fig. 11, we show which legs are grouped together into virtual legs for each of the gaits discussed in this paper, as well as the leg numbering scheme we use for Minitaur. E.g., in the bounding gait, legs " 0 " and " 2 " receive radial actuation signals $\Upsilon_{1+}$ and $\Upsilon_{1-}$, and legs " 1 " and " 3 " receive radial actuation signals $\Upsilon_{2+}$ and $\Upsilon_{2-}$, respectively.

Additionally, define the attitude controller for withingroup stabilization

$$
\Upsilon_{\mathrm{wg}}:=k_{p} \phi_{\mathrm{wg}}+k_{d} \dot{\phi}_{\mathrm{wg}},
$$

where $\phi_{\mathrm{wg}}$ is the body angle in the plane containing the within-group legs (e.g. for bounding, $\phi_{\mathrm{wg}}$ is the roll angle; for pacing, $\phi_{\mathrm{wg}}$ is the pitch angle; for trotting, $\phi_{\mathrm{wg}}$ is the body angle projected in the plane containing the diagonally paired legs). Then, in stance the physical legs are commanded the radial forces

$$
\Upsilon_{1+}:=\Upsilon_{1}+\Upsilon_{\mathrm{wg}}, \quad \Upsilon_{1-}:=\Upsilon_{1}-\Upsilon_{\mathrm{wg}} .
$$

We point out here the $\phi_{\mathrm{wg}}$ is not visible in right column of Fig. 11 since it is the angle of the body about an axis on the plane of the page. We also observe that (61) is a mere copy of our previously defined attitude controller (10) (after Assumption 1 is incorporated), simply applied about a different axis. We define it separately here in order to be explicit about within-group feedback stabilization in the remainder of the paper.

Comparison to Raibert. This part of our implementation differs from the "Force Equalization" rule in (Raibert 1986,pg. 93), since we explicitly command radial forces that are unequal across the leg groups and utilize this affordance for feedback attitude stabilization. A later account of Raibert's implementation

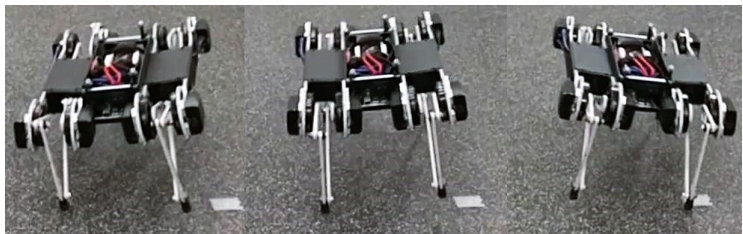

Figure 20. Snapshots of pacing in place (taken roughly at left stance, aerial, right stance) from the experiment in Fig. 23(center, right).

on his quadruped Raibert et al. (1989) reveals that two additional actuators (which we don't need with our method) are used for feedback attitude stabilization (see Sec. A.6.2 for further details about trot implementation).

c) Toe positioning in flight.

Our implementation. In flight, the radial forces simply act to keep each toe at the nominal leg extension, $\rho$. The hip actuators are used to servo the toes to a desired absolute leg angle, $\theta^{*}$ using the controller described in (63). We furnish additional details about the hip actuation in Sec. A.5.1.

Comparison to Raibert. This part of our implementation is equivalent to the "Positioning" rule in (Raibert 1986, pg. 92).

\section{A.5 Empirical compositions}

A.5.1 Fore-aft speed control We adapt the monopedal fore-aft speed controller described in Raibert (1986), and we review it briefly here. Our decoupled control strategy (7) already encourages thinking of sagittal plane Minitaur as a composition of a "front" and "rear" monoped, and so we apply the fore-aft control to each monoped independently. The control input is the touchdown angle of the virtual leg in flight, which is easy to actuate with very little energetic cost by using the hip actuators, since the toes are light. Specifically, we set

$$
\ddot{\theta}_{i}=k_{p}\left(\theta^{*}(\dot{x})-\theta_{i}\right)-k_{d} \dot{\theta}_{i}
$$

where the desired touchdown angle, $\theta^{*}(\dot{x})$ is defined as a function of the current speed $\dot{x}$ according to the "neutral point" ideas of Raibert (1986),

$$
\rho \sin \left(\theta^{*}(\dot{x})\right)=-\frac{\pi \dot{x}}{2 \omega}+k_{\dot{x}}\left(\dot{x}^{*}-\dot{x}\right)
$$

for $i \in \mathcal{J}$, where note that $\pi / \omega$ is the stance duration (assumed constant as in Table 2). Recall that $\theta_{i}$ is the absolute leg angle, and an estimate of the body pitch, $\phi$, is required as well to command the leg angle correctly.

An important part of this controller is an estimate of the current speed, $\dot{x}$. We obtain a proprioceptive forward speed estimate in Minitaur by using encoder-measured joint 


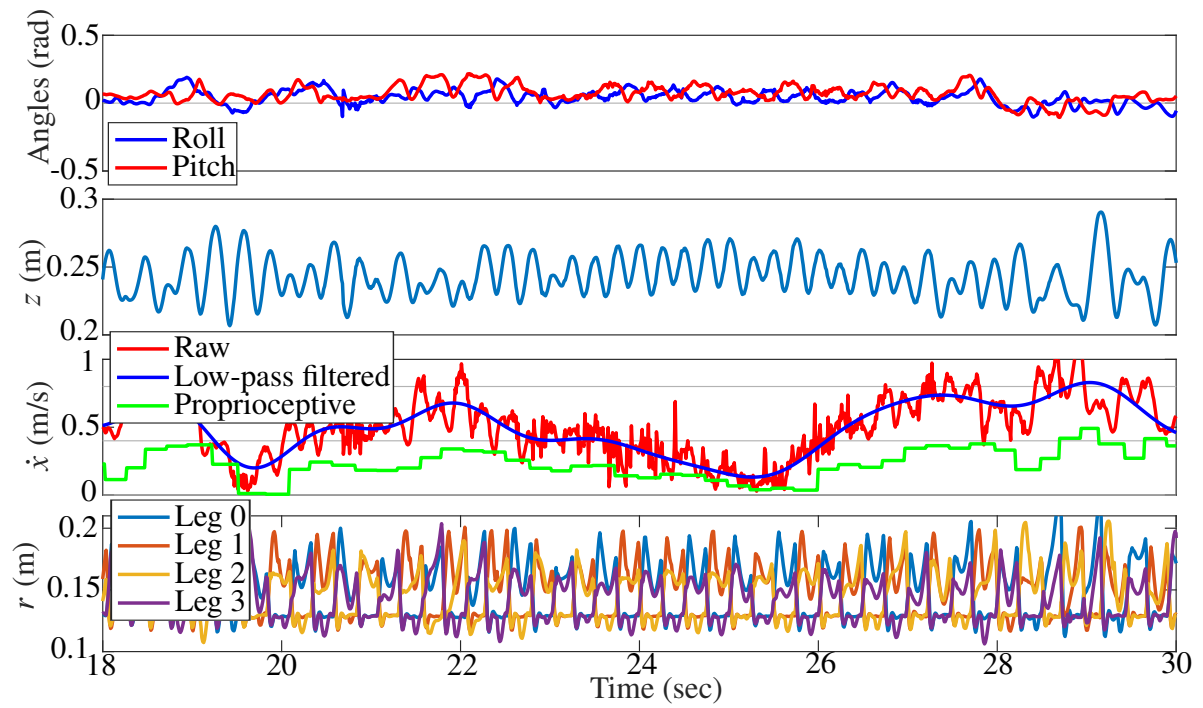

Figure 21. Minitaur feedback-stabilized trotting. Empirical data from WC-Minitaur exhibiting trotting implemented by commanding two diagonally paired virtual legs as vertical hoppers (7), but with added coordination logic (Sec. A.6.2).

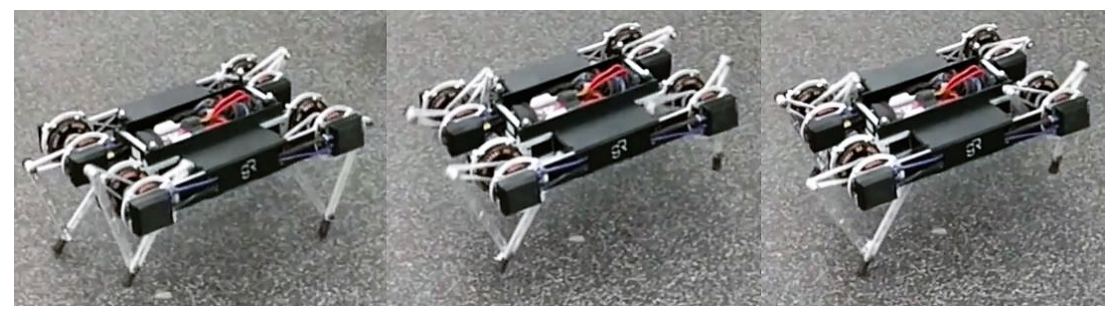

Figure 22. Snapshots of trotting showing legs 1 and 2 (numbering shown in Fig. 11) in stance (left), aerial (center), and then legs 0 and 3 in stance (right). Going from the left panel to the center panel, the active retraction of legs 1 and 2 (as described in Sec. A.6.2) is also apparent. These snapshots are from the experiment in Fig. 21.

angles and velocities together with the forward kinematics $g: \mathbb{R}_{+} \times S^{1} \rightarrow \mathbb{R}^{2}$ of the leg,

$$
\dot{x}=\mathrm{D} g\left(r_{i}, \theta_{i}\right)\left[\begin{array}{c}
\dot{r}_{i} \\
\dot{\theta}_{i}
\end{array}\right]
$$

for $i \in \mathcal{J}$, where $\mathrm{D} g$ is the leg Jacobian derived in (Kenneally et al. 2016, eqn. (6)).

Applying this controller to preflexively bounding Minitaur (shown bounding in place in Fig. 4) along with a roll controller (61) to stabilize out-of-sagittal-plane motion, results in fore-aft motion as shown in Fig. 15, while not disturbing the front/rear preflexive phase coordination at a range of speeds. The desired speed $\dot{x}^{*}$ is set by an external signal (often an operator, but in this case we used a ramp function showed in dashed black). In the last row of Fig. 15, we use a $3 \mathrm{~Hz}$ cutoff filter to low-pass the raw speed measurements from motion capture. The step rate of Minitaur (each front or rear stance is counted as a "step") is about $3 \mathrm{~Hz}$, and a distinct oscillation can be noticed at around this frequency in the forward speed. We hypothesize that this is an artifact of imperfect tuning between the front and rear hips; a full analysis of the fore-aft stability (that we defer to future work) should illuminate this issue further.

Even with this decoupled compositional control, it is still possible to attain traveling speeds of $1.92 \mathrm{~m} / \mathrm{s}(4.8$ body lengths / second, or 11 leg lengths / second), comparing favorably to quadrupeds of a similar size (as listed in Sprowitz et al. (2013)), keeping in mind that Minitaur is a general-purpose quadruped with onboard power. Further, since this gait is the result of a smoothly parametrized family of control policies (7), (61) (rather than, e.g., empirically optimized parameter set points as in Weingarten et al. (2004)), stable bounding can be accessed at a continuum of traveling speeds and yaw rates (as demonstrated in Fig. 15), as well as vertical height (shown below in Sec. A.5.3). We posit that the limit to even higher speeds in these trials is primarily due to:

a) inaccuracy in $\dot{x}$ estimate: as observed in Fig. 15, the proprioceptive speed estimate (64) systematically underestimates the actual speed of the robot past around $1.5 \mathrm{~m} / \mathrm{s}$. This usually results in incorrect toe placement, and the robot stumbles forward. The 


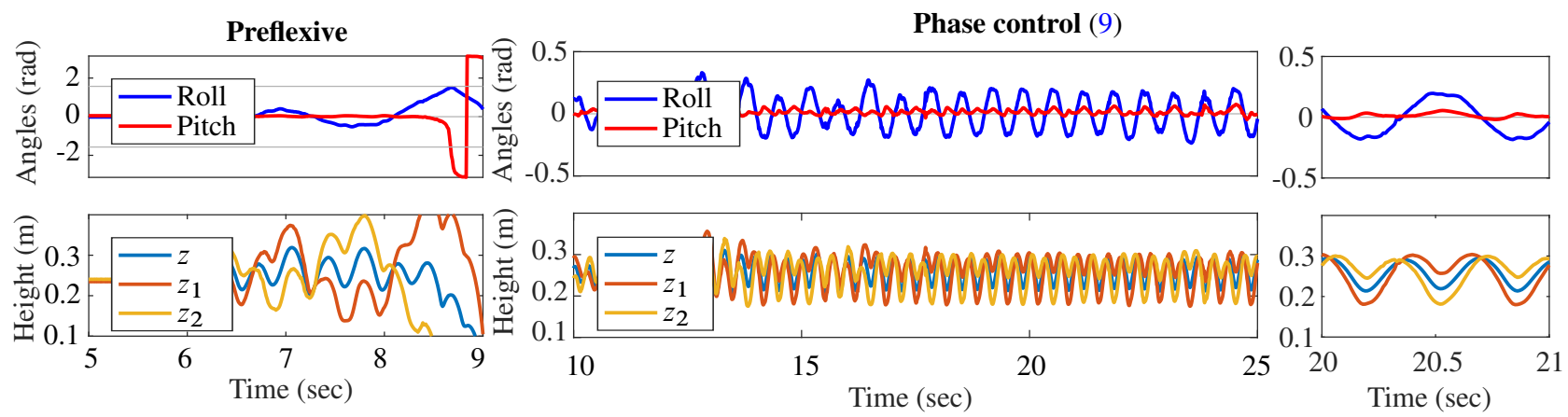

Figure 23. Minitaur preflexive and feedback-stabilized pacing. Empirical data from Minitaur (Fig. 1C) with decoupled vertical hopping controllers applied to "left" and "right" virtual hips, revealing no preflexive stability (left) resulting in a roll over (note faint horizontal lines are at $\pm \pi / 2$, and the IMU Euler angles pass through a parameterization singularity), but a stable roll oscillation (center, right) with our feedback phase controller (9). The zoomed-in segment (right) reveals interesting comparisons to preflexive (Fig. 4) and forced (Fig. 13) sagittal plane bounding. See Sec. A.6.1 for discussion.

reason for this inaccuracy could be toe slip (see next point) or minor delays in estimation of touchdown and liftoff (which are on the order of 5-10 ms as stated in Sec. 4.2);

b) loss of traction: yaw correction in stance (Sec. A.5.2) requires a large amount of friction, and especially at higher speeds small amounts of toe slip can cause failure;

c) destabilization of pitch oscillation: the preflexive stabilization we analyzed in Sec. 3.2 maintains the desired pitch oscillations for a range of speeds, but as seen between $t \in[52,56]$ seconds in Fig. 15, the pitch oscillations can reduce in magnitude, or worse, get out of synchronization with the leg touchdowns when a lot of energy is injected at higher traveling speeds.

The last point motivates us to incorporate the fore-aft degree of freedom in our analysis in the future, as well as to augment the bounding coordination with additional coordination control.

While the phase controller (9) is successful at disrupting preflexive pronking in SC-Minitaur (Sec. 13), it is unable to disrupt preflexive bounding (as we observed in Sec. 4.4). Likewise, we did not observe any empirical benefit to the application of (9) to "augment" Minitaur's preflexive bounding stability (as analyzed in Sec. 3.2). However, we posit that for a physical machine with $\kappa \approx 1$ (no preflexive stability), (9) could be used to feedback-stabilize bounding (as analyzed in De et al. (2018)).

The fore-aft control above is also applied to pronking (Fig. 14) as well as trotting (below, in Sec. A.6.2) to obtain modest traveling speeds of 1 and 2 body lengths / second respectively. The inaccuracy in the proprioceptive speed estimate was much larger in these other gaits, so we believe that these gaits can travel at much faster speeds with some more tuning. Since the focus of this paper is the coordination control of the $z, \phi$ degrees of freedom, we defer to future work the analysis of vertical and fore-aft coupling that would facilitate extracting faster translational speeds from these other gaits.

A.5.2 Yaw control while bounding We also compose an empirically motivated yaw controller, that makes use of the available hip torques in stance. Specifically, the two legs comprising the "virtual leg" in stance apply differential hip torques in order to impart a yawing moment on the body (in the horizontal plane).

If $\zeta \in S^{1}$ is the yaw angle, $\dot{\zeta}^{*} \in \mathbb{R}$ is the desired yaw rate, and $\tau_{j}$ is the hip torque applied to the left and right hips in stance, we set

$$
\tau_{j}=(-1)^{j} k_{\dot{\zeta}} \dot{\zeta}^{*}
$$

where $j \in\{$ left, right $\}$. Note that we intentionally use a different index than $i \in \mathcal{J}$ used in Sec. 2.1.2 and throughout the paper to refer to the two virtual legs, since in (65) we are referring to legs grouped within an erstwhile "single" virtual leg.

This is a different strategy than the horizontal-plane toe placement strategy in Raibert et al. (1989), which requires 2DOF control of toe position in the horizontal plane (an ab/adduction joint) and is thus inaccessible to Minitaur.

This strategy results in controllable yaw in bounding Minitaur. As an example, in Fig. 15, the yaw of the robot begins to drift at around $t=54$ seconds, and the yaw controller (with desired yaw being supplied by an operator) allows the robot to the brought back on course. As expected, this introduces a rolling disturbance that can be seen at around $t=56$ seconds, but the roll is eventually stabilized by the attitude controller (61).

Further characterization of the fore-aft and yaw controllers is needed to argue that bounding Minitaur 
anchors a unicycle in the horizontal plane; we defer this topic to future work. Experiments in the lab are already underway employing higher-level control schemes designed for unicycle plants, such as Lopes and Koditschek (2007); De and Koditschek (2013).

A.5.3 Running leaps As mentioned in Sec. A.5.1, our template-based control (7) affords the operator the ability to control the height setpoint $a^{*}$ through $k_{a}$. Even though in our analysis we have kept $a^{*}$ constant, in practice, we can increase $a^{*}$ for a single stride to get a running leap. This results in a large perturbation off the limit cycle (both in bounding and pronking), but the large stability basin of the (preflexive or feedback) coordination of Sec. 4.3-4.4 helps restore the nominal gait pattern within 1-2 strides.

Fig. 16 shows a bound leap: though there is a large pitching disturbance before the body recovers, the fore-aft speed of the body as well as the $z_{1}, z_{2}$ stepping pattern is maintained through the leap, demonstrating the large stability of basins of the template controllers. Fig. 19 shows a pronk leap at around $t=18.5$ seconds, where again a large pitching disturbance is introduced by the leap, but the $z$-oscillations are not disturbed.

\section{A.6 Pacing and trotting details}

A.6.1 Pacing We find that in the frontal plane, when the decoupled controller (7) is applied to the left and right virtual legs, Minitaur does not preflexively exhibit any coordination. This leads us to believe that it lies in the "chaotic" region on Fig. 18. The left column of Fig. 23 reveals typical preflexive behavior: a fall within a few steps.

However, when our phase controller (9) is applied (suggested by our analytical insight from De et al. (2018)), a stable anti-phase limit cycle emerges in the frontal plane. We reiterate here that since Minitaur does not have an ab/adduction joint, the virtual hips canot be moved by using hip torques in the frontal plane. Thus, this stabilization is established only using the leg extension actuators, using the phase control ideas we have described in Sec. 2.3.

A.6.2 Trotting In a trot, the virtual hips are located close to the center of mass Raibert (1986), effectively making $d$ very small. Consequently, from $(13), \kappa$ is very large, and as Fig. 18 shows, the in-phase limit cycle is preflexively stable.

This preflexive stability is very strong, and phase control (9) as in Sec. 4.4.1 is unable to produce a trot. The trot implementation in Raibert et al. (1989) used a sequenced state machine, logically forcing alternating virtual leg stances. We have employed a slightly less aggressive strategy (Fig. 10), where the virtual leg state machine is now updated to include 3 possible states: STANCE, FLIGHT, and RETRACT. For $i \in \mathcal{J}$, when virtual leg $i$ goes through its liftoff event, it is placed in the RETRACT state where it is kinematically prevented from touching down. When leg $i+1$ has lifted off, leg $i$ is moved from RETRACT $\rightarrow$ FLIGHT, where it can detect the touchdown event as usual.

The state machine above only allows a single leg to be in STANCE at any time instance, thus only allowing for trot gaits with an aerial phase. When implemented on Minitaur, we observe a stable trotting pattern as shown in Fig. 21.

The details of the attitude and speed control for the trot gait (both formally and empirically) deserve a more careful examination than is possible within the scope of this paper, such as a formal anchoring of the virtual leg to a monopedal template as was done for RHex in Saranli and Koditschek (2003); we defer this to future work. 